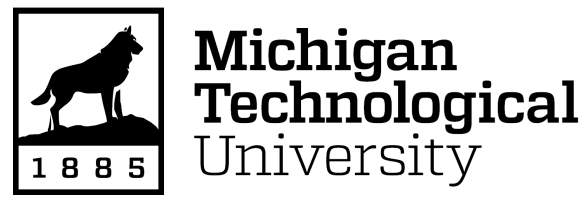

Michigan Technological University Digital Commons @ Michigan Tech

Dissertations, Master's Theses and Master's Reports

2017

\title{
Molecular Modeling of Aerospace Polymer Matrices Including Carbon Nanotube-Enhanced Epoxy
}

Matthew Radue

Michigan Technological University, msradue@mtu.edu

Copyright 2017 Matthew Radue

\section{Recommended Citation}

Radue, Matthew, "Molecular Modeling of Aerospace Polymer Matrices Including Carbon NanotubeEnhanced Epoxy", Open Access Dissertation, Michigan Technological University, 2017.

https://doi.org/10.37099/mtu.dc.etdr/457

Follow this and additional works at: https://digitalcommons.mtu.edu/etdr

Part of the Nanoscience and Nanotechnology Commons, Polymer and Organic Materials Commons, and the Structural Materials Commons 


\title{
MOLECULAR MODELING OF AEROSPACE POLYMER MATRICES INCLUDING CARBON NANOTUBE-ENHANCED EPOXY
}

\author{
By \\ Matthew S. Radue \\ A DISSERTATION \\ Submitted in partial fulfillment of the requirements for the degree of
}

DOCTOR OF PHILOSOPHY

In Mechanical Engineering - Engineering Mechanics

MICHIGAN TECHNOLOGICAL UNIVERSITY

2017

(C) 2017 Matthew S. Radue 

This dissertation has been approved in partial fulfillment of the requirements for the Degree of DOCTOR OF PHILOSOPHY in Mechanical Engineering - Engineering Mechanics.

Department of Mechanical Engineering - Engineering Mechanics

Dissertation Advisor: $\quad$ Dr. Gregory Odegard

Committee Member: Dr. Julia King

Committee Member: Dr. Ranjit Pati

Committee Member: Dr. Gowtham

Department Chair: $\quad$ Dr. William Predebon 



\section{TABLE OF CONTENTS}

LIST OF FIGURES .................................................................................... viii

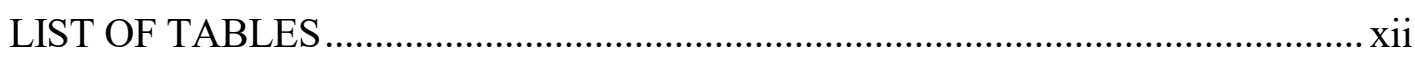

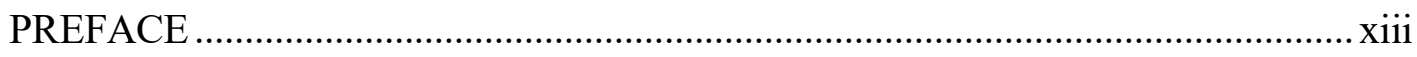

ACKNOWLEDGEMENTS ..............................................................................

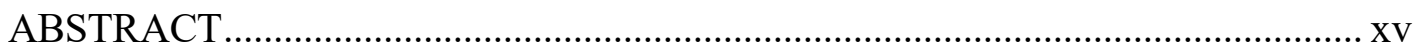

Chapter 1 INTRODUCTION .................................................................... 1

Chapter 2 BACKGROUND .............................................................4 4

2.1 Aerospace Polymer Matrices....................................................................... 4

2.2 Carbon Nanotube / Epoxy Nanocomposites ..................................................... 4

2.3 Molecular Dynamics...........................................................................6

2.4 ReaxFF Reactive Force Field ................................................................... 9

2.5 Modeling the CNT/polymer Interface ........................................................... 11

2.6 MD Comparisons of Various Polymers with CNT ................................... 12

Chapter 3 PURE EPOXY MODELING .......................................14

$3.1 \quad$ Introduction............................................................................................................. 14

3.2 Molecular Modeling ................................................................................... 15

3.3 Specimen Fabrication and Testing ................................................................ 23

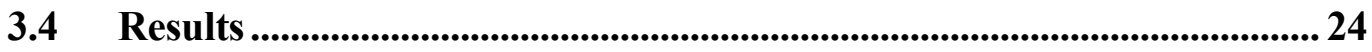

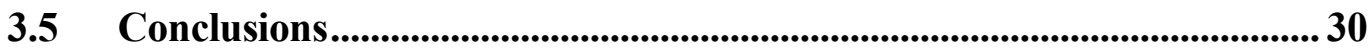

Chapter 4 BMI MODELING ......................................................................31

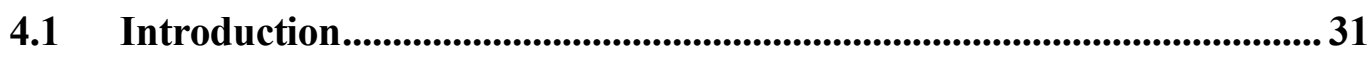

4.1.1 BMI Cure Reactions ......................................................................... 31

4.2 Molecular Modeling …................................................................................................ 34

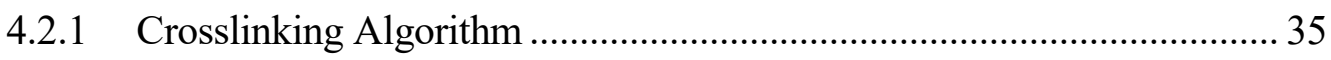

4.2.2 Annealing and Deformation Simulations ........................................... 39

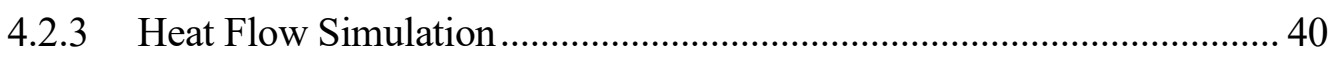

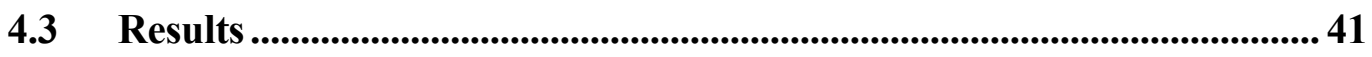

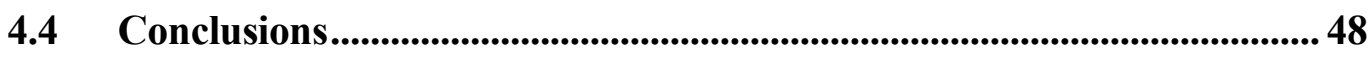

Chapter 5 MULTISCALE CNT/EPOXY MODELING ................50 
$5.1 \quad$ Introduction ...................................................................................................50

$5.2 \quad$ MD Modeling ..................................................................................................52

5.2.1 Model Construction................................................................................

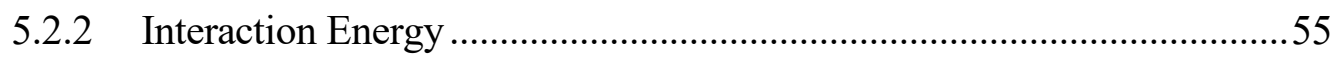

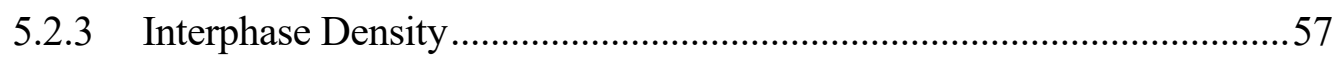

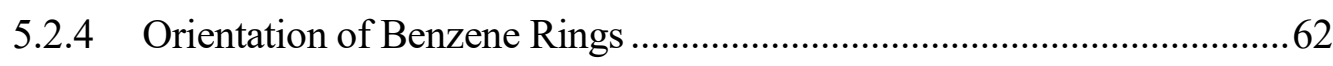

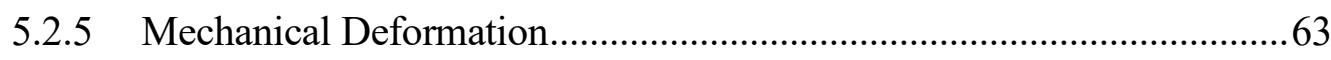

$5.3 \quad$ Micromechanics...................................................................................................65

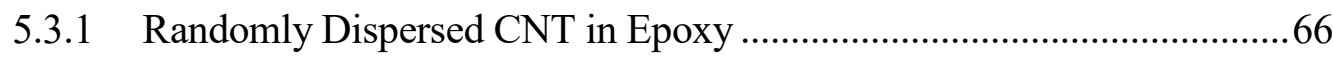

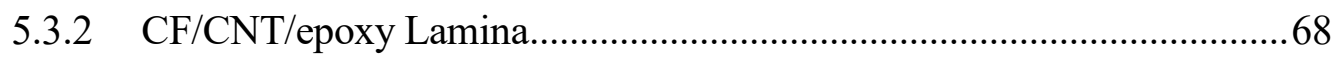

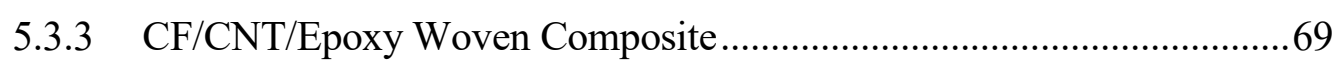

5.4 Multiscale Modeling Predictions ......................................................................70

5.4.1 Randomly Dispersed CNT in Epoxy .................................................... 70

5.4.2 CF/CNT/epoxy Lamina......................................................................

5.4.3 CF/CNT/Epoxy Woven Composite ......................................................... 74

5.5 Conclusions ..........................................................................................................77

\section{Chapter 6 RATE-DEPENDENT YOUNG'S MODULUS........... 78}

6.1 Introduction ...........................................................................................................78

6.2 Standard Linear Solid ................................................................................... 78

6.3 Generalized Maxwell Model ...........................................................................81

6.4 Conclusions ....................................................................................................83

Chapter 7 RECOMMENDATIONS FOR FUTURE WORK..... 84

7.1 Parameterizing ReaxFF for Epoxies..............................................................84

7.2 Thermal Properties of CNT/epoxy ..................................................................84

7.3 Functionalized CNT ...........................................................................................85

7.4 BMI Modeling.........................................................................................................85

Chapter 8 REFERENCES ......................................................... 86

Appendix A Computational Resources ..................................................91

A.1 HPC Specifications...........................................................................................91

A.2 Pure Epoxy ...........................................................................................................91

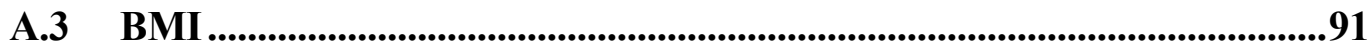

A.4 CNT/epoxy ............................................................................................................92 
Appendix B Sample LAMMPS Scripts ......................................................93

B.1 Mix and Densify Monomers ............................................................................. 93

B.2 Uniaxial Deformation ............................................................................................94

Appendix C Copyright Agreements......................................................997

C.1 Copyright Agreement for Figure 2-1...........................................................97

C.2 Copyright Agreement for Figure 2-2 .......................................................... 103

C.3 Copyright Agreement for Figure 5-12 ......................................................... 104 


\section{LIST OF FIGURES}

Figure 1-1. Carbon Fiber/CNT/Polymer Hybrid Composite

Figure 2-1. Overall CNT/epoxy Young's modulus and tensile stress (normalized by matrix properties) for matrices of varying stiffness [9]. Reproduced with permission of Pergamon. (See Appendix C for copyright agreement)

Figure 2-2. Comparison of harmonic bond and Morse bond [14]. Reproduced with permission of the Mineral Society of America. (See Appendix C for copyright agreement)... 8

Figure 3-1. Epoxy systems modeled and the skeletal structures of the resin and hardener monomers

Figure 3-2. Epoxide-amine crosslinking reactions.

Figure 3-3. Oligomer example with the number of bonded units indicated for each monomeric unit.

Figure 3-4. Representative stress-strain curve from MD simulation.

Figure 3-5. Young's modulus versus strain rate for experimental [62] and predicted results for BFDGE/DETDA. Crosshead speeds shown based on experimental setup by Littell et al. (0.125 in. gauge length). Strain rates typifying the Split Hopkinson Pressure Bar test highlighted in yellow. 26

Figure 3-6. Young's modulus versus MDI for all epoxy models. 27

Figure 3-7. Yield stress versus strain rate for experimental [62] and predicted results for BFDGE/DETDA. Crosshead speeds shown based on experimental setup by Littell et al. (0.125 in. gauge length). Strain rates typifying the Split Hopkinson Pressure Bar test highlighted in yellow. 28

Figure 3-8. Yield stress versus MDI results for all epoxy models. 29

Figure 3-9. Representative transverse strain versus axial strain plot with linear fit. 29

Figure 4-1. Monomer skeletal structures and the proposed cure reactions that were implemented in the present crosslinked Matrimid-5292 model 32

viii 


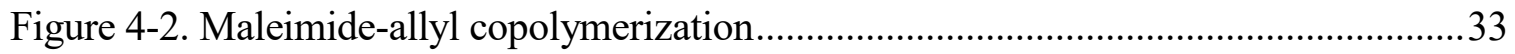

Figure 4-3. Diels-Alder reaction between maleimide and ene adduct. ..................................33

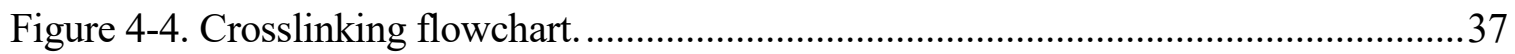

Figure 4-5. (a)-(c) Stacked bar charts indicating the number of cure reactions executed for each cure mechanism in Table 1. Each set of bars shows 4 stages of a particular cure path (3 independent sets). (d)-(f) Number of reactive groups for progressive cure snapshots. ..........38

Figure 4-6. Schematic of heat flow simulation.................................................................. 40

Figure 4-7. Representative density-temperature plot fit with bilinear regression model.......41

Figure 4-8. Representative volume-temperature plot fit with a polynomial regression model and the slope of the volume-temperature curve at $27^{\circ} \mathrm{C}$ (red)..........................................42

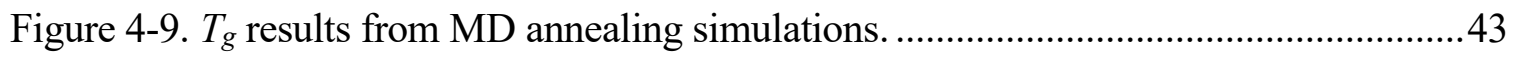

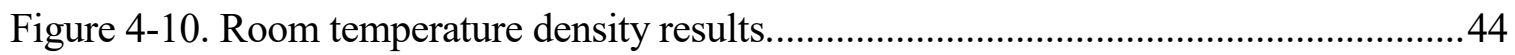

Figure 4-11. MD predictions for the coefficient of linear thermal expansion. .....................45

Figure 4-12. Young's modulus results from MD deformation simulations. .........................46

Figure 4-13. Poisson's Ratios obtained for all MD models............................................46

Figure 4-14. Representative temperature profile. The center region is shown in gray between

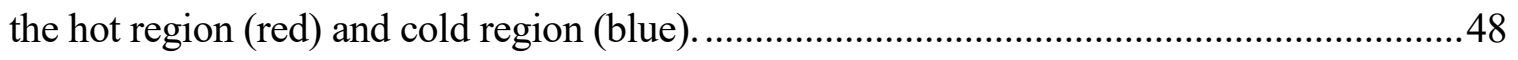

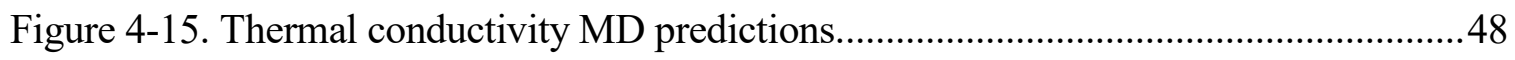

Figure 5-1. Three epoxy systems modeled with CNT and the skeletal structures of each

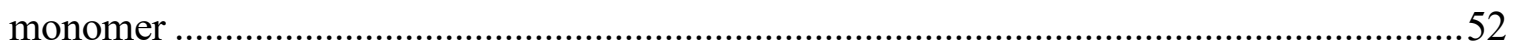

Figure 5-2. Representative CNT (yellow) and epoxy (CPK coloring) model. ......................55

Figure 5-3. Interaction energies for all 15 MD models ....................................................56

Figure 5-4. Contribution to interaction energy by epoxy elements.....................................57

Figure 5-5. Radial distribution profiles for all samples of each epoxy type .........................58 
Figure 5-6. Average radial density profiles for (a) uncured and (b) cured models of each epoxy type. Average taken over five samples................................................................ 59

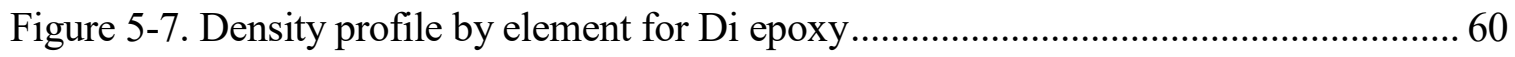

Figure 5-8. Relationship between epoxy density peak and CNT/epoxy interaction energy for all models

Figure 5-9. Orientation of benzene rings with respect to CNT surface for a single CNT/epoxy model

Figure 5-10. Representative stress-strain curve with linear fit 64

Figure 5-11. Multiscale Modeling Scheme. 66

Figure 5-12. 3D RUC for finite CNT aspect ratios

Figure 5-13. MAC/GMC RUC for plain weave fabric [103]. Reproduced with permission of the American Institute of Aeronautics and Astronautics. (See Appendix B for copyright agreement)

Figure 5-14. Normalized modulus for random CNT/epoxy using the Di epoxy (BFGDE/DETDA) as predicted by our multiscale model. Results are shown for various $\mathrm{CNT}$ concentration and $\mathrm{CNT}$ aspect ratios and compared with experimental literature values $[7,104,105,107]$ .71

Figure 5-15. Effective Young's Modulus of random CNT/epoxy for various epoxy types and CNT aspect ratios 72

Figure 5-16. Predicted axial and transverse moduli for CF/CNT/epoxy with the Di epoxy. Various fiber volume fractions and CNT wt $\%$ are shown. CNT is treated as having an infinite aspect ratio. 73

Figure 5-17. Effective transverse modulus for $\mathrm{CF} / \mathrm{CNT} /$ epoxy hybrid laminas for various epoxy types. Volume fraction of CF is 0.60 .

Figure 5-18. Predicted woven composite in-plane Young's modulus for various epoxy types and CNT concentrations. Results are shown for 0.30 overall fiber volume fraction (dashed) and 0.40 volume fraction (solid). 75 
Figure 5-19. Predicted woven composite in-plane shear modulus for various epoxy types and CNT concentrations. Results are shown for 0.30 overall fiber volume fraction (dashed)

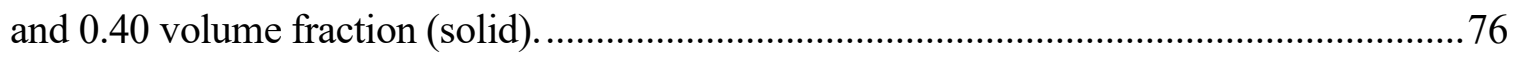

Figure 5-20. Design map for optimizing the in-plane Young's modulus and shear modulus for a woven $\mathrm{CF} / \mathrm{CNT} / \mathrm{epoxy}$ composite with the Di epoxy …......................................... 76

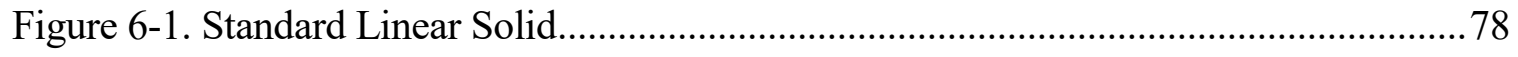

Figure 6-2. Prediction of Young's modulus vs strain rate relationship by the SLS model.

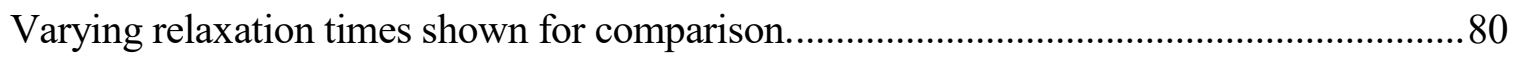

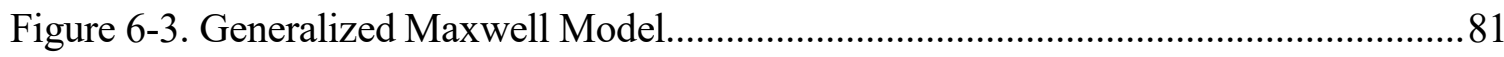

Figure 6-4. Example rate-dependence of Young's modulus given two distinct relaxation modes as predicted by the Generalized Maxwell model. .82 


\section{LIST OF TABLES}

Table 3-1. Modeling details for each epoxy type............................................................. 18

Table 3-2. MDI values and crosslink densities of all models ............................................. 22

Table 3-3. Comparison of mass densities............................................................................ 23

Table 3-4. Comparison of elastic moduli results .................................................................. 25

Table 3-5. Yield point results from simulation ................................................................ 28

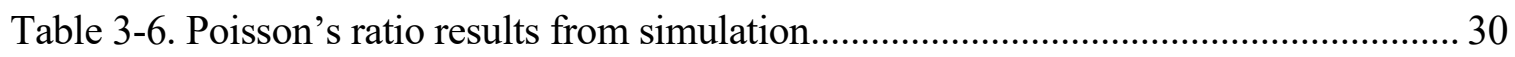

Table 4-1. Reactions incorporated for each mechanism .......................................................... 35

Table 5-1. Details of epoxy matrix for each type …………………………………………....5

Table 5-2. Nanocomposite models..................................................................................... 54

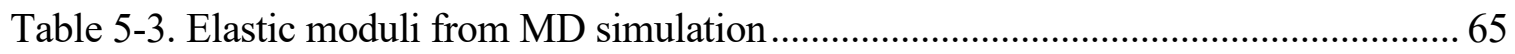

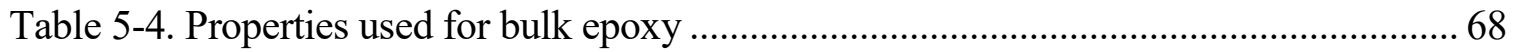

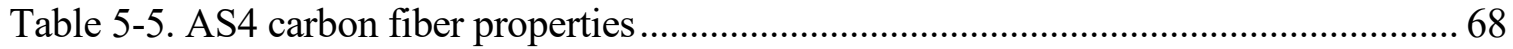




\section{PREFACE}

Dr. Gregory Odegard provided direction for the course of this work as well as reviewed and corrected portions the manuscript. Additional corrections and contributions to the writing of Chapter 3 were given by Dr. Benjamin Jensen. Dr. Danielle Klimek-McDonald and Dr. Julia King supplied the experimental testing in Chapter 3 and the written account thereof. Dr. Vikas Varshney provided important contributions to the writing of Chapter 4. 


\section{ACKNOWLEDGEMENTS}

My sincere thanks to Dr. Gregory Odegard for providing the opportunity to undertake this work and advising me in it. Greg has answered my questions, reviewed my research writing, and celebrated with Val and me on our wedding day!

I greatly appreciate Dr. Ben Jensen's help in learning the fundamentals of Molecular Dynamics. Ben is the one responsible for connecting me with Dr. Odegard and has been an enjoyable officemate and bandmate.

Much thanks goes to those who I had the wonderful opportunity to interact with at AFRL: Dr. Ajit Roy for overseeing my visit and for the tasty jalebi, Dr. Jeff Baur for his inspiring leadership, and especially Dr. Vikas Varshney for deepening my understanding of molecular modeling and teaching me by example how to be a more diligent researcher.

Thanks to my fellow student researchers at Michigan Tech. In particular, Sorayot "Choi" Chinkanjanarot has been a kind friend who has provided good conversations. Will Pisani, congratulations on the new marriage! Hashim Al Mahmud has been pleasure to talk to as well and has been generous to my family. I wish all the group members the best as you continue your research!

For their contributions on the committee, thanks to Dr. Gowtham, Dr. Ranjit Pati, and Dr. Julie King.

I would also like to recognize the pivotal role that my wife, Valerie, has played by supporting and encouraging me over the past five years. She has made many sacrifices so that I could work late on homework or research! Also, I thank my daughter, Lydia, for bringing extra joy into our house.

Above all, thanks and glory to the Lord, my God. May Jesus Christ be central in all I do. God has provided all the wonderful people that have helped me. I sincerely believe that my energy and effort is enabled and sustained by Him.

Unless the Lord builds the house, those who build it labor in vain. 


\begin{abstract}
Carbon fiber (CF) composites are increasingly replacing metals used in major structural parts of aircraft, spacecraft, and automobiles. The current limitations of carbon fiber composites are addressed through computational material design by modeling the salient aerospace matrix materials. Molecular Dynamics (MD) models of epoxies with and without carbon nanotube (CNT) reinforcement and models of pure bismaleimides (BMIs) were developed to elucidate structure-property relationships for improved selection and tailoring of matrices.

The influence of monomer functionality on the mechanical properties of epoxies is studied using the Reax Force Field (ReaxFF). From deformation simulations, the Young's modulus, yield point, and Poisson's ratio are calculated and analyzed. The results demonstrate an increase in stiffness and yield strength with increasing resin functionality. Comparison between the network structures of distinct epoxies is further advanced by the Monomeric Degree Index (MDI). Experimental validation demonstrates the MD results correctly predict the relationship in Young's moduli for all epoxies modeled. Therefore, the ReaxFF is confirmed to be a useful tool for studying the mechanical behavior of epoxies.

While epoxies have been well-studied using MD, there has been no concerted effort to model cured BMI polymers due to the complexity of the network-forming reactions. A novel, adaptable crosslinking framework is developed for implementing 5 distinct cure reactions of Matrimid-5292 (a BMI resin) and investigating the network structure using MD simulations. The influence of different cure reactions and extent of curing are analyzed on the several thermo-mechanical properties such as mass density, glass transition temperature, coefficient of thermal expansion, elastic moduli, and thermal conductivity. The developed crosslinked models correctly predict experimentally observed trends for various properties.

Finally, the epoxies modeled (di-, tri-, and tetra-functional resins) are simulated with embedded CNT to understand how the affinity to nanoparticles affects the mechanical response. Multiscale modeling techniques are then employed to translate the molecular phenomena observed to predict the behavior of realistic composites. The effective stiffness of hybrid composites are predicted for CNT/epoxy composites with randomly oriented CNTs, for CF/CNT/epoxy systems with aligned CFs and randomly oriented CNTs, and for
\end{abstract}


woven $\mathrm{CF} / \mathrm{CNT} /$ epoxy fabric with randomly oriented CNTs. The results indicate that in the CNT/epoxy systems the epoxy type has a significant influence on the elastic properties. For the $\mathrm{CF} / \mathrm{CNT}$ /epoxy hybrid composites, the axial modulus is highly influenced by $\mathrm{CF}$ concentration, while the transverse modulus is primarily affected by the CNT weight fraction. 


\section{Chapter 1 INTRODUCTION}

The carbon fiber composite is a significant achievement in material development which has directly led to advances in lighter and faster aircraft, spacecraft, and automobiles. These composites have excellent stiffness and strength while maintaining a relatively low weight. Additionally, the anisotropic properties allow for parts to be specially engineered to handle expected loads. Carbon fiber composites are increasingly replacing metals used in major structural parts. In regards to aircraft, these composites are being implemented in wings and fuselages. A critical benefit of lightweighting aircraft and other modes of transportation is increased fuel efficiency and consequently lower emissions.

However, there are limitations that hinder the extent to which carbon fiber composites can be deployed. The principal weaknesses are low compressive strength, tendency to delaminate when subjected to impact, and low thermal conductivity. Thus, not only do mechanical deficiencies need to be addressed but also thermal properties since the poor thermal conductivity of polymer matrices leads to premature degradation at high temperature zones of the structure. Improving the thermal conductivity would allow heat to be dissipated faster from hotspots.

In order to enhance the matrix to collectively address these issues, nanoparticles are an appealing option since they have desirable properties and can be mixed into the polymer matrix. This concept combines the advantages of both traditional composites and nanocomposites creating innovative materials known as hybrid composites (see Figure 1-1). Intentionally fabricating a material on multiple scales may provide a breakthrough to obtaining the next generation of carbon fiber composites. Not only could hybrid composites outperform traditional composites in mechanical properties but could potentially provide other valuable properties, such as improved thermal conductivity. For this work, carbon nanotubes (CNTs) are the nanoparticle of interest since they are ultra-strong, ultra-stiff, thermally conductive, and well-researched. The diameters of CNTs are on the order of a nanometer, while half-meter-long CNTs have been reported [1]. The fascinating, superior properties of CNTs make these nanoparticles attractive candidates for emerging 
multifunctional composites. As nanoparticles are embedded into polymer matrices, understanding how the particle interacts with the matrix is critical.

Moreover, further investigation into the thermo-mechanical behavior of polymers allows for more intelligent selection and tailoring of matrices. While the influence of different processing parameters on the final properties has been studied for many of these polymers to an extent, mixing in nanoparticles is known to alter the cure process, and local irregularities are observed in the polymer structure surrounding a nanoparticle. To access the structural alterations in the matrix, a fundamental understanding of how the molecular network behaves is necessary. Developing and elucidating new structure-property relationships in the relevant matrices may then propel composite design on multiple scales.

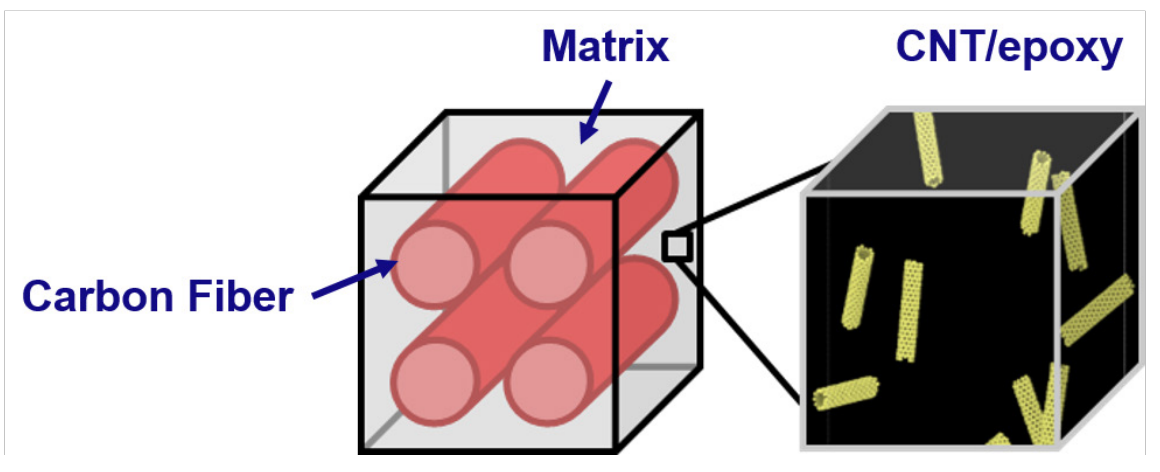

Figure 1-1. Carbon Fiber/CNT/Polymer Hybrid Composite

The overarching goal of this work is to analyze the matrix of carbon fiber hybrid composites in a two-fold manner:

1.) Investigate the structure-property relationships of the matrices independently

2.) Investigate matrix-nanoparticle interactions

A computational approach will be employed to investigate these areas. Both the matrix models and composite models will be validated by comparing the predicted properties with experimental data obtained in this work and data established in the literature. Through material modeling, detailed atomistic information can be gleaned to ascertain the fundamental role of principal molecular characteristics. The effects of different material 
parameters can be explored on the CNT-matrix interaction to determine what matrices will be best suited to pair with CNTs. Additionally, multiscale modeling techniques will be employed to predict the overall hybrid composite properties, thereby evaluating the true significance of CNT-matrix interactions on the macroscale mechanical response. 


\section{Chapter 2 BACKGROUND}

\subsection{Aerospace Polymer Matrices}

The fifth-generation combat aircraft F-35 Lightning II developed by Lockheed is stated to consist of about $35 \%$ carbon fiber composites out of the entire structural weight, and the primary structural composites used in the impressive aircraft are composed of either epoxy or bismaleimide matrices [2, 3]. In general, epoxies are the primary matrix material used for carbon fiber composites for structural aerospace applications. They possess good chemical and electrical resistance and can have high glass transition temperatures as well as excellent adhesion properties. Such resins are commonly applied as adhesives or coatings as well, demonstrating the versatility of epoxies. Bismaleimides (BMIs) offer improved high temperature stability compared to epoxies. BMIs belong to a broader class of materials, polyimides, that includes PMR-15. PMR-15 is notably stronger than BMIs as demonstrated by Aglan et al. [4]. In this study, the failure properties at high temperatures for BMI, PMR15, and PMR-II matrix composites were compared. Flexural tests showed the strength of PMR-15 drop $36 \%$ at $260^{\circ} \mathrm{C}$ when compared to the room temperature strength. Despite the decrease, the PMR-15 (and PMR-II) composites demonstrated superior ultimate strength over BMI. However, the BMI failure properties were shown to be less sensitive to temperature. PMR-15 is also known to be difficult to process due to high curing temperatures and its toxic nature [5], but BMIs are more easily processed in manner similar to epoxies [6].

\subsection{Carbon Nanotube / Epoxy Nanocomposites}

In the extensive research of CNT/polymer nanocomposites, two important themes have emerged: how to prevent agglomeration of CNTs, and how to strengthen the interface between CNT and the matrix. Both are vitally important to obtaining good overall mechanical properties. When it comes to obtaining desirable dispersion, not all CNTs are equally challenging. Gojny et al. investigated the dispersion and mechanical reinforcement 
of various types of CNTs in epoxy: single-walled CNT (SWCNT), double-walled CNT (DWCNT), multi-walled CNT (MWCNT), amine-functionalized DWCNT, and aminefunctionalized MWCNT [7]. Comparing SWCNT, DWCNT, and MWCNTs, we note that an individual SWCNT will have the greatest interfacial area per unit mass than a DWCNT or MWCNT. Therefore, SWCNTs have the greatest potential for improving load transfer. Even so, Gojny et al. demonstrated that SWCNTs are more difficult to disperse than MWCNTs. As a result, SWCNTs did not provide the best enhancement in Young's modulus for both 0.1 and $0.3 \mathrm{wt} \%$ unfunctionalized CNT. Rather, the DWCNTs provided the best improvement, perhaps since DWCNTs possess a good balance of dispersibility and specific surface area for stress transfer.

With the same goal of achieving better nanocomposite mechanical properties, some studies have investigated what kind of epoxy matrices are most favorably influenced by CNTs. Korayem et al. compared two different commercial epoxies used in reinforcing civil structures and found that the more ductile epoxies saw the greater improvement in Young's Modulus for two forms of CNTs, namely CNT powder and CNT masterbatch [8]. Using SEM, it can be observed whether CNTs are mostly fractured or pulled out of the matrix at the fracture surface. Fractured CNTs indicate strong CNT/epoxy adhesion. Similarly, Ci and Bai investigated the effect of the epoxy matrix stiffness by controlling the cure time and the ratio of hardener to resin [9]. Figure 2-1 shows the CNT/epoxy mechanical properties plotted with respect to the matrix modulus. The nanocomposite Young's modulus is normalized by dividing by matrix modulus. Similarly, the normalized tensile stress was obtained by dividing the nanocomposite stress by pure matrix stress at a given amount of strain. As demonstrated in Figure 2-1, soft, ductile epoxies demonstrate the greatest improvement in Young's Modulus for $0.5 \mathrm{wt} \%$ of CNT. For the most compliant matrix, a three-fold improvement in the Young's modulus was observed when adding (unfunctionalized) CNTs. However, virtually no improvement in the mechanical properties occurred for host matrices with moduli above $2.0 \mathrm{GPa}$. This diminished effect of unfunctionalized CNT reinforcement in stiff epoxy matrices has continued to be consistently reported [10-12]. 
Efforts into improving the CNT/polymer interface have led to grafting functional groups onto the CNTs so that the CNTs can be covalently bonded to the matrix. With functionalized CNT, soft matrices are not necessarily most enhanced by CNT. For example, Gude et al. studied the influence of amino-functionalized multi-walled CNT in an epoxy matrix consisting of diglycidyl ether of bispenol A (DGEBA) and 4,4'diaminodiphenylmethane (DDM) [13]. Three different ratios of resin to hardener were studied by adding $0.1 \mathrm{wt} \%$ and $0.25 \mathrm{wt} \%$ of CNT. It was shown that the epoxy with the greatest flexural modulus improved in flexural stiffness by $15 \%$ with $0.25 \mathrm{wt} \% \mathrm{CNT}$. Whereas, the softer epoxies saw little improvement or a decrease in modulus. In contrast to the reports concerning unfunctionalized CNT, the stiffness of the pure epoxy matrix does not appear to be the dominant factor when examining the influence of functionalized CNT.

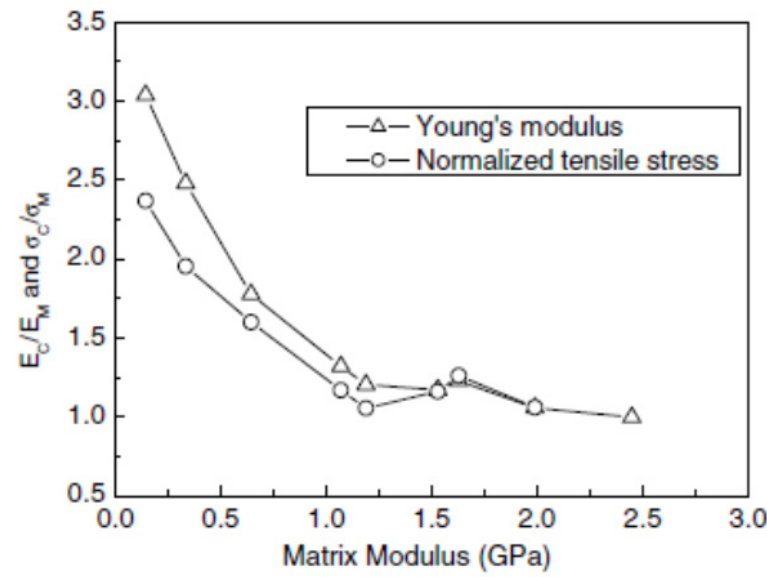

Figure 2-1. Overall CNT/epoxy Young's modulus and tensile stress (normalized by matrix properties) for matrices of varying stiffness [9]. Reproduced with permission of Pergamon. (See Appendix C for copyright agreement)

\subsection{Molecular Dynamics}

Molecular Dynamics (MD) has become an important tool for studying the behavior of materials and predicting thermo-physical and mechanical properties. This technique allows for the defining features of the molecular structure to be directly linked to observed macroscopic properties. Material parameters can easily be explored to suggest what would 
be ideal and thereby provide a target for researchers in the laboratory. Additionally, conceptual materials can be judged worthy of pursuit. A computational approach for analyzing materials is relatively quick and inexpensive and can provide important details about the material behavior on the molecular level.

Using classical mechanics, MD software simulates the trajectories of individual atoms are over a short period of time. The user sets the initial positions, velocities, and masses of each atom and sets the equations that govern interatomic forces. Typically, a type of Verlet algorithm is then used to advance the positions and update the velocities of all atoms for a tiny step ahead in time $(0.1-1 \mathrm{ps})$ and alternately recalculate the interatomic forces.

The set of equations defining interatomic forces is known as a force field. To demonstrate a few key concepts about a MD force field, consider two bonded atoms. One potential energy form that is frequently used is the harmonic bond, given by the following equation:

$$
U=K\left(r-r_{0}\right)^{2}
$$

Here, $U$ is the potential energy, $r$ is the distance between atoms, and $K$ and $r_{0}$ are parameters. $K$ refers the stiffness of the bond, and $r_{0}$ is the bond equilibrium distance. The $K$ and $r_{0}$ parameters are determined via experimental techniques and quantum calculations in the development of the force field. Figure 2-2 shows a harmonic bond potential for a $\mathrm{C}-\mathrm{H}$ bond. The harmonic bond is a simple functional form that mimics the behavior of a spring which suffices when all bonds are expected to remain close to the equilibrium distance. However, for greatly stretched bonds, the harmonic bond equation results in a large restoring force, which is unrealistic. Thus, this functional form cannot accurately model bond dissociation.

However, other bond potentials attempt to capture the interatomic force approaching zero as the bond is increasingly stretched, such as the Morse potential given by the following functional form:

$$
U=D_{0}\left[1-\exp \left(-\alpha\left(r-r_{0}\right)\right)\right]^{2}
$$


The Morse potential requires three parameters for every bond, namely $D_{0}, \alpha$, and $r_{0}$. Figure 2-2 shows a Morse bond in comparison with a harmonic bond for C-H. In addition to bond potentials, there are a number of other contributions that are summed together to express the total potential energy. These additional terms often include van der Waals interactions, electrostatic interactions, angles, and dihedrals. Furthermore, each term also has a functional form and numerous atom-dependent parameters.

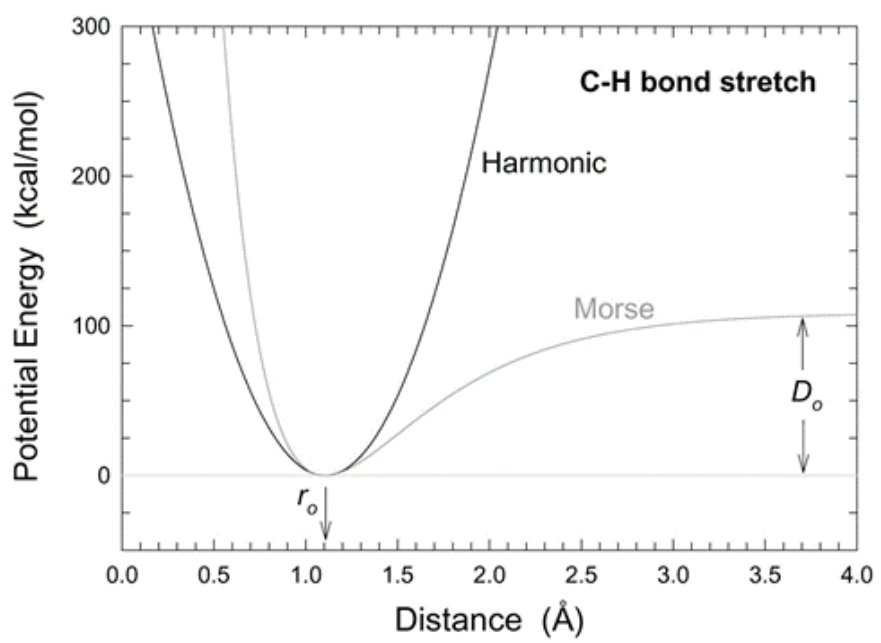

Figure 2-2. Comparison of harmonic bond and Morse bond [14]. Reproduced with permission of the Mineral Society of America. (See Appendix C for copyright agreement)

While a phenomenon may consist of important events that occur on many different length scales, MD is equipped to work on the nanoscale alone. Thus, it is important to focus on an event that can be described with roughly 100,000 atoms and occurring over several tens of nanoseconds. Beyond this, computations often become too cumbersome to be feasible. The limitation in system size and time is partially overcome by employing techniques for scaling up the results. Micromechanics is one such method that is used to bridge the chasm between nanoscale and macroscale and will later be applied in this work. 


\subsection{ReaxFF Reactive Force Field}

Using a harmonic bond or Morse bond potential requires that every bond is explicitly set by the user. Usually these pre-set bonds do not change throughout the simulation. However, a condition may be enforced to break a bond if it exceeds some critical length. Even so, this does not allow an $\mathrm{sp} 3$ carbon to become sp2 during a simulation without predetermined, direct intervention. When modeling the failure of a material under large strain, it is important for the force field to autonomously predict changes in bond configuration, which cannot be accomplished by a fixed-bond force field. However, the Reax Force Field (ReaxFF) assigns a bond order (1 for single bond, 2 for double bond, etc.) for all relevant pairs of atoms based on the interatomic distance. The corresponding interatomic forces are determined based on the bond order. Bond orders are updated throughout a simulation allowing for the formation and breaking of bonds. All parameters used by the ReaxFF are characterized a priori to reproduce results from quantum mechanics computations for select, small molecular systems.

The original ReaxFF parameter set was published in 2001 and parameterized for small hydrocarbons [15]. Like other force fields, the total potential energy is the sum of many energy terms. In the original ReaxFF, the following energy terms were present: bond, overcoordination, under-coordination, valence angle, double-bond valence angle penalty, torsion, conjugation, van der Waals, and Coulomb energies. Most of these energy terms (besides van der Waals and Coulomb energies) are explicitly calculated from the atomic bond order. The bond, angle, and torsion energies describe analogous bond, angle, and torsion energies seen in other force fields with the difference being the bond-orderdependence of the ReaxFF terms. The over-coordination and under-coordination are both energy penalties imposed when the total bond order of an atom is above or below the expected ideal number, such as 4 for carbon atoms. The double-bond valence angle penalty is for a specific case where two $\mathrm{C}=\mathrm{C}$ bonds form an angle, and the conjugation energy captures the stability of alternating single and double bonds observed, for example, in benzene. The original ReaxFF parameter set was validated by comparing the heats of formation, equilibrium bond distances, and equilibrium angles for numerous hydrocarbons 
to experimental literature values. Further validation was performed by comparing the ReaxFF bond dissociation curves and rotational energy barriers to quantum mechanics calculations.

Odegard et al. demonstrated that ReaxFF provides reliable results for determining elastic moduli and the yield point of epoxies [16]. It is also demonstrated that extrapolating from experimentally obtained strain-dependent mechanical properties can explain results obtained from MD simulation despite the disparity of strain rates between MD and experiment. Subramanian et al. captured the plastic deformation of CNT/epoxy MD models with ReaxFF [17]. The selected epoxy consisted of bisphenol F diglycidyl ether (BFDGE) and diethylene triamine (DETA). A stochastic multiscale model was developed by characterizing the variation of effective elastic modulus and building a Representative Volume Element (RVE) consisting of cells of varying properties. The overall stiffness of the RVE was then calculated using the Finite Element Method. The Singh et al. parameter set [18] was chosen to model CNT/epoxy based on the bond dissociation energy of the C-H bond in hydrogen-terminated CNT.

Besides analyzing the behavior under large strains, another area where ReaxFF has been utilized for studying epoxies is to analyze thermal decomposition. Diao et al. used ReaxFF to investigate the thermal decomposition of an epoxy resin used in printed circuit boards [19]. Various heating rates and final heating temperatures were simulated. While $\mathrm{CH}_{2} \mathrm{O}$ was the initial product for all simulations, for higher temperature heating it was shown that $\mathrm{H}_{2}$ superseded $\mathrm{CH}_{2} \mathrm{O}$ becoming the main product. Furthermore, the reaction pathways to obtaining these products were able to be thoroughly analyzed using ReaxFF. Similarly, Zhang et al. studied the decomposition of the same epoxy resin comparing the effects of microwave heating to traditional heating [20]. Microwave heating was simulated by using the NVE ensemble and applying an oscillating electric field to the entire system. In this case, the Mattsson et al. parameter set was applied [21]. 


\subsection{Modeling the CNT/polymer Interface}

Essential to the performance of a composite is its ability to transfer load from the matrix to the filler, but the question of how efficient the load can be transferred in a nanocomposite is not easy to determine. Wetting, spectroscopy, and force microscropy techniques can be used to assess the strength of an interface [22]. For example, an experimental method that has been successfully demonstrated to quantify the interface between a CNT and polymer involves placing a CNT on an atomic force microscopy (AFM) tip, lowering the CNT into liquid polymer, cooling the polymer to solidify it, and then finally extracting the CNT. The AFM tip captures the force it takes to extract the CNT out of the polymer. In addition to these experimental techniques, atomistic models have proven useful in characterizing the interface. Useful modeling methods include MD pull-out simulations, calculating the interaction energy using Density Functional Theory (DFT) or MD, and modeling the structure of the interphase region.

Simulations of a CNT being extracted from an epoxy substrate have been modeled in order to directly parallel similar experimental tests $[23,24]$. The models can be used to predict the interfacial shear force interfacial shear stress, and interfacial fracture energy. Depending on the nanoparticle and polymer used, the modeling results may be directly compared with experimental results. However, successfully executing an experimental pullout test may prove too difficult for some nanofillers, such as graphene [25].

Another valuable metric for characterizing the interface is the interaction energy which can be obtained from both DFT and MD calculations. The interaction energy is given by the following equation:

$$
E_{\text {interaction }}=E_{A, B}-E_{A}-E_{B}
$$

$E_{A}$ and $E_{B}$ are the potential energies of component $\mathrm{A}$ and component $\mathrm{B}$ modeled separately, and $E_{A, B}$ is the resulting potential energy when component $\mathrm{A}$ and $\mathrm{B}$ are modeled together and interacting. For example, component A may be a CNT, and component B may be a single polymer chain. Favorable interaction is indicated by a negative value, and a more 
negative interaction energy means better interaction. This technique was employed by Ahangari et al. in order to observe the effect of CNT defects on the interaction with epoxy [26]. Tight-binding DFT was used to calculate the interaction energy, and three different defects were modeled. It is also claimed that there is better binding of epoxy with zigzag CNTs than armchair CNTs.

Also, MD modeling can be utilized to observe the nature of the interphase region. As demonstrated by Hadden et al. [27], the crystallinity of the interphase region in a graphite/epoxy composite can be illustrated by calculating the mass density of the epoxy in sections. The $10 \AA$ interphase region observed experimentally was seen in the models as well, regardless of the percentage of crosslinking in the matrix. The von Mises stress and potential energy at the interphase was also analyzed.

\subsection{Comparisons of Various Polymers with CNT}

In considering what types of epoxies are best suited to be paired with CNTs, it is also worthwhile to look to modeling efforts comparing the interaction of various polymers with CNT. Numerous studies have approached this task by performing interaction energy calculations. While these studies do not involve modeling epoxies, the results may be helpful in determining what molecular features allow for good adhesion to CNTs.

Yang et al. used MD interaction energy calculations for CNTs with four different polymers all containing aromatic rings [28]. Two polymers had aromatic rings on the polymer's side chains; whereas, the other two polymers have aromatic rings in the backbone of the polymer. It was demonstrated that the interaction between CNTs and polymers improves as the CNT diameter increases. Additionally, every aromatic ring in the polymer was fit by a plane, and the nearest hexagon on the CNT was likewise fit by a plane. The angle between these two planes was calculated in order to see how the benzene rings in the polymer align themselves with respect to the surface of the CNT. From this it was shown that polymers with aromatic rings on the side chains tended to align their rings perpendicular to the CNT; whereas, the polymers with aromatic rings along the backbone where able to align their rings parallel to the nanotube surface. The polymers that could align its benzene 
rings parallel to the CNT demonstrated significantly better interaction. It was concluded by the authors that this was due to better $\pi-\pi$ stacking. Additional studies have shown that the presence of aromatic rings results in good interaction with CNT [29]. Thus, the structure of the monomers can have a significant effect on the interaction energy. Zaminpayma et al. also showed the same trend of interaction energy versus CNT diameter for the binding of CNT with four different polymers [30]. ReaxFF was used to study this and the influence of temperature and CNT chirality on the interaction. It was found that armchair CNTs provided the best interaction with the polymers considered. Also, the authors suggest that the presence of sulfur was the deciding factor in the polymer with the best interaction with CNT. 


\section{Chapter 3 PURE EPOXY MODELING}

\subsection{Introduction}

Epoxies are highly processable materials with excellent chemical and electrical resistance, high glass transition temperatures, and excellent adhesion properties relative to other polymer systems. When reinforced with high-strength fibers, epoxy-matrix composites provide great stiffness and strength while maintaining a relatively low weight. Thus, epoxymatrix composites are increasingly being investigated and implemented in next-generation aircraft to replace metals used in major structural parts, such as wings and fuselages. The result is increased fuel efficiency and consequently lower emissions. Furthermore, in seeking improvement of the thermal conductivity and mechanical properties of epoxies, efforts have extended to incorporating various nanofillers. As epoxies continue to be valuable in fiber composites and nanocomposites, understanding the molecular behavior of epoxies remains critical. Developing and elucidating structure-property relationships in epoxies themselves may propel composite design on multiple scales. One such important structural feature to consider is the functionality of each monomer, that is, the number of monomers that can potentially be covalently bonded to a given monomer.

Experimental studies that have compared the mechanical properties of epoxies while varying the functionality of either the resin or hardener have shown mixed results. In a study from Becker et al., the pure epoxy results suggest an increase in flexural modulus with an increase in the functionality of the resin [31]. However, other studies do not yield a lucid link between the modulus or yield strength and the monomer functionality [32-34]. Differences in crosslink density or differences in other features of the monomer structure may obscure the effect of functionality. Early efforts to understand the mechanical response of polymers by rubber elasticity theory focused attention on the role of functionality. Crawford and Lesser later applied this theory to epoxies showing how the rubbery moduli and the compressive yield stress relate to the functionality and the molecular weight between crosslinks [35]. 
Molecular Dynamics (MD) can be used to analyze epoxies on the nanometer lengthscale in order to understand the influence of molecular structure on thermo-mechanical properties. Using MD the mechanical response of epoxies has been predicted with respect to system size [36], mass ratio of resin to hardener molecules [37], strain rate [16, 38, 39], moisture content [40], and temperature [38-41]. Additionally, many MD studies have also considered the effect of crosslink density on the mechanical properties of epoxies, and it is clear that the stiffness increases with an increasing number of crosslinks [36, 39-45]. However, fewer MD studies have tackled the role of functionality on the stress-strain response [46-48]. Among these studies, Tsige et al. implemented a coarse-grained model with two potential terms and showed the yield strength, ultimate strength, and strain at failure to depend on functionality [48]. Particularly, the yield strength was shown to increase with increasing functionality. Li et al. undertook a more detailed MD approach by modeling a specific epoxy resin with various hardeners containing either two or three amine groups [46]. However, no all-atom modeling study has thoroughly compared the elastic properties and yielding characteristics for different epoxies having at least three unique combinations of monomer functionalities.

The goal of this chapter is to predict the mechanical properties of a di-functional, trifunctional, and tetra-functional resin epoxy using MD. The deformation of multiple crosslinked samples of each epoxy type were simulated using a reactive force field. The corresponding Young's modulus, Poisson's ratio, and yield point were predicted and compared. Additionally, the effect of network characteristics on these properties were analyzed. For model validation, the predicted mechanical properties were compared to experimentally-obtained values obtained herein, as well as literature values.

\subsection{Molecular Modeling}

To simulate the levels of strain associated with the bulk failure of epoxies, it is expected that covalent bonds will be stretched beyond the distances that traditional fixed-bond force fields are characterized for. Additionally, most fixed-bond MD force fields are not developed to predict changes in bond configuration. ReaxFF can capture large magnitudes 
of stretching and simulate the creation and scission of covalent bonds by assigning a bond order ( 1 for single bond, 2 for double bond, etc.) for all relevant pairs of atoms based on the interatomic distance. The corresponding interatomic forces are determined based on the bond order, and the bond orders are updated throughout a simulation allowing for the creation and breaking of bonds. All parameters used by ReaxFF are optimized a priori to reproduce results from quantum mechanics computations for select, small molecular systems.

In this work, the reactive force field ReaxFF [15] with the low-gradient corrected parameters of Liu et al. [49] were selected and implemented using LAMMPS [50, 51]. The development of the Liu et al. parameter set traces back to the original ReaxFF parameter set by van Duin et al. [15]. The original ReaxFF parameter set was parameterized to reproduce experimental heats of formation and bond and angle data for small hydrocarbons. Additionally, hydrocarbon bond dissociation curves and rotational energy barriers from quantum mechanics calculations were included for determining the parameters. Strachan et $a l$. extended the original ReaxFF parameter set to include nitrogen and oxygen for studying the thermal decomposition of $\operatorname{RDX}[52,53]$. The training set was expanded to include DFT bond dissociation, angle bending, and torsion rotation energies for a variety of CHNO molecules. The Strachan et al. parameter set served as the basis of the Liu et al. parameter set. Liu et al. added a new ReaxFF energy term to improve the accuracy of long-range van der Vaals interactions for molecular solids [49]. The correction parameters were designed to reproduce the experimental heats of formation and densities of the crystal structures of graphite, polyethene, carbon dioxide, solid nitrogen, and energetic materials. The Liu et al. parameter set was selected since it contains the required elements for modeling epoxide and amine groups, demonstrates improved van der Waals interactions for solids, and has been shown to provide reliable results for determining elastic moduli and the yield point of epoxies [16]. 


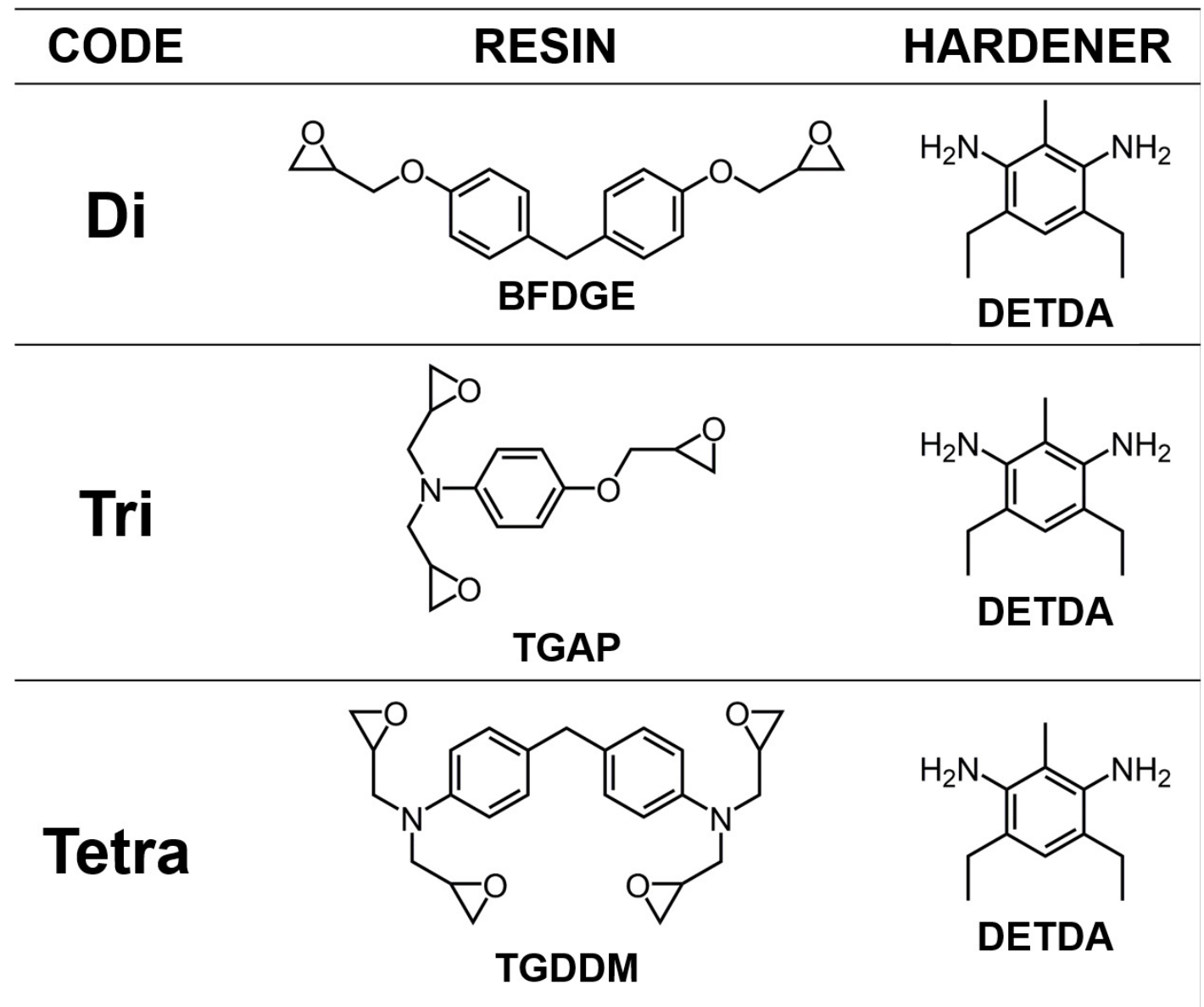

Figure 3-1. Epoxy systems modeled and the skeletal structures of the resin and hardener monomers

The resin molecules chosen were bisphenol F diglycidyl ether (BFDGE, EPON 862), triglycidyl para-amino phenol (TGAP, Araldite MY 0510), and tetra-glycidyl-4,4'diaminodiphenylmethane (TGDDM, Araldite MY 721). One hardener was used, namely, diethyltoluenediamine (DETDA). Using these resins and hardener, three different epoxy systems were composed: BFDGE/DETDA, TGAP/DETDA, and TGDDM/DETDA. The epoxy systems modeled and the molecular structures of the monomers are shown in Figure 3-1. The functionality of the resin monomers is determined by the number of epoxide groups. The number of epoxide groups of BFDGE, TGAP, and TGDDM are 2, 3, and 4 respectively, which is the key distinguishing factor between each epoxy. Thus, the names Di, Tri, and Tetra refer to the epoxies BFDGE/DETDA, TGAP/DETDA, and TGDDM/DETDA, respectively, as indicated in Figure 3-1. Each amine group can react with two epoxide groups during crosslinking. Thus, the ratio of resin monomers to hardener 
monomers was chosen so that exactly two epoxide groups were present for every amine group. For the di-, tri-, and tetra-functional resin epoxies, the ratio of resin to hardener molecules was $2: 1,4: 3$, and 1:1 respectively. Table $3-1$ gives the resin functionality $f_{r}$, the number of resin and hardener molecules, and the total number of atoms for each epoxy model.

Table 3-1. Modeling details for each epoxy type

\begin{tabular}{|c|c|c|c|c|c|}
\hline EPOXY & $\boldsymbol{f}_{\boldsymbol{r}}$ & $\begin{array}{c}\text { NO. OF } \\
\text { RESINS }\end{array}$ & $\begin{array}{c}\text { NO. OF } \\
\text { HARDENERS }\end{array}$ & $\begin{array}{c}\text { RESIN TO } \\
\text { HARDENER RATIO }\end{array}$ & $\begin{array}{c}\text { TOTAL NO. } \\
\text { OF ATOMS }\end{array}$ \\
\hline $\mathrm{Di}$ & 2 & 90 & 45 & $2: 1$ & 5265 \\
$\mathrm{Tri}$ & 3 & 84 & 63 & $4: 3$ & 5229 \\
Tetra & 4 & 57 & 57 & $1: 1$ & 5244 \\
\hline
\end{tabular}

To perform the epoxide-amine crosslinking reactions, high-energy bonds were created as an initial step. Since OPLS is a fixed-bond force field, all other bonds will remain intact when large energy spikes are introduced in the system. It is expected that creating highenergy bonds in ReaxFF would result in unintended dissociation of neighboring bonds. To ensure that the epoxy molecular structure is not inadvertently damaged upon network formation, all epoxy models were initially built using the OPLS all-atom force field [54].

First, the monomers were arrayed sparsely in a periodic simulation box. The initial mass densities were in the range of $0.09-0.10 \mathrm{~g} / \mathrm{cm}^{3}$. A fixed-volume simulation of $100 \mathrm{ps}$ was performed to allow the monomers to mix, in which the temperature was gradually ramped down from $600 \mathrm{~K}$ to $300 \mathrm{~K}$. For each epoxy system, five independent uncrosslinked samples were generated. To ensure the uniqueness of each sample, different initial velocities were assigned for each sample prior to the mixing process. Following these mixing simulations, the simulation boxes were slowly compressed to densities of $1.21 \mathrm{~g} / \mathrm{cm}^{3}$. These simulations occurred at $300 \mathrm{~K}$ for a total of $4 \mathrm{~ns}$, and 20 molecular minimizations were performed at regular intervals during the process. 

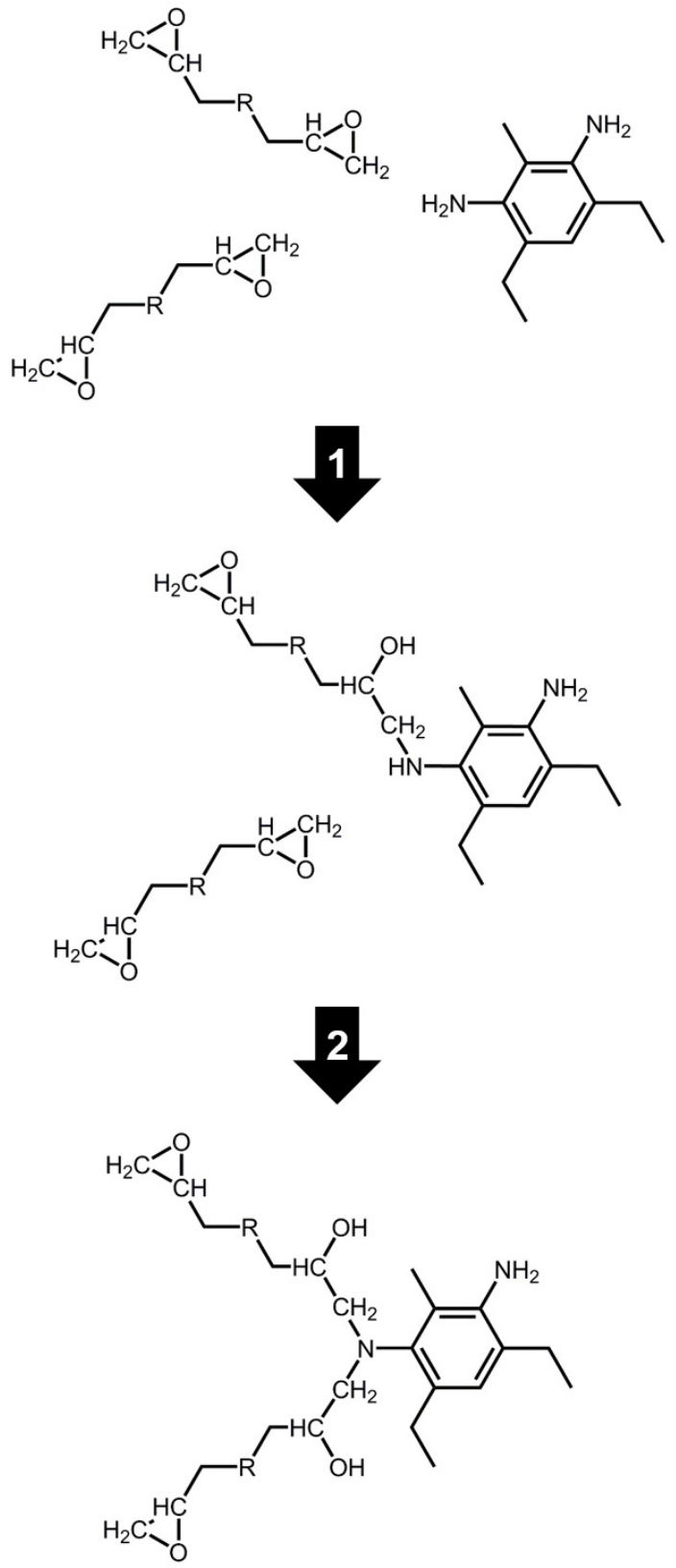

Figure 3-2. Epoxide-amine crosslinking reactions.

The monomers were crosslinked by executing the epoxide-amine reactions shown in Figure 3-2. Reaction 1 shows one epoxide group and a primary amine reacting to open the epoxide ring and form a hydroxyl group and a secondary amine. Moreover, the secondary amine can proceed to react with another epoxide group (Reaction 2) forming a tertiary amine and another hydroxyl group. These reactions were accomplished using a series of 
LAMMPS commands including 'fix bond/create' and 'fix bond/break'. An eligible reaction occurred when a carbon in an epoxide group and a nitrogen in an amine group were at a distance of 7 angstroms or less. Each eligible reaction was randomly confirmed to react. The approved reactions proceeded by applying a harmonic bond potential between the $\mathrm{C}$ and $\mathrm{N}$. The $\mathrm{C}-\mathrm{O}$ epoxide bond and one $\mathrm{N}-\mathrm{H}$ amine bond were subsequently broken, and a new hydroxyl group was created. In order to help bring newly created bonds close to their equilibrium distance, the Langevin thermostat was used [55, 56] along with the 'fix nve/limit' command which places an upper bound on the distance an atom can move in a single time step. The crosslinking process was carried out for up to $1 \mathrm{~ns}$. For some monomer samples, multiple crosslinked models were created by executing the crosslinking process for varying durations. Upon crosslinking, no angles or dihedrals were added since the models would later be simulated with ReaxFF, which independently accounts for the angles and dihedrals.

We define the monomeric degree index (MDI) as the average number of monomeric units that are covalently bonded to a given monomeric unit. This average is taken over all monomeric units in the MD model. A simple oligomer example is shown in Figure 3-3 where the number of bonded units is denoted. Averaging over all resins and hardeners, the MDI is about 1.67 , that is, on average a monomer unit is bonded to 1.67 units. The crosslink density can also be determined for this example. The crosslink density can be defined as the number of reactions performed during crosslinking divided by the maximum number of theoretically possible reactions. In this example, 5 bonds are formed out of a total of 8 possible bonds giving a crosslink density of 0.625 .

Two purposes are served by introducing the MDI. First, the MDI elucidates the nature of network junctions in the epoxy models. The usual measure of functionality describes the potential number of monomeric units that can be bonded to a given monomeric unit. However, during the MD crosslinking procedure, not all reactive sites will engage in a reaction; e.g., a monomer that is capable of covalently bonding to four monomers may only bond to two or three monomers. Thus, the MDI is chosen to describe the network structure in light of incomplete bonding. Secondly, the MDI serves as an alternative to the crosslink density to measure the degree of cure. The crosslink density is defined using the maximum 
number of theoretically possible reactions, which differs between the epoxies studied here. Because the MDI is independent of the number of theoretically possible reactions, it is more suitable than the crosslink density for comparing dissimilar epoxies.
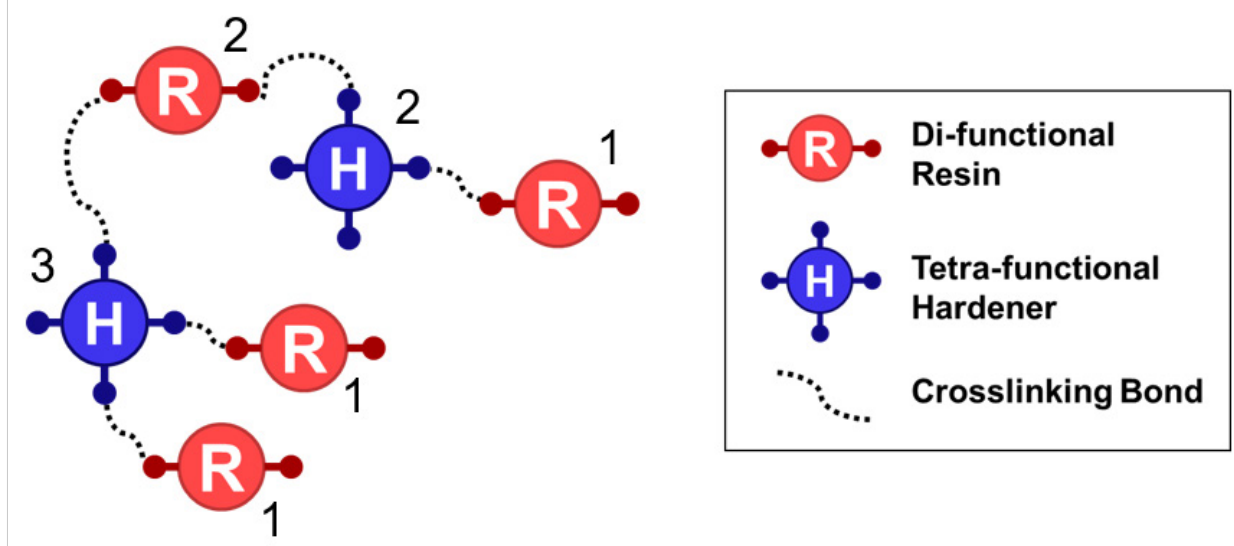

Figure 3-3. Oligomer example with the number of bonded units indicated for each monomeric unit

The MDI values and crosslink densities of all models are shown in Table 3-2. Five models for each epoxy type are highlighted as characteristically crosslinked. A range of experimental crosslink densities of $60 \%-95 \%$ is typically observed for epoxies [57-60]. The characteristically crosslinked models possess crosslink densities of about $70 \%-85 \%$, placing these models in the middle of the experiment range. The five characteristically crosslinked models were selected for predicting the mechanical properties of each epoxy. The remaining low crosslinked models were developed as supplemental models to establish the MDIproperty relationships described below.

As expected, the Di model is not capable of attaining high MDIs relative to the other epoxies, and the Tetra model produces the greatest MDI values. For each epoxy type, a range of MDIs were generated from about 1.5 to the maximum value. Fewer Di models than Tri or Tetra were required to span this range. Table 3-2 also shows that the characteristically crosslinked Tri models obtained the highest average crosslink density. The smaller size of TGAP (having one benzene ring instead of two as with BFDGE and TGDDM) is the suspected cause of the higher crosslinking percent. 
Table 3-2. MDI values and crosslink densities of all models

\begin{tabular}{|c|c|c|c|c|}
\hline \multirow[t]{8}{*}{ EPOXY } & $\begin{array}{c}\text { CURE } \\
\text { CATEGORY }\end{array}$ & $\begin{array}{c}\text { MODEL } \\
\text { NO. }\end{array}$ & MDI & $\begin{array}{c}\text { CROSSLINK } \\
\text { DENSITY }\end{array}$ \\
\hline & \multirow{5}{*}{$\begin{array}{l}\text { Characteristic } \\
\text { Crosslinking }\end{array}$} & 1 & 2.27 & 0.85 \\
\hline & & 2 & 2.24 & 0.84 \\
\hline & & 3 & 2.19 & 0.82 \\
\hline & & 4 & 2.05 & 0.77 \\
\hline & & 5 & 1.89 & 0.71 \\
\hline & \multirow{2}{*}{$\begin{array}{c}\text { Low } \\
\text { Crosslinking }\end{array}$} & 6 & 1.54 & 0.58 \\
\hline & & 7 & 1.45 & 0.54 \\
\hline \multirow{11}{*}{111} & \multirow{5}{*}{$\begin{array}{l}\text { Characteristic } \\
\text { Crosslinking }\end{array}$} & 1 & 2.91 & 0.85 \\
\hline & & 2 & 2.88 & 0.84 \\
\hline & & 3 & 2.78 & 0.81 \\
\hline & & 4 & 2.78 & 0.81 \\
\hline & & 5 & 2.67 & 0.78 \\
\hline & \multirow{6}{*}{$\begin{array}{c}\text { Low } \\
\text { Crosslinking }\end{array}$} & 6 & 2.52 & 0.73 \\
\hline & & 7 & 2.48 & 0.72 \\
\hline & & 8 & 1.93 & 0.56 \\
\hline & & 9 & 1.88 & 0.55 \\
\hline & & 10 & 1.56 & 0.46 \\
\hline & & 11 & 1.48 & 0.43 \\
\hline \multirow{11}{*}{ etra } & \multirow{5}{*}{$\begin{array}{l}\text { Characteristic } \\
\text { Crosslinking }\end{array}$} & 1 & 3.20 & 0.80 \\
\hline & & 2 & 3.16 & 0.79 \\
\hline & & 3 & 3.12 & 0.78 \\
\hline & & 4 & 3.00 & 0.75 \\
\hline & & 5 & 2.88 & 0.72 \\
\hline & \multirow{6}{*}{$\begin{array}{c}\text { Low } \\
\text { Crosslinking }\end{array}$} & 6 & 2.62 & 0.65 \\
\hline & & 7 & 2.53 & 0.63 \\
\hline & & 8 & 2.33 & 0.58 \\
\hline & & 9 & 2.21 & 0.55 \\
\hline & & 10 & 1.90 & 0.47 \\
\hline & & 11 & 1.76 & 0.44 \\
\hline
\end{tabular}

The crosslinked models were switched from OPLS to ReaxFF with the parameter set containing low gradient corrections developed by Liu et al. [49]. The above-described procedure of neglecting the angle and dihedral parameters associated with the newly formed crosslinks caused some hydroxyl groups to dissociate in ReaxFF. However, this was rectified by a 100 ps simulation where all atoms were frozen except the hydroxyl group hydrogens, which were limited in kinetic energy by the 'temp/rescale' and 'fix viscous' commands. This allowed ReaxFF to correct the C-O-H angles. Afterwards, all atoms were gradually brought into motion by ramping the temperature from $1 \mathrm{~K}$ to $300 \mathrm{~K}$ over $100 \mathrm{ps}$.

Equilibration occurred over $1.5 \mathrm{~ns}$ at $300 \mathrm{~K}$. Using the NPT ensemble, the Nose-Hoover barostat was set to maintain 1 atm of pressure on all sides of the simulation box. A timestep of 0.1 fs was used. Table 3-3 provides the average mass density after equilibration for the 
characteristically crosslinked models. All uncertainty measures used in this paper indicate the standard deviation. The model densities are compared with experimental measurements in Table 3-3 showing good agreement.

Table 3-3. Comparison of mass densities

\begin{tabular}{|c|c|c|}
\hline EPOXY & $\begin{array}{c}\text { MODEL DENSITY } \\
\mathbf{g} / \mathbf{c m}^{\mathbf{3}}\end{array}$ & $\begin{array}{c}\text { EXPERIMENTAL DENSITY } \\
\mathbf{g} / \mathbf{c m}^{\mathbf{3}}\end{array}$ \\
\hline $\mathrm{Di}$ & $1.222 \pm 0.005$ & $1.20[61]$ \\
Tri & $1.235 \pm 0.011$ & $1.24[31]$ \\
Tetra & $1.221 \pm 0.002$ & $1.20[31]$ \\
\hline
\end{tabular}

After establishing the equilibrated models, each model was subjected to three uniaxial tensile deformation simulations: tension in the $\mathrm{x}-\mathrm{,}, \mathrm{y}-$, and $\mathrm{z}$ - directions. Deformations were performed every timestep amounting to $20 \%$ engineering strain over $1 \mathrm{~ns}$, resulting in a strain rate of $2 \times 10^{8} \mathrm{~s}^{-1}$. In the transverse directions, the Nose-Hoover barostat was assigned to maintain $1 \mathrm{~atm}$ of pressure to enable Poisson contractions. A timestep of $0.1 \mathrm{fs}$ and a temperature of $300 \mathrm{~K}$ was specified.

\subsection{Specimen Fabrication and Testing}

Experimental samples of the tetra-functional resin epoxy, TGDDM/DETDA, were prepared to aid in the validation of the MD model. The resin used was Araldite ${ }^{\circledR}$ MY721 (TGDDM), and Aradur ${ }^{\circledR} 5200$ was used for the DETDA hardener. Both the resin and hardener were obtained from Huntsman. The epoxy was prepared by first placing $100 \mathrm{~g}$ of TGDDM and $35 \mathrm{~g}$ of DETDA into separate beakers. The beaker of TGDDM resin was placed in a water bath at $50{ }^{\circ} \mathrm{C}$ to lower the viscosity. While in the water bath, the resin was stirred using a Ross High Shear Mixer (HSM-100 LSKI) with 2 inch dispersion blade. The mixer was set to the lowest speed ( $\sim 500 \mathrm{rpm})$ and the DETDA hardener was then slowly poured into the TGDDM resin. The mixture was mixed at $500 \mathrm{rpm}$ for $10 \mathrm{~min}$ until the two parts were thoroughly mixed. The mixture was then degassed at $70{ }^{\circ} \mathrm{C}$ and $29 \mathrm{inHg}$ with alternating vacuum to avoid overflow until no additional bubbles appeared. The mixture was then poured into a preheated $\left(70^{\circ} \mathrm{C}\right)$ multi-part mold that had been coated with Mann Ease Release 300 and degassed a final time. The curing cycle used was $150{ }^{\circ} \mathrm{C}$ for two hours, 
then heated to $180^{\circ} \mathrm{C}$ at a rate of $2{ }^{\circ} \mathrm{C} / \mathrm{min}$, and held at $180^{\circ} \mathrm{C}$ for two hours. The oven was then turned off, and the cured epoxy was allowed to cool in the oven to room temperature, which resulted in a cooling rate of approximately $1{ }^{\circ} \mathrm{C} / \mathrm{min}$.

The Young's moduli of ten epoxy samples were determined at ambient conditions according to ASTM D638 at a crosshead rate of $1 \mathrm{~mm} / \mathrm{min}$. The test specimens were ASTM Type I sample geometry: $165 \mathrm{~mm}$ long and $3.3 \mathrm{~mm}$ thick. The samples were tested using an InstruMet Sintech screw-driven mechanical testing machine. Stress values were recorded by the testing machine, and an extensometer was used to collect strains. Samples were conditioned at $23{ }^{\circ} \mathrm{C}$ and $50 \%$ relative humidity for 2 days prior to testing.

\subsection{Results}

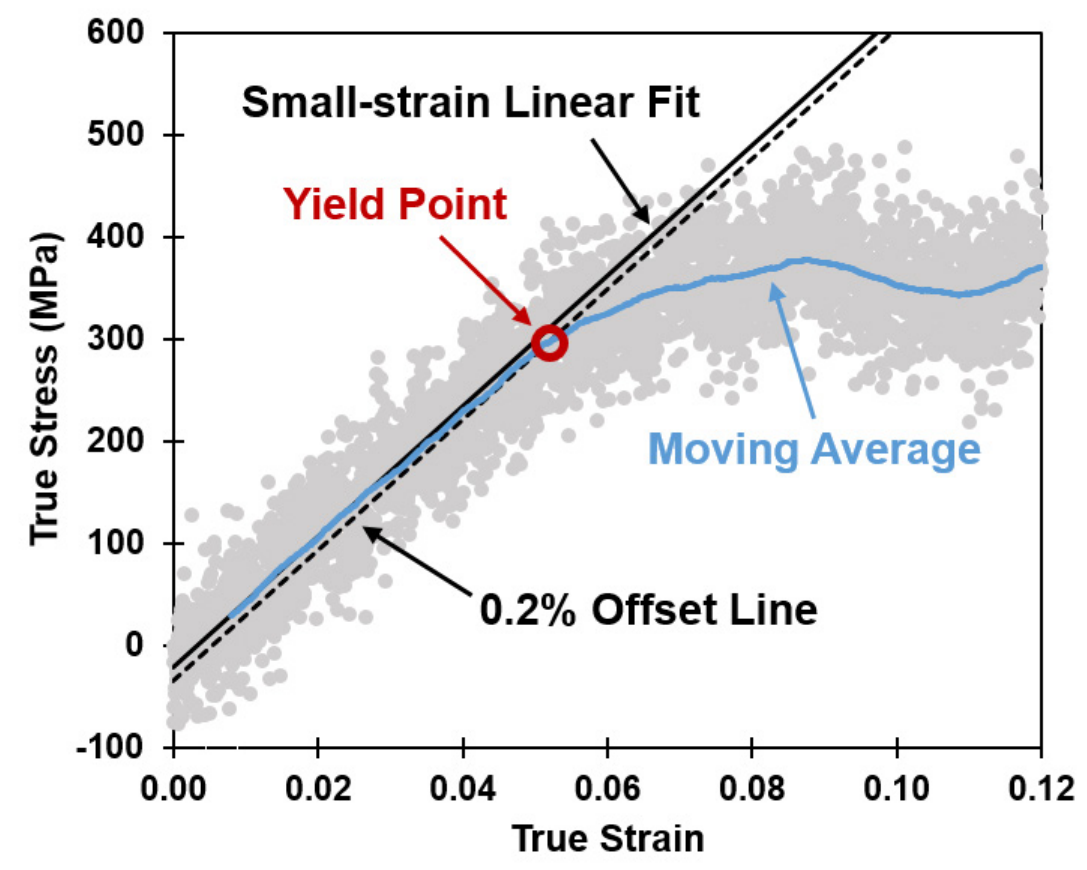

Figure 3-4. Representative stress-strain curve from MD simulation

Stress-strain curves were analyzed for each MD deformation simulation. The true stress and true strain were calculated in the axial direction. To obtain the Young's modulus, the stress-strain data was fit with a linear regression model for strains up to about $3 \%$. A moving average was calculated to smooth the scattered stress-strain data typical of MD models at 
finite temperatures [16]. The smoothing allowed for a more precise determination of the yield point. The yield point was defined as the location where the smoothed stress-strain curve crossed the $0.2 \%$ offset line. A representative stress-strain plot with a linear fit for small strains, the $0.2 \%$ offset line, and the smoothed curve is shown in Figure 3-4.

The predicted Young's moduli from the MD simulations are shown in Table 3-4 for all three epoxy systems. The corresponding uncertainties represent the standard deviation between the five characteristically crosslinked models. Also shown in Table IV are the measured Young's moduli from the above-described experimental tests on the tetrafunctional epoxy, as well as the literature value for the di-functional epoxy from Littell et al. [62]. No literature results for the Young's modulus of the tri-functional resin epoxy could be found since TGAP is commonly cured with 4,4'-diaminodiphenyl sulfone (DDS) rather than DETDA. For both the Di and Tetra epoxies, the values obtained from simulation overpredict the measured value. This can be explained by the stiffening effect of high strain rates that is observed experimentally $[16,62,63]$.

Table 3-4. Comparison of elastic moduli results

\begin{tabular}{|c|c|c|c|}
\hline EPOXY & $\begin{array}{c}\text { YOUNG'S MODULUS } \\
\text { FROM SIMULATION } \\
\text { GPa }\end{array}$ & $\begin{array}{c}\text { YOUNG'S MODULUS } \\
\text { FROM EXPERIMENT } \\
\text { GPa }\end{array}$ & $\begin{array}{c}\text { FLEXURAL MODULUS } \\
\text { FROM EXPERIMENT } \\
\text { GPa }\end{array}$ \\
\hline $\mathrm{Di}$ & $3.51 \pm 0.72$ & $2.89[62]$ & $2.46[64]$ \\
Tri & $4.94 \pm 0.98$ & -- & $2.76 \pm 0.14[31,65]$ \\
Tetra & $5.30 \pm 0.81$ & $3.39 \pm 0.11^{*}$ & $3.04 \pm 0.04[31,65]$ \\
\hline
\end{tabular}

* Young's modulus for the Tetra epoxy was obtained in this work

Figure 3-5 shows the Young's modulus of the di-functional system as a function of strain rate from experimental measurements by Littell et al. [62] and from the present MD modeling. Notice that there is a significant gap between the experimental and computational strain rates. Typical high strain rate testing is performed using the Split Hopkinson Pressure Bar (SHPB) which reaches to the order of $10^{3} \mathrm{~s}^{-1}$. However, due the computational demand of $\mathrm{MD}$, mechanical deformation simulations are limited to even higher strain rates. Currently, it is yet unfeasible for MD simulations to reach the strain rates of the SHPB test. It remains to be seen how experimental and MD mechanical data for epoxies compare at matching strain rates. Nevertheless, Figure 3-5 indicates that the MD prediction generally follows the experimentally set trend. 


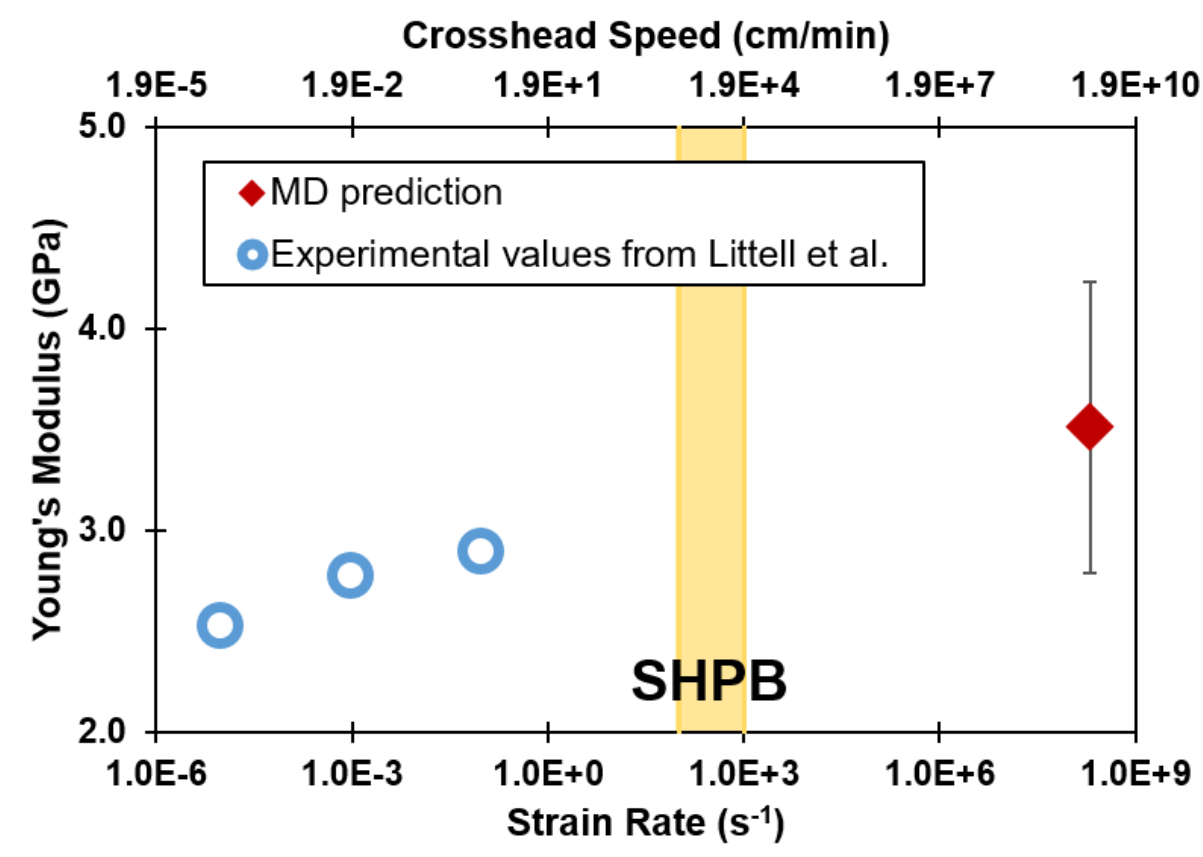

Figure 3-5. Young's modulus versus strain rate for experimental [62] and predicted results for BFDGE/DETDA. Crosshead speeds shown based on experimental setup by Littell et al. (0.125 in. gauge length). Strain rates typifying the Split Hopkinson Pressure Bar test highlighted in yellow.

In spite of the difference in strain rate, the MD simulations correctly predict that the Tetra epoxy is stiffer than the Di epoxy (Table 3-4). Additionally, the molecular modeling of the tri-functional resin epoxy predicts that the Young's modulus will fall between the di- and tetra-functional resin epoxies. The experimentally measured flexural moduli from Becker et al. [31, 66] and Zhou et al. [64] are also shown in Table 3-4 for validation of this trend. For the three epoxy systems studied, the Young's modulus appears to increase with increasing resin functionality.

The average MDIs for the characteristically crosslinked di-, tri-, and tetra-functional resin epoxies were 2.1, 2.8, and 3.1 respectively. The Tri systems have MDIs that are closer to the Tetra models than the Di models due to the higher crosslink densities obtained by the Tri models. Similarly, Table 3-4 indicates that the difference between the average moduli for the Tetra and Tri models $(0.36 \mathrm{GPa})$ is less than the difference between the Tri and Di models $(1.43 \mathrm{GPa})$. While this is not observed for the flexural moduli obtained by Becker et al. [31, 65], this could be due to differences in the extent of crosslinking which was not identified in the study. For the MD models simulated here, the MDI suggests that the highly 
crosslinked Tri networks resemble the Tetra networks which is evidenced by the similar Young's moduli.

In Figure 3-6, the Young's modulus is plotted with respect to the MDI for all models. Each data point indicates the mean Young's modulus of one model, and the standard deviation is shown from three uniaxial tensile simulations. In general, there appears to be a trend of increasing Young's modulus with increasing MDI for all models. However, it is difficult to discern the precise nature of this trend due to the uncertainties observed for these MD models. To elaborate on the observed trend consider the various Tetra models. The lowcrosslinked Tetra models with MDIs of 1.9, 2.2, and 2.3 provided mean Young's modulus values of 3.70, 4.00, and 3.61 GPa respectively. The stiffnesses of the low-crosslinked Tetra models are unlike those observed for the high-crosslinked Tetra models (5.30 $\pm 0.81 \mathrm{GPa})$. However, these low-crosslinked Tetra models have similar MDI values as the highcrosslinked Di models. Unsurprisingly, these low-crosslinked Tetra models possess similar Young's moduli as the high-crosslinked Di models ( $3.51 \pm 0.72 \mathrm{GPa})$.

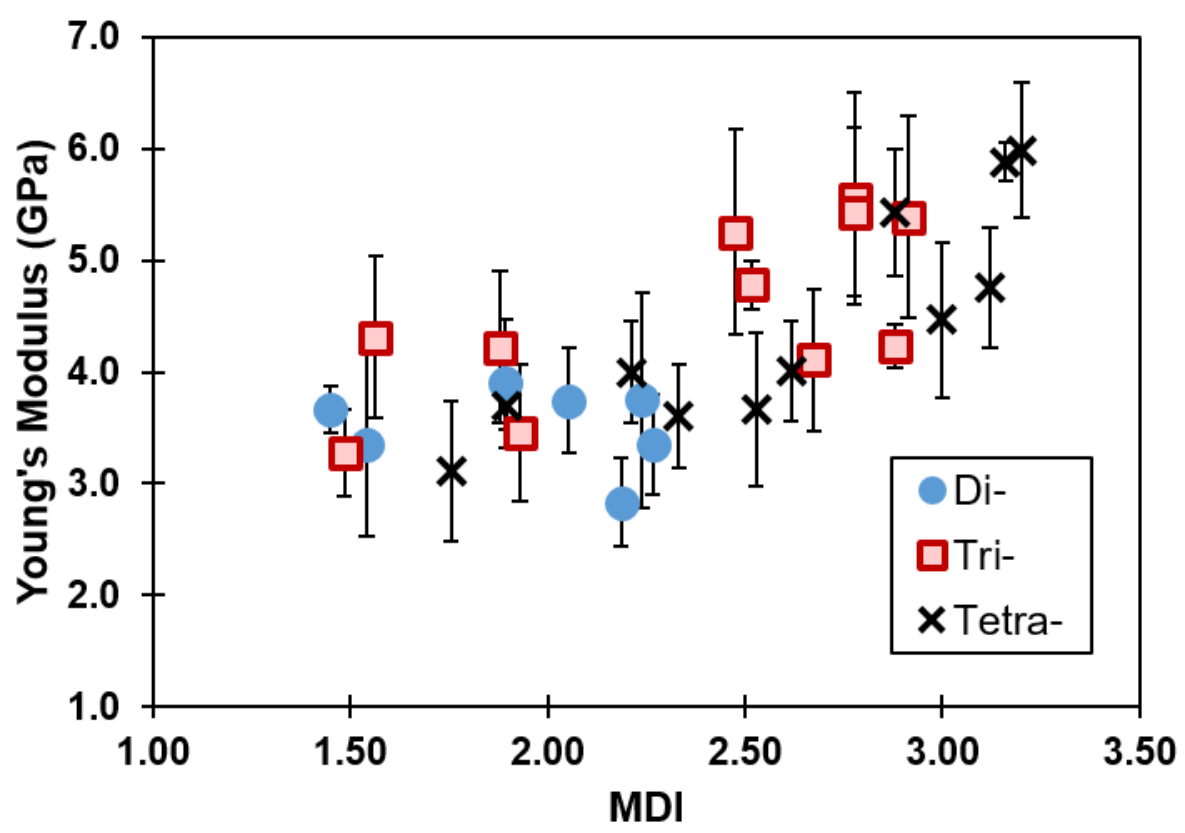

Figure 3-6. Young's modulus versus MDI for all epoxy models.

The yield stress and yield strain results are given in Table 3-5. The yield stress is shown to increase with increasing resin functionality which agrees with previous MD results [48]. 
Comparing the yield stress to experimental results is challenging since the yield stress is known to be especially sensitive to strain rate [62,63]. Figure 3-7 shows the experimental yield stress results for the di-functional resin system [62] using various strain rates and the predicted MD results from this study. It is observed from experimental results that the yield stress increases with increasing strain rate. Thus, it is expected that the values obtained from MD simulation should indeed be higher than experimental measurements.

Table 3-5. Yield point results from simulation

\begin{tabular}{|c|c|c|}
\hline EPOXY & $\begin{array}{c}\text { YIELD STRESS } \\
\text { MPa }\end{array}$ & YIELD STRAIN \\
\hline Di & $135 \pm 36$ & $0.039 \pm 0.011$ \\
Tri & $187 \pm 58$ & $0.039 \pm 0.011$ \\
Tetra & $196 \pm 50$ & $0.037 \pm 0.009$ \\
\hline
\end{tabular}

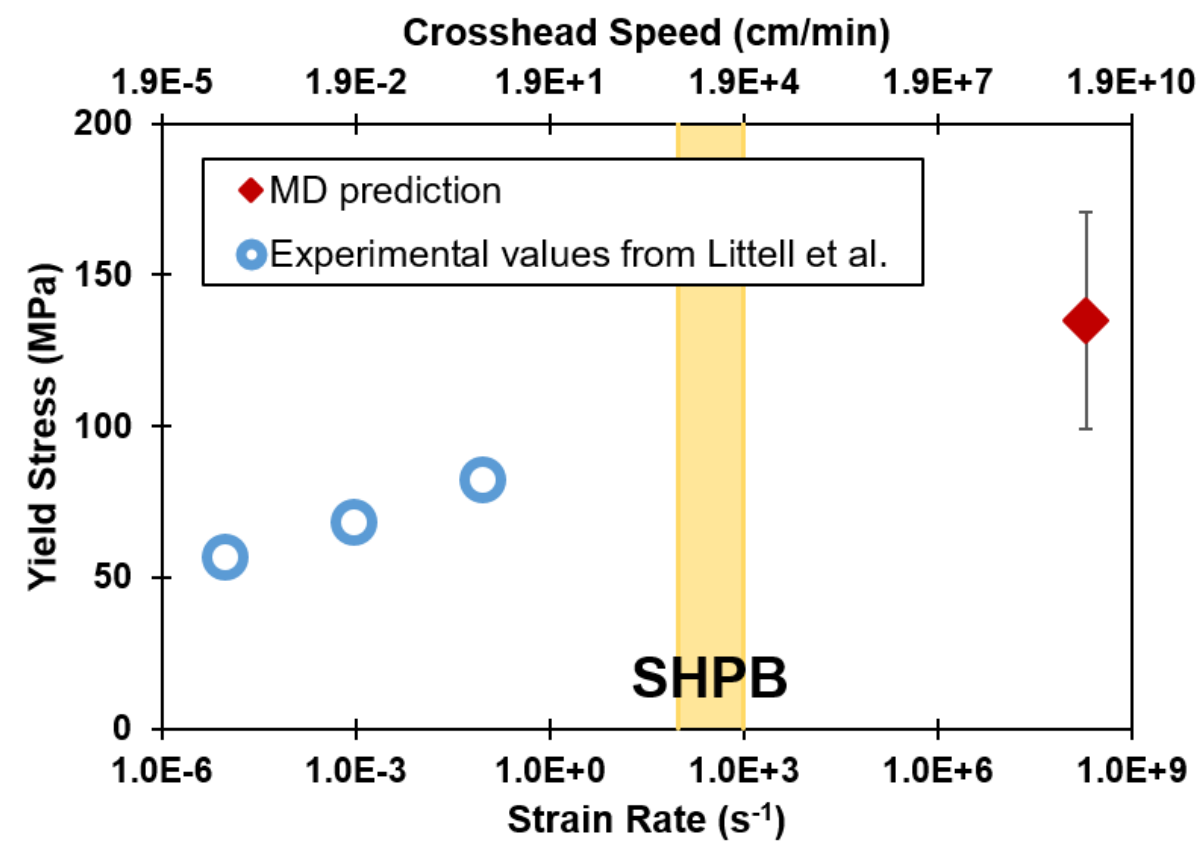

Figure 3-7. Yield stress versus strain rate for experimental [62] and predicted results for BFDGE/DETDA. Crosshead speeds shown based on experimental setup by Littell et al. (0.125 in. gauge length). Strain rates typifying the Split Hopkinson Pressure Bar test highlighted in yellow.

Figure 3-8 shows the yield stress versus MDI for all models. Each data point indicates the average yield stress from deforming an individual epoxy model in three directions. Figure 3-8 suggests a trend of increasing yield stress with increasing MDI. The yield strain 
was consistent for all three epoxy types modeled, and no noticeable effect of the MDI on the yield strain was observed.

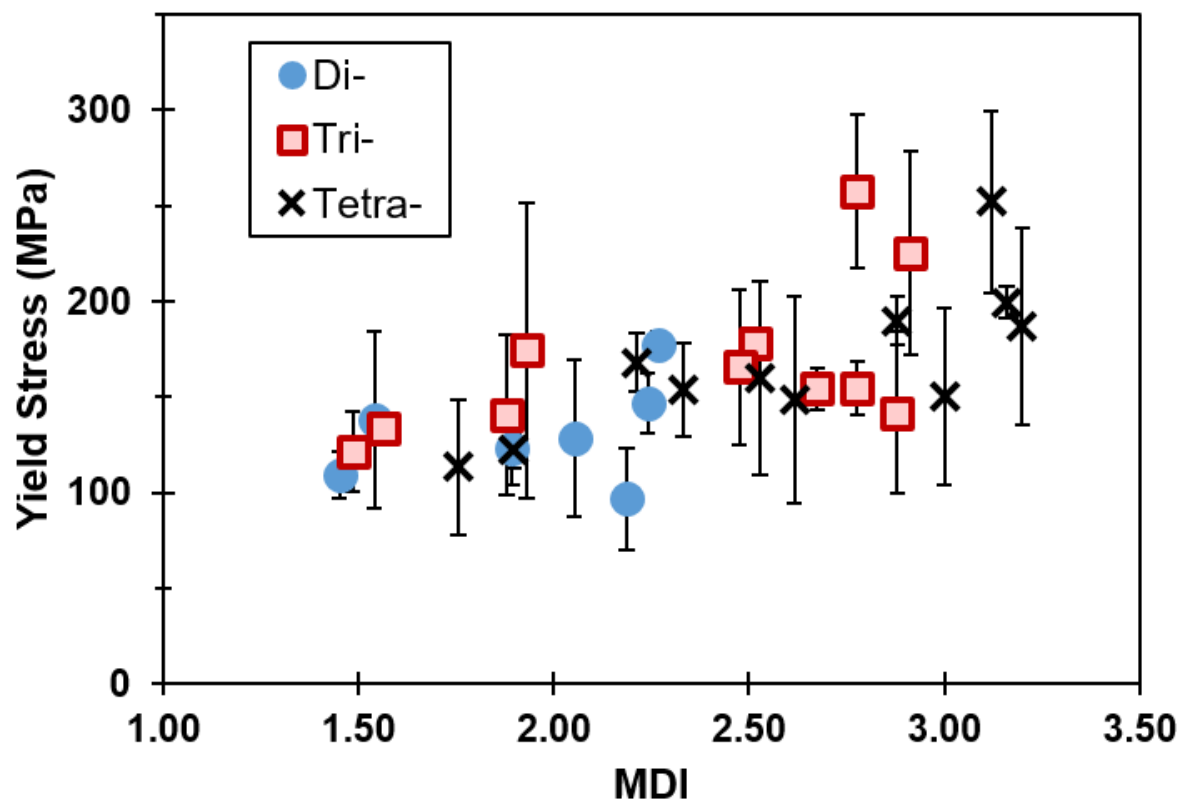

Figure 3-8. Yield stress versus MDI results for all epoxy models.

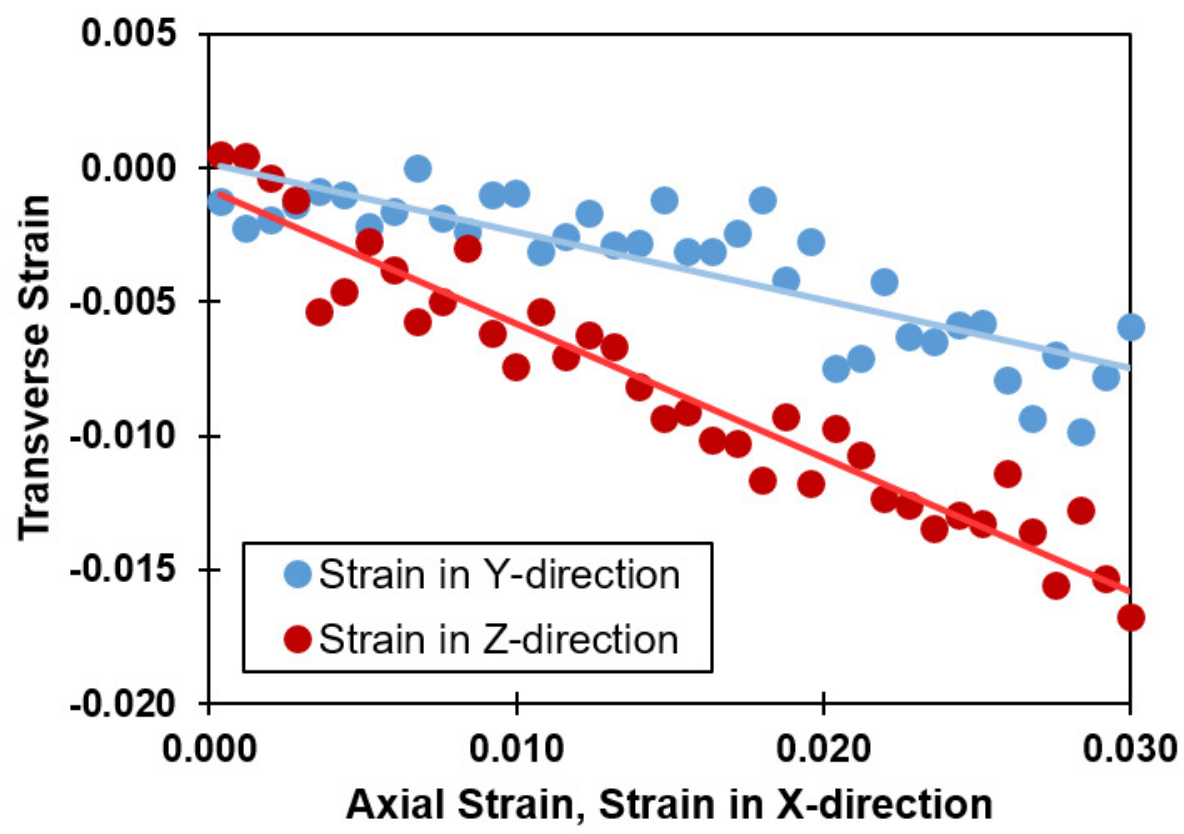

Figure 3-9. Representative transverse strain versus axial strain plot with linear fit. 
The Poisson's ratio was determined from the transverse strain versus axial strain plots as shown in Figure 3-9. A linear fit of for both transverse directions was determined. The negative of the slope of each line was averaged together to get the Poisson's ratio. Table 3-6 gives the Poisson's ratio for each epoxy type. For the di-functional resin epoxy, the Poisson's ratio obtained from experiments were in the range of 0.40-0.43 [62]. The MD simulations give the Poisson's ratio to be $0.37 \pm 0.05$ showing reasonable agreement with experiment considering the Poisson's ratio is independent of strain rate for epoxy [62].

Table 3-6. Poisson's ratio results from simulation

\begin{tabular}{|c|c|}
\hline EPOXY & POISSON'S RATIO \\
\hline Di & $0.37 \pm 0.05$ \\
Tri & $0.37 \pm 0.03$ \\
Tetra & $0.35 \pm 0.04$ \\
\hline
\end{tabular}

\subsection{Conclusions}

The results of this study indicate that the monomer functionality has a significant influence on the Young's modulus and yield stress. Both the Young's modulus and yield stress increased with increasing resin functionality. The experimental literature results reveal the mechanical property rate-dependence. While the difference between the simulation and experimental strain rates is considerable, the MD results generally follow the experimentally set trend. However, it remains challenging to accurately compare the MD mechanical predictions with quasi-static experimental results. Additional mechanical data at strain rates above $10 \mathrm{~s}^{-1}$ is desirable to better link experimental and computational results. The MD models correctly indicate the relative stiffness between the three epoxies studied. This further suggests that ReaxFF with the Liu et al. parameter set is valuable for predicting the stress-strain response of epoxies and understanding structure-property trends. The latter is facilitated by the ability to fully define all details of an MD-modeled polymer network. We demonstrate how characterizing the network junctions allows for informative comparisons between three distinct epoxies by introducing the MDI. Aggregating the mechanical data for all epoxies modeled, the Young's modulus and yield stress is shown to generally increase with increasing MDI. 


\section{Chapter 4 BMI MODELING}

\subsection{Introduction}

The continuous service temperatures of epoxy resins are commonly no greater than $120^{\circ} \mathrm{C}$ leaving much to be desired for high temperature applications. Bismaleimides (BMIs), a key matrix material similar to epoxies, have become an excellent alternative for advanced structural composites due to their superior thermal stability. BMIs typically possess significantly higher glass transition temperatures $\left(T_{\mathrm{g}}\right)$ and heat deflection temperatures than epoxies $[6,67]$. While another well-known high temperature polyimide, PMR-15, presents processing difficulties due to high cure temperatures and its toxic nature [5], BMIs possess an epoxy-like processability [6]. These characteristics, along with excellent toughness and moisture resistance, have earned BMIs substantial appeal in the aerospace industry. Matrimid-5292 is one such commercial BMI polymeric resin for high temperature composites.

The time-temperature cure path is known to affect the overall properties of Matrimid5292. Such an effect has been demonstrated on the $T_{\mathrm{g}}$ by Morgan et al. for both isothermal and stepwise cure cycles [68]. Lincoln et al. analyzed the influence of various monomer ratios and cure cycles on the $T_{\mathrm{g}}$, density, and moisture absorption characteristics of Matrimid-5292 [66]. It was demonstrated that the $T_{\mathrm{g}}$ increases and the density notably decreases with increasing extent of curing for all monomer ratios studied. Using the same monomer ratios and cure cycles, Shin et al. reported on the flexural properties, namely, the flexural modulus, ultimate strength, and flexural strain to failure [69]. It was observed that some degradation of the flexural mechanical properties occurs for extended cure times.

\subsubsection{BMI Cure Reactions}

The cure reactions as well as cure products of Matrimid-5292 have been extensively analyzed using various techniques including: infrared (IR) spectroscopy [68, 70-78], differential scanning calorimetry [68, 70-72, 78], fluorescence and UV-reflectance 
spectroscopy [74], nuclear magnetic resonance (NMR) spectroscopy [76-81], various chromatography techniques [77-79], and mass spectrometry [77-80]. The cure scheme has been proven difficult to characterize, and in spite of the many efforts undertaken [68, 70-81], there is some disagreement on the overall cure mechanism.

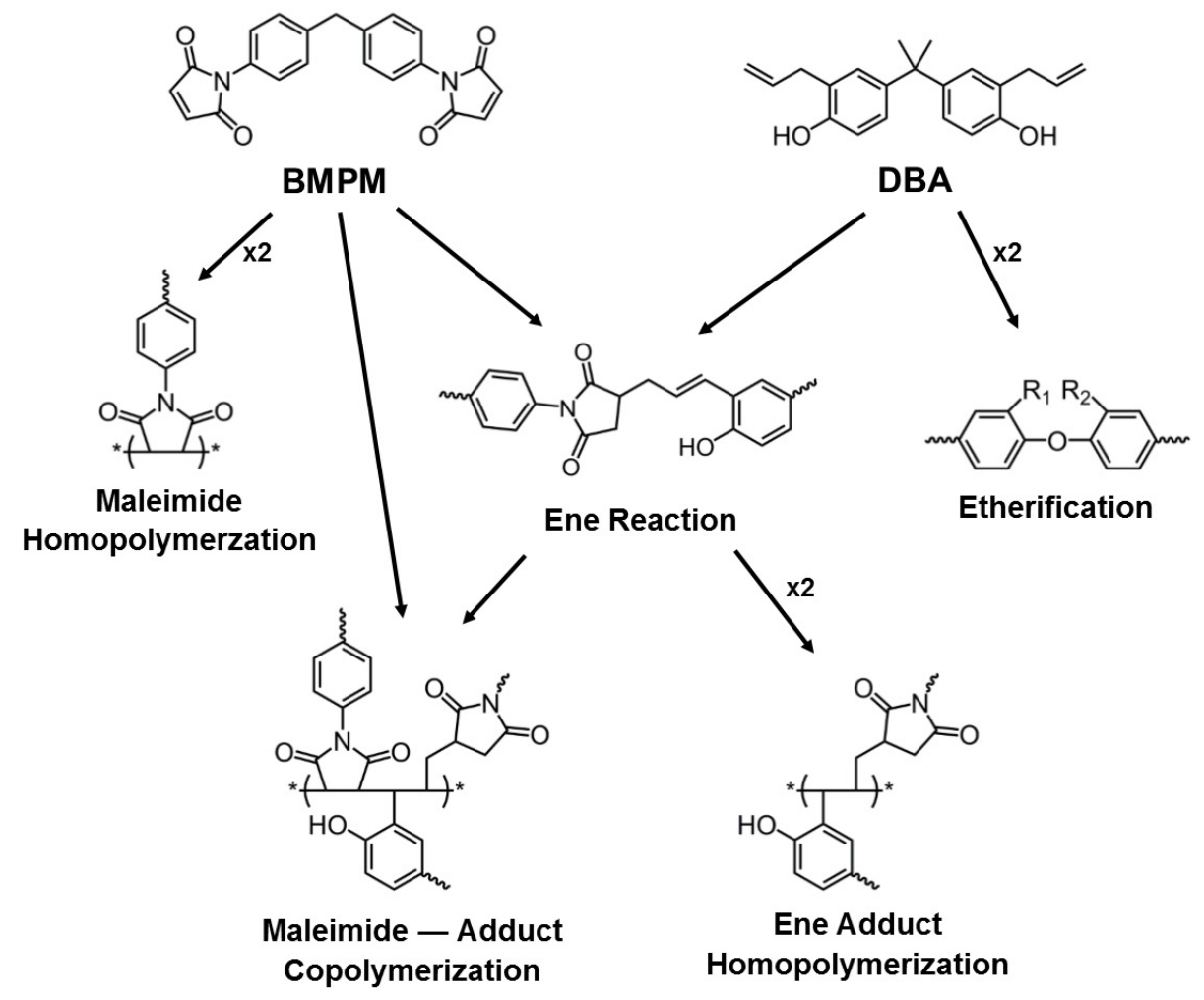

Figure 4-1. Monomer skeletal structures and the proposed cure reactions that were implemented in the present crosslinked Matrimid-5292 model

The skeletal structures of the constitutive monomers, 4,4'-bismaleimidodiphenyl methane (BMPM) and O,O'-diallyl bisphenol A (DBA), and many of the proposed cure reactions are given in Figure 4-1. Among these reactions, almost all of these studies validate the ene adduct and stress the importance of the ene reaction (Figure 4-1) [68, 70-72, 74-81]. The simultaneous consumption of maleimide and allyl groups in an equi-molar system emphasizes the importance of such a reaction. Another proposed mechanism that would lead to equal rates of maleimide and allyl conversion is the alternating copolymerization of maleimide and allyl groups as shown in Figure 4-2. Mijović and Andjelić have claimed that 
this reaction is the primary means for building the BMI network [73]. Other authors have supported alternating maleimide-allyl copolymerization in conjunction with the ene reaction $[71,74,79,81]$. While the ene reaction is largely accepted, there is variance concerning the Diels-Alder reaction (Figure 4-3) between an ene adduct and maleimide group proposed by Zahir et al. [80]. The Diels-Alder reaction has been supported by additional studies in which model monomers are used, namely, N-phenyl-maleimide (PMI) and 2-allylphenol (AP) [77, 82]. However, Shibahara et al. compared the model system PMI-AP with PMI-DBA using ${ }^{13} \mathrm{C}$ NMR spectroscopy and gel permeation chromatography, and while PMI-AP provided for reversible Diels-Alder reactions, this reaction was not observed for PMI-DBA [79]. Rozenberg et al. confirmed the presence of Diels-Alder in PMI-AP and lack thereof in PMIDBA using FTIR spectroscopy and ${ }^{1} \mathrm{H}$ and ${ }^{13} \mathrm{C}$ NMR spectroscopy [76]. The authors suggest that steric hindrances prevent BMPM-DBA from engaging in Diels-Alder reactions.

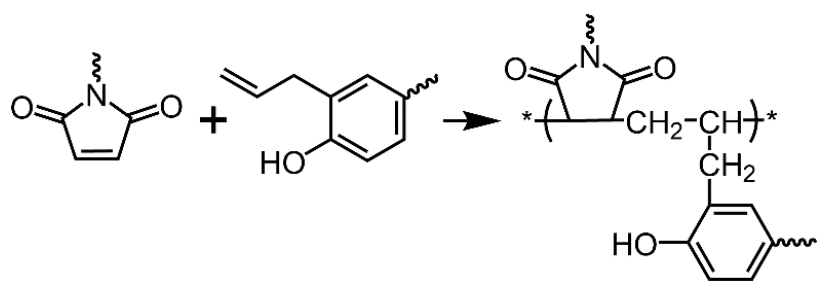

Figure 4-2. Maleimide-allyl copolymerization

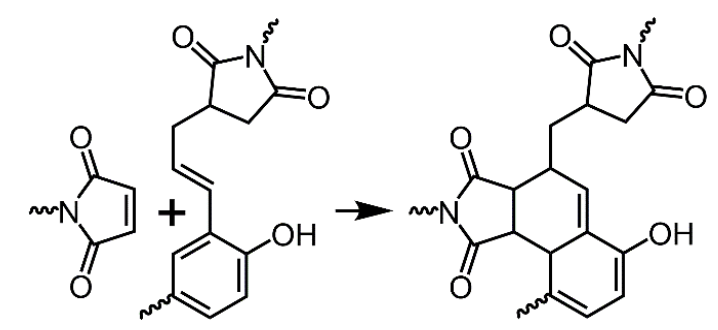

Figure 4-3. Diels-Alder reaction between maleimide and ene adduct.

Additional cure reactions accompanying the ene reaction have been proposed as well. For example, since BMPM is capable of homopolymerizing, the reaction between maleimide groups shown in Figure 4-1 is suggested to be an additional reaction of interest. Etherification of hydroxyl groups (Figure 4-1) which yields water has also been identified in the curing of BMPM-DBA $[68,73]$. Rozenberg et al. have suggested that the ene adduct is 
likely to proceed to polymerize as indicated in Figure 4-1 by the maleimide-adduct copolymerization and ene adduct homopolymerization reactions [76]. After an initial increase of propenyl groups due the formation of ene adducts, a consumption of propenyl is observed which evidences the further polymerization of ene adducts.

Molecular dynamics (MD) provides a molecular perspective and a new opportunity to further investigate the intricacies of BMI crosslinking. Recently, MD models of BMPMDBA have been established by Varshney et al. for analyzing the thermal transport across various carbon fiber/matrix interfaces, but due to the complexities of a crosslinked BMPMDBA matrix as pointed out above in detail, uncured monomer mixtures were modeled in that study $[83,84]$. To the knowledge of the authors, no concerted effort has been invested in modeling cured BMIs, unlike epoxies in which many studies have been undertaken over last decade to model the crosslinked structure [16, 36, 37, 40-46, 85-88]. As noted above, the curing pathways in BMI are markedly more difficult than the curing mechanism of epoxies as a majority of crosslinked MD epoxy models simply incorporate the essential epoxy-amine reaction [16, 36, 37, 40-46, 85-87]. While molecular modeling of crosslinked epoxies has successfully provided an understanding of how the molecular structure affects macroscopic properties, we have not yet seen this level of work carried out for BMIs.

The goal of this chapter is to develop a crosslinking framework to incorporate the complex curing chemistry of BMI, build molecular models of crosslinked Matrimid-5292, and predict their thermo-physical properties. Three different cure strategies are employed to study the consequences of different cure reactions proposed in the literature. The results demonstrate the influence of the molecular structure and degree of curing on various properties.

\subsection{Molecular Modeling}

Models of cured Matrimid-5292 containing about 21,000 atoms were developed. The system consisted of a total of 480 monomers at a 1:1 molar ratio of BMPM:DBA. The AMBER GAFF force field and RESP partial charges were used [89, 90]. We adopted the cure scheme laid out by Rozenberg et al. [76, 78]. Figure 4-1 shows all cure reactions 
incorporated in the crosslinked Matrimid-5292 model. To analyze the influence of the reaction mechanism employed, three different reaction schemes were modeled. The reactions utilized in each model are indicated in Table 4-1. Mechanism A consists of the following reactions: ene reaction, maleimide homopolymerization, and etherification. Mechanism B incorporates the ene reaction, ene adduct homopolymerization, and etherification. Lastly, Mechanism C includes all five reactions shown in Figure 4-1.

Table 4-1. Reactions incorporated for each mechanism

\begin{tabular}{|l|c|c|c|}
\hline \multicolumn{1}{|c|}{ REACTION } & A & B & C \\
\hline Ene Reaction & $\mathrm{x}$ & $\mathrm{x}$ & $\mathrm{x}$ \\
Maleimide Homopolymerization & $\mathrm{x}$ & & $\mathrm{x}$ \\
Etherification & $\mathrm{x}$ & $\mathrm{x}$ & $\mathrm{x}$ \\
Maleimide-Adduct Copolymerization & & & $\mathrm{x}$ \\
Ene Adduct Homopolymerization & & $\mathrm{x}$ & $\mathrm{x}$ \\
\hline
\end{tabular}

The monomers were first arrayed sparsely in a simulation box giving an initial density of $0.3 \mathrm{~g} / \mathrm{cm}^{3}$. A $100 \mathrm{ps}$ simulation was performed to allow the monomers to mix in which the temperature was gradually ramped down from $600 \mathrm{~K}$ to $300 \mathrm{~K}$. Subsequently, the volume of the simulation box was slowly reduced to obtain a density of $1.2 \mathrm{~g} / \mathrm{cm}^{3}$. This densifying simulation was performed at $300 \mathrm{~K}$ for a total of $4 \mathrm{~ns}$ with molecular minimizations conducted every $200 \mathrm{ps}$.

\subsubsection{Crosslinking Algorithm}

The crosslinking algorithm consisted of iterative runs between MD simulations in LAMMPS [50] and topology updates in Python as shown in Figure 4-4. Previous methods have involved the tedious task of manually comparing what new bonds, angles, and dihedrals must be updated and often leads to highly specialized algorithms capable of performing a single reaction. However, our algorithm is more generic due to the on-the-fly comparison between reactants and products and on-the-fly manipulation and update of newly reacted species and its surroundings' force-field parameters (once a new bond formation occurs in LAMMPS), which is made possible by the manner the parameters are stored and applied. Namely, the parameters of the reaction products are pre-stored in a 
LAMMPS data file, an existing file format containing molecule and topology specifications. We will refer to this kind of data file as a product data file. Then in progress, the parameters are applied by creating a one-to-one correspondence from the relevant atoms being simulated to the atoms in the product data file (highlighted rectangle in Figure 4). A product data file is required for each reaction and contains the portion of the product that will undergo a change in atom, bond, angle, dihedral, and improper angle parameters. Some reactions required one product data file, such as the ene reaction; while other reactions required multiple product data files for different scenarios. For example, three product data files were implemented for the maleimide homopolymerization reaction to model chains of succinimides of different lengths: two, three, or more members. Overall 14 product data files were generated. The algorithm was developed to automatically superimpose the parameters from the product data file onto the simulation data file, that is, the actual system of molecules being simulated when the reaction occurs, essentially modifying the molecular parameters of the reactants to the molecular parameters of the products. For this study, we used the AMBER program package to assign parameters and charges, generate all possible product data files, and convert them into LAMMPS format. The framework established herein allowed for multiple reactions to be implemented rapidly with minimal user input. We expect that this framework can be further applied to a wide range of molecular systems.

For each iteration, BMI was equilibrated and new reactions were initiated in LAMMPS. Here, new bonds are created using the 'fix bond/create' LAMMPS command to initiate crosslinking reactions. The bond cutoff distance was set to $6 \AA$. All reactions were assumed to have an equal probability of reacting when the reactive groups were within the bond cutoff distance. Thus, the proximity of reactive groups governed the amount of reactions performed of each type. Using Python, the topology information was updated to complete each reaction. Following the topology update, a 5 ps run was executed using the 'nve/limit' command to moderate the energy from topology changes. This allows newly-formed, overstretched bonds to safely be brought to equilibrium distance. Next, an NVT simulation was performed at $650 \mathrm{~K}$. For the first iteration, 100 ps were used. Subsequent hightemperature runs were increased in increments of 100 ps (i.e., equilibration for the $n^{\text {th }}$ 
iteration was performed for $n$ hundred ps). Thus, as mobility decreases during curing, more simulation time is given to let the model reconfigure.

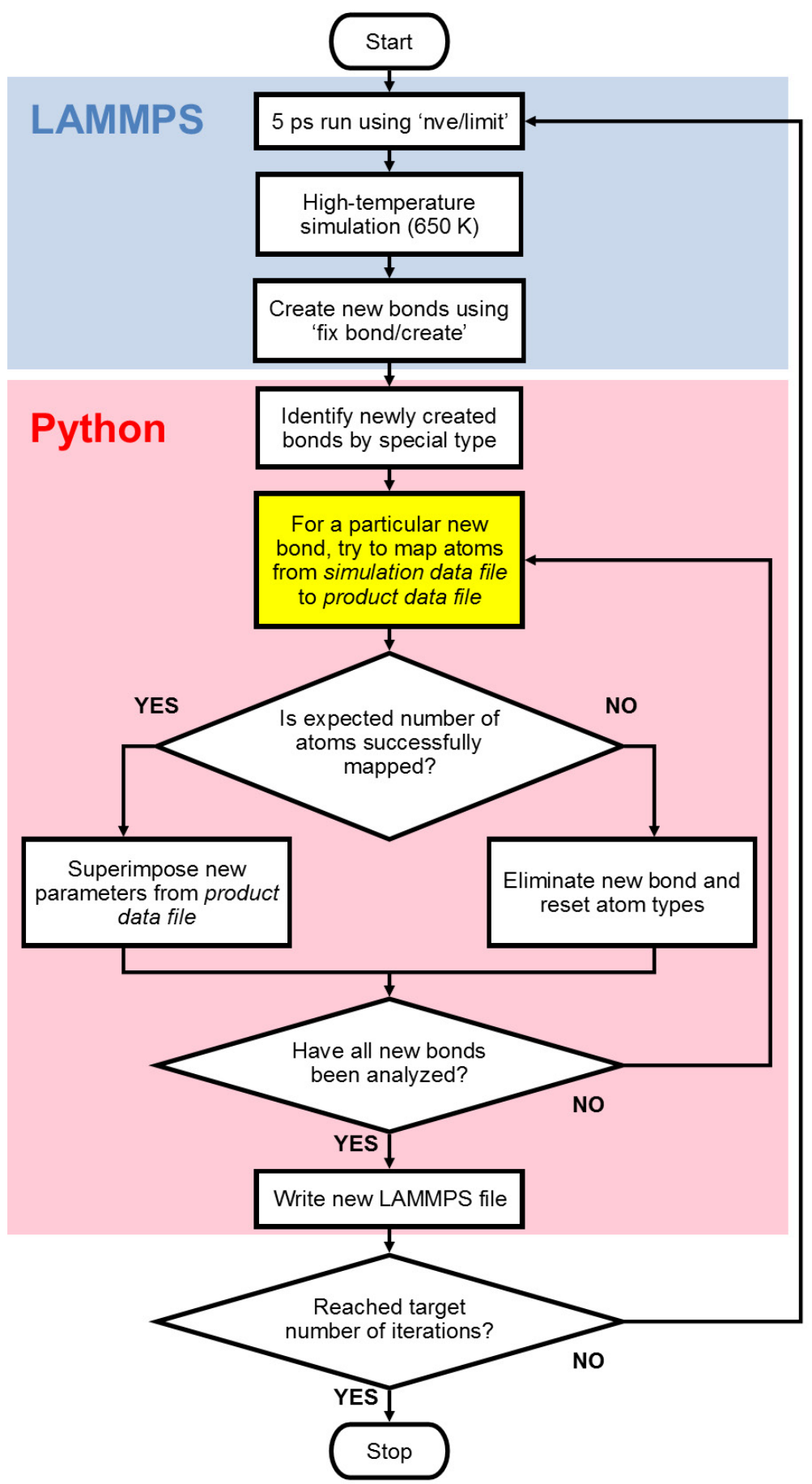

Figure 4-4. Crosslinking flowchart. 


\section{Mechanism A}
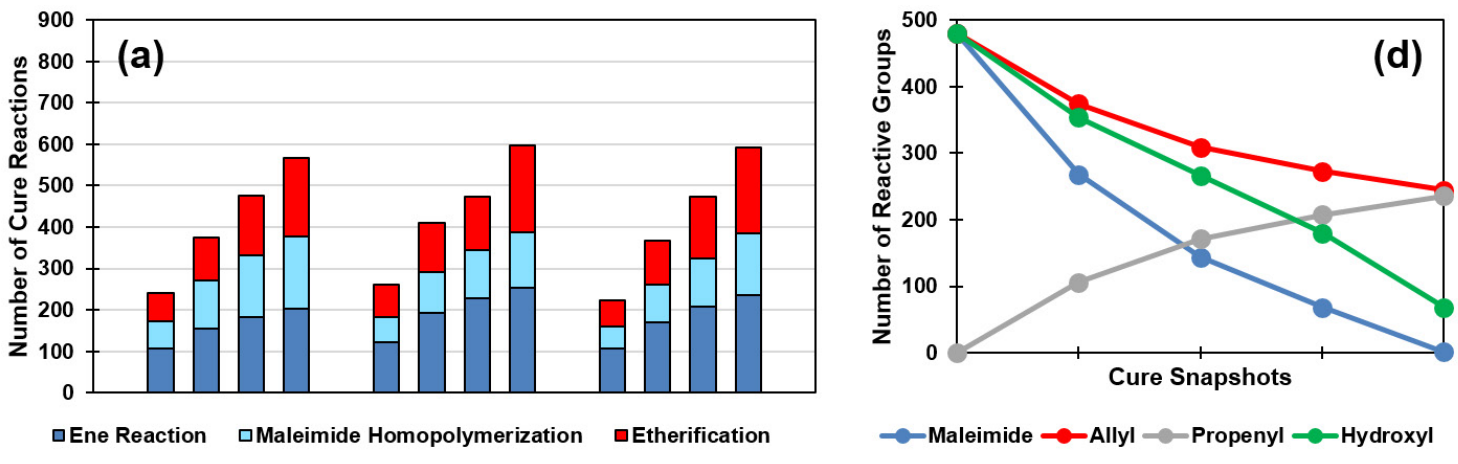

Mechanism B
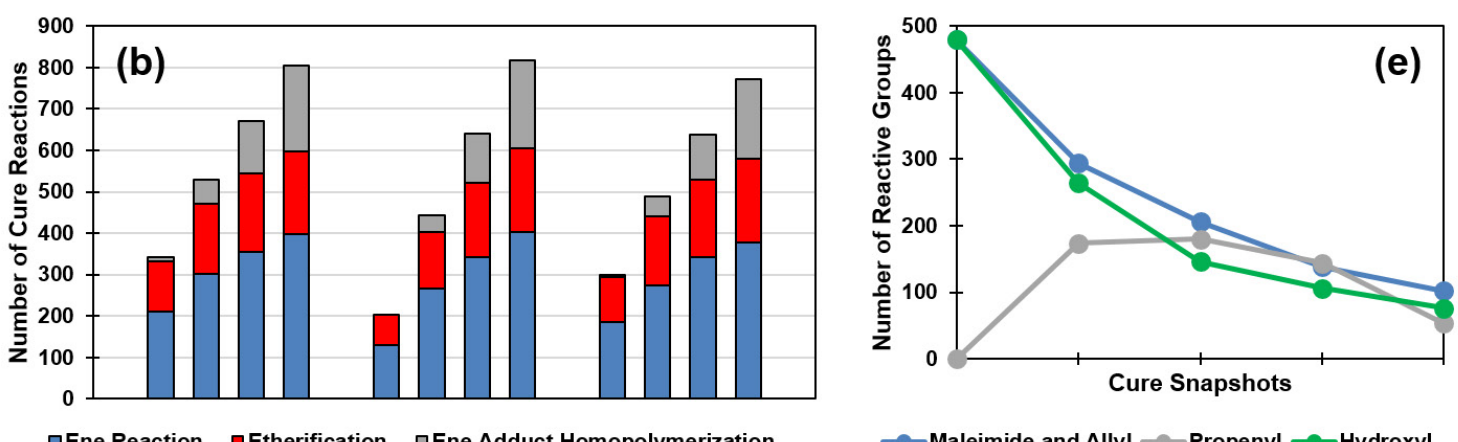

Ene Reaction $\square$ Etherification $\square$ Ene Adduct Homopolymerization

$\multimap-$ Maleimide and Allyl $\multimap-$ Propenyl $\multimap-$ Hydroxyl

Mechanism C
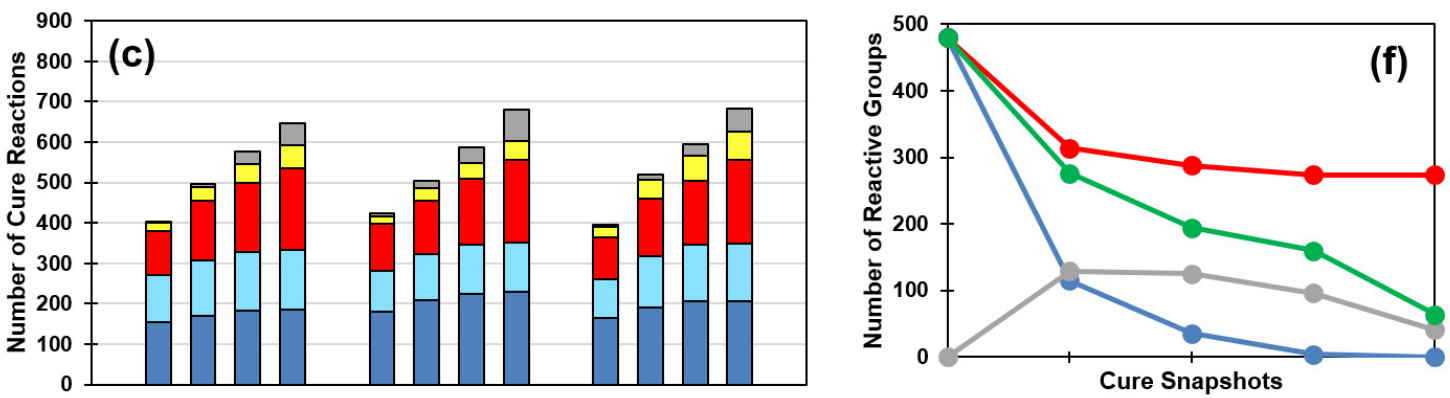

口Ene Adduct Homopolymerization

Maleimide - Adduct Copolymerization 口Maleimide Homopolymerization

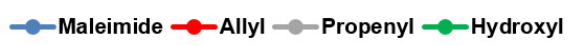

口Etherification

口Ene Reaction

Figure 4-5. (a)-(c) Stacked bar charts indicating the number of cure reactions executed for each cure mechanism in Table 1. Each set of bars shows 4 stages of a particular cure path (3 independent sets). (d)-(f) Number of reactive groups for progressive cure snapshots.

For each cure mechanism, four different crosslinked models were obtained from one monomer sample by selecting models from different stages of curing. All reactions besides 
etherification were performed simultaneously, and an equal number of iterations of etherification were executed separately to avoid complications in parameter changes.

Based on the developed crosslinking algorithm, the number of cure reactions of each type is shown in Figure 4-5 (a)-(c). For Mechanism A, 12 models are shown which are arranged in sets of 4 to represent the cure progression of 3 different and independent monomeric sets. Of this particular reaction mechanism, the least cured and greatest cured models contained 222 and 591 total reactions, respectively. Mechanism B yielded the highest number of reactions (up to 817 reactions); whereas, Mechanism C obtained 682 reactions.

The number of reactions can be compared with the number of reactive groups for progressive cure snapshots as shown in Figure 4-5 (d)-(f). For Mechanism A and C, the maleimide groups were quickly depleted during curing since they were allowed to react with other maleimides in addition to allyl groups. Moreover, a maleimide group could also react with an ene adduct in Mechanism C. Consequently, the allyl groups were deprived of opportunities to react having no alternate reaction pathway apart from reacting with a maleimide. Many allyl groups were left remaining at the end of the crosslinking procedure. For Mechanism B, the maleimide and allyl groups exclusively react together. An equal number of reactive maleimide and allyl remained at all stages of crosslinking. Mechanism B produced more ene adducts than any other mechanism which led to a significant number of ene adduct homopolymerization reactions. Thus, this scheme resulted in the greatest number of reactions. Moreover, Mechanism B reproduces the simultaneous maleimide-allyl consumption experimentally observed in equi-molar systems $[68,76]$.

\subsubsection{Annealing and Deformation Simulations}

All 36 crosslinked BMI models were subjected to annealing simulations. Each model was gradually cooled from 750 to $250 \mathrm{~K}$ over $25 \mathrm{~ns}$, giving a cooling rate of $20 \mathrm{~K} / \mathrm{ns}$. The NPT ensemble was used to observe the density and volume over the entire temperature range. From the annealing simulations, the $T_{g}$, room temperature density, and the coefficient 
of linear thermal expansion (CLTE) were calculated. Using the NPT ensemble, all models were equilibrated at $300 \mathrm{~K}$ and $1 \mathrm{~atm}$. for $5 \mathrm{~ns}$.

The equilibrated models were subjected to uniaxial tension simulations in the $x$-, $y$-, and $z$ - directions. For each tension simulation, deformation occurred gradually over $5 \mathrm{~ns}$ up to $5 \%$ engineering strain, giving a strain rate of $1 \times 10^{7} \mathrm{~s}^{-1}$. In the transverse directions, the Nose-Hoover barostat was set to maintain 1 atm of pressure allowing for Poisson contractions. The timestep selected was 0.5 fs. The axial stress response up to $3 \%$ engineering strain was fit with a linear regression model to obtain the Young's modulus. Moreover, the transverse strain was analyzed for axial strains up to $3 \%$ and fit with a linear regression model to obtain the Poisson's ratio of the crosslinked networks.

\subsubsection{Heat Flow Simulation}

Additional models containing over 31,000 atoms were built for simulating heat flow. These models consisted of 720 monomers at a 1:1 molar ratio of BMPM:DBA. The same model-building steps were carried out as the previous models with the exception that the dimensions of the simulation box were reduced to 38 by 38 by $228 \AA$ during the densification of the monomer mixture prior to crosslinking. The density after reducing the simulation box was $1.2 \mathrm{~g} / \mathrm{cm}^{3}$. The crosslinking procedure and annealing simulations were carried out in the same manner as described previously. After annealing, the final simulation box sizes were roughly $4 \mathrm{~nm}$ in the $x$ - and $y$-directions and $21 \mathrm{~nm}$ in the $z$-direction. The slab-like cuboid as noted above, instead of a cube, was required to carry out heat transport simulations using the Fourier law approach.

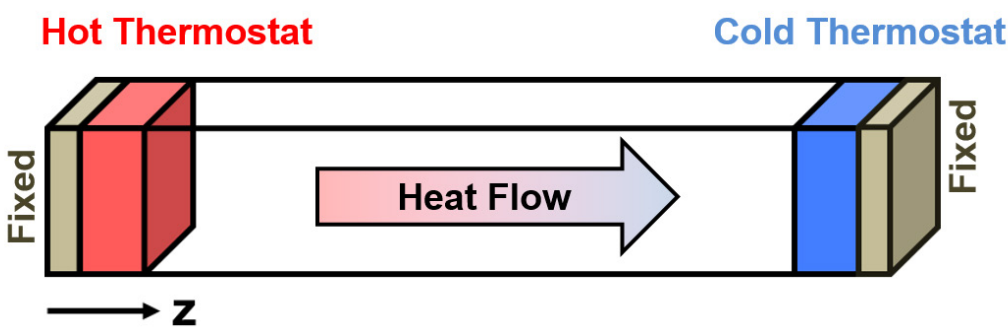

Figure 4-6. Schematic of heat flow simulation. 
The fixed-volume heat flow simulations were then set up by designating a hot and cold region $(20 \AA)$ along the $z$-direction which were maintained at $350 \mathrm{~K}$ and $250 \mathrm{~K}$, respectively, by Nose-Hoover thermostats. No thermostat was applied to the center region. Instead, the NVE ensemble was assigned to allow for a temperature gradient to be established between the hot and cold regions. An additional $20 \AA$ region spanning the periodic boundary in the $z$-direction was specified in which the atoms were fixed to allow the heat flow from hot to cold thermostat only along one direction. A schematic of this setup is given in Figure 4-6. All heat flow simulations were performed for 2.5 ns with a timestep of $0.5 \mathrm{fs}$.

\subsection{Results}

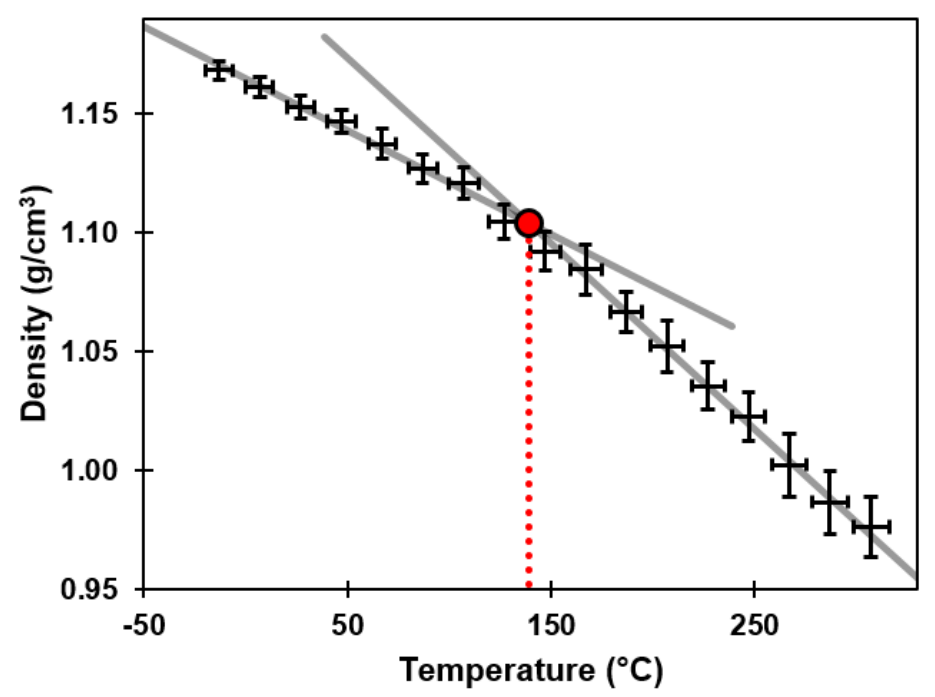

Figure 4-7. Representative density-temperature plot fit with bilinear regression model.

The density-temperature plot obtained from annealing was fitted with a bilinear regression model using the 'segmented' package in R [91]. From an initial estimate, the optimal breakpoint was calculated which was taken to be the $T_{\mathrm{g}}$ as shown in Figure 4-7 for a representative case. The density at $300 \mathrm{~K}$ was estimated from the glassy region line of the bilinear regression model. Additionally, the volume-temperature (V-T) plot was fit with a 
cubic polynomial regression model in order to obtain the temperature-dependent coefficient of thermal expansion $(\alpha)$ at constant pressure as given by,

$$
\alpha=\frac{1}{V}\left(\frac{d V}{d T}\right)_{P}
$$

Figure 4-8 shows a representative volume-temperature graph as well as the slope of the V-T curve at $27^{\circ} \mathrm{C}$ (red) which was calculated from the polynomial model.

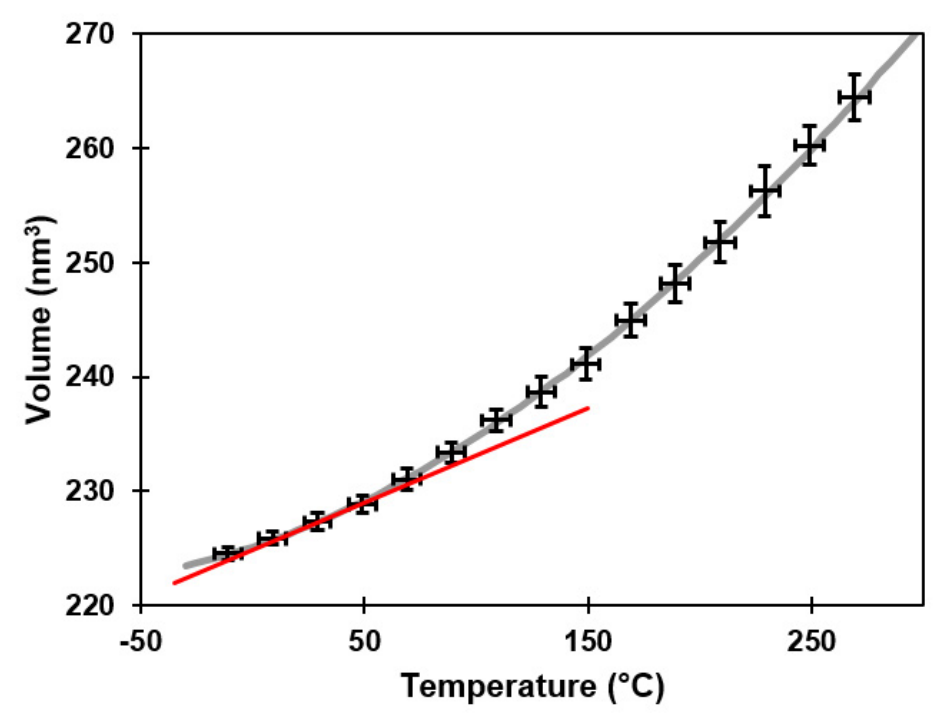

Figure 4-8. Representative volume-temperature plot fit with a polynomial regression model and the slope of the volume-temperature curve at $27^{\circ} \mathrm{C}$ (red).

A common metric used in MD studies to estimate the crosslink density is called degree of curing which can be defined as the total number of cure reactions realized divided by the maximum number of theoretically possible reactions. However, for the current study, determining the maximum number of possible reactions is difficult due to the multiple cure pathways represented in Figure 1. Moreover, it is likely that the maximum number of reactions will differ between the three BMI cure mechanisms modeled. In order to better gauge the degree of curing and compare the three reaction schemes, we select a measure that does not depend on the number of possible reactions. Here, we define a term monomeric 
degree index (MDI) as the average number of 'other' monomeric units covalently bonded to a particular monomeric unit. This average is taken over all monomeric units in the MD system. Practically, the monomeric degree index is calculated as,

$$
\text { MDI }=\frac{2 \times \text { Number of Cure Reactions }}{\text { Number of Monomers }}
$$

In order to exemplify this measure, consider a cyclic homopolymerization system, where each monomer can react with two other monomers. In such a case, for an $n$ monomer system where $n$ reactions take place (creating a cyclic polymer), the MDI value of will be 2, i.e., each monomer is covalently bonded to two other monomers.

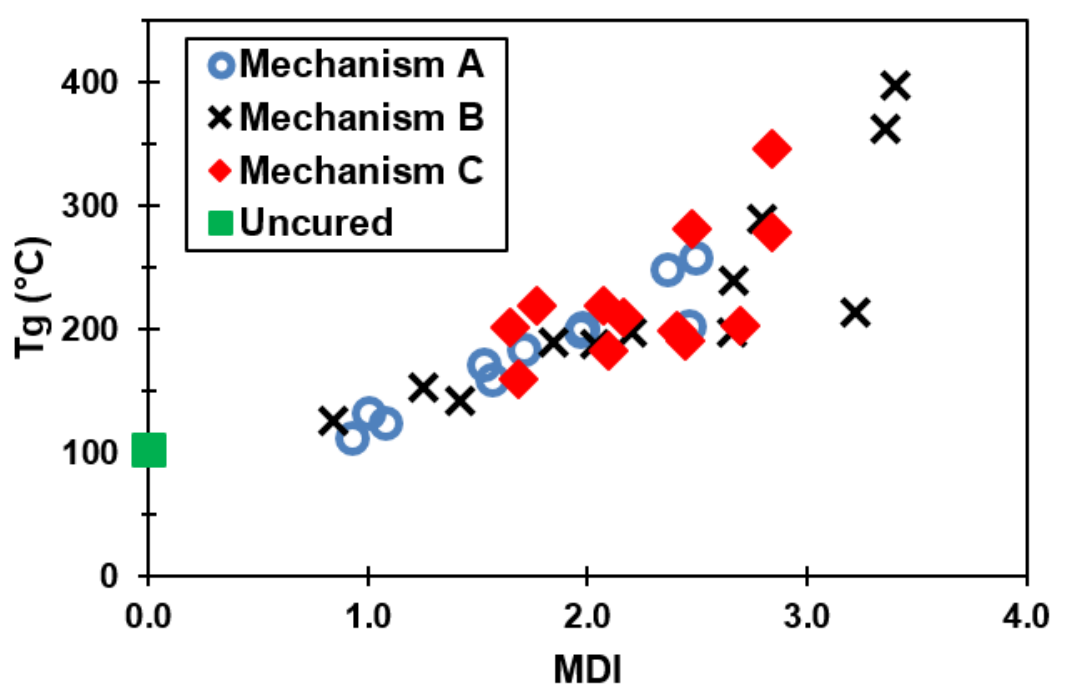

Figure 4-9. $T_{g}$ results from MD annealing simulations.

The $T_{\mathrm{g}}$ results obtained from MD annealing simulations are plotted with respect to the MDI in Figure 4-9. As indicated by the MDI, $T_{\mathrm{g}}$ increases with increasing extent of curing for all cure mechanisms. This trend of increasing $T_{\mathrm{g}}$ with increasing curing has been experimentally measured by Lincoln et al. [66] In the same study, the $T_{\mathrm{g}}$ for an equi-molar system cured at a standard cure cycle was measured to be $313^{\circ} \mathrm{C}$. The Mechanism A models do not reach this value, obtaining a maximum $T_{\mathrm{g}}$ of $202^{\circ} \mathrm{C}$ which is $35 \%$ less than the target 
value. For Mechanism $\mathrm{B}$ and $\mathrm{C}$, the models are able to exceed the target value demonstrating that the experimental measurement falls within the range of values produced by these models. Mechanism B gives the highest $T_{\mathrm{g}}$ values of 397 and $362{ }^{\circ} \mathrm{C}$, and Mechanism C reaches $347^{\circ} \mathrm{C}$.

We should point out that it is extremely difficult to have a one-to-one comparison with respect to the experimental values because it is impossible to mimic the 'standard' cure cycle in simulations. The timescales of performing crosslinking in experiments and simulation differ by almost 8-10 orders of magnitudes, although such a difference may be resolved by using the time-temperature superposition principle with respect to the $T_{\mathrm{g}}$ prediction. Additionally, the experimental reaction rates are dissimilar for different reactions and are functions of temperature, unlike our crosslinking procedure where all reactions are treated as equally likely. Nevertheless, the predicted results provide a degree of validation regarding the methodology as $(a)$ an increase in $T_{\mathrm{g}}$ is observed with greater degree of crosslinking, and $(b)$ the predicted values of $T_{\mathrm{g}}$ are well within the experimentally reported values.

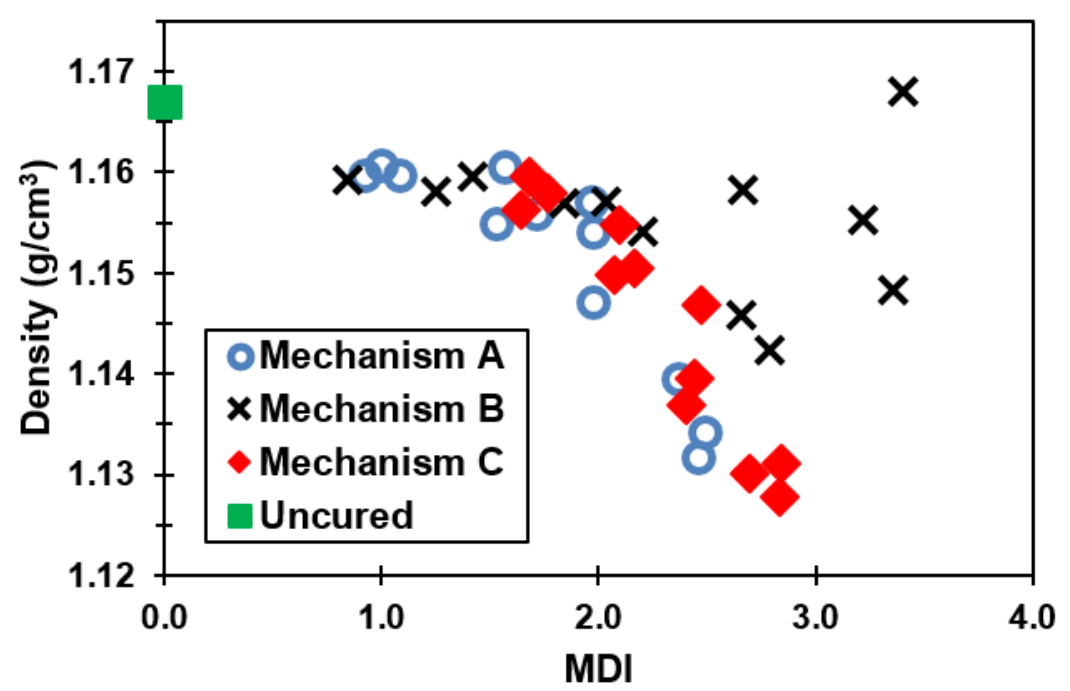

Figure 4-10. Room temperature density results.

The room temperature density results in Figure 4-10 demonstrate an unintuitive trend of decreasing density with increasing extent of curing. Our MD models correctly predict this 
striking, experimentally-observed trend [66]. This is most clearly observed for Mechanism A and C. Lincoln et al. proposed that the increased rigidity of the BMI network with increasing crosslinking is the cause of the observed density trend [66]. Upon formation of the network, a flexible chain would be more likely to adopt a different confirmation than a rigid chain to fill any newly created voids. Thus, it appears that the steric hindrances to achieving tight molecular packing for BMI as the crosslinking degree increases are correctly captured by our MD models. However, Mechanism B shows appreciable scatter in density for the highly-cured models.

Figure 4-11 shows the coefficient of linear thermal expansion (CLTE), which is observed to be decreasing with increasing MDI. Gao et al. experimentally measured the CLTE to be $66 \mathrm{ppm} /{ }^{\circ} \mathrm{C}$ for Matrimid-5292 with a molar ratio of 1:0.86 BMPM:DBA [92]. There are $12 \mathrm{MD}$ models with CLTE values within $5 \mathrm{ppm} /{ }^{\circ} \mathrm{C}$ of the experimentally reported values. All reaction mechanisms yield models that are in good agreement in experiments. Of these models, the MDI ranges from 1.85 to 2.84 . In addition, Mechanism B models with higher monomeric degree indices (greater than 3.0), show significantly lower CLTE values $\left(42-45 \mathrm{ppm} /{ }^{\circ} \mathrm{C}\right)$.

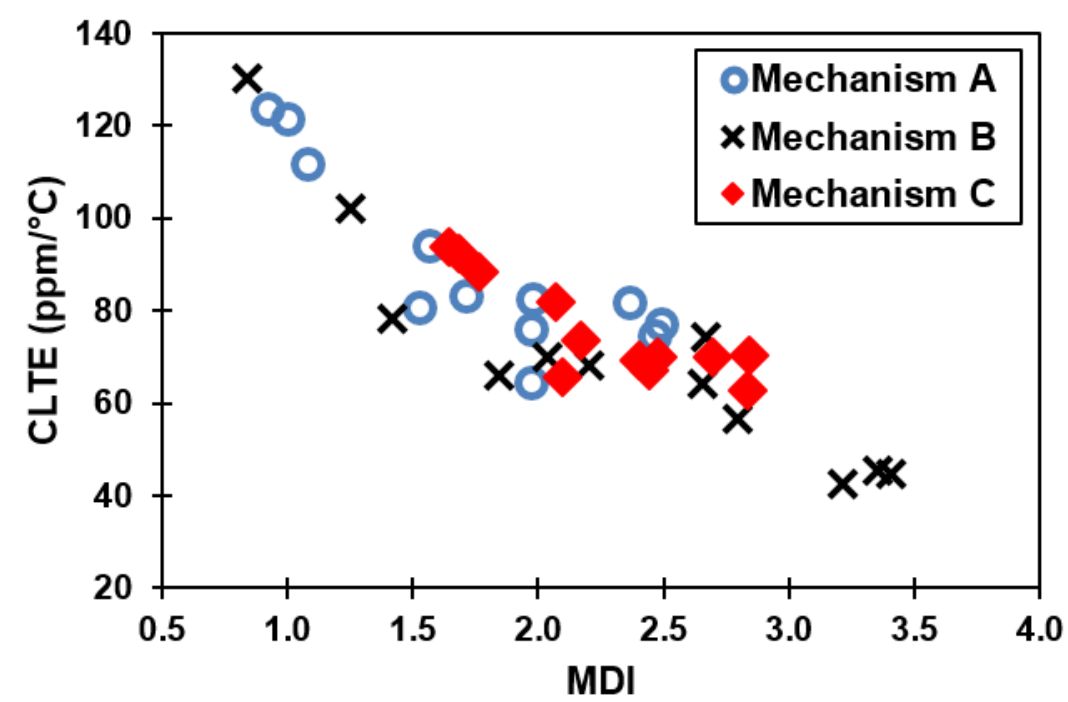

Figure 4-11. MD predictions for the coefficient of linear thermal expansion. 


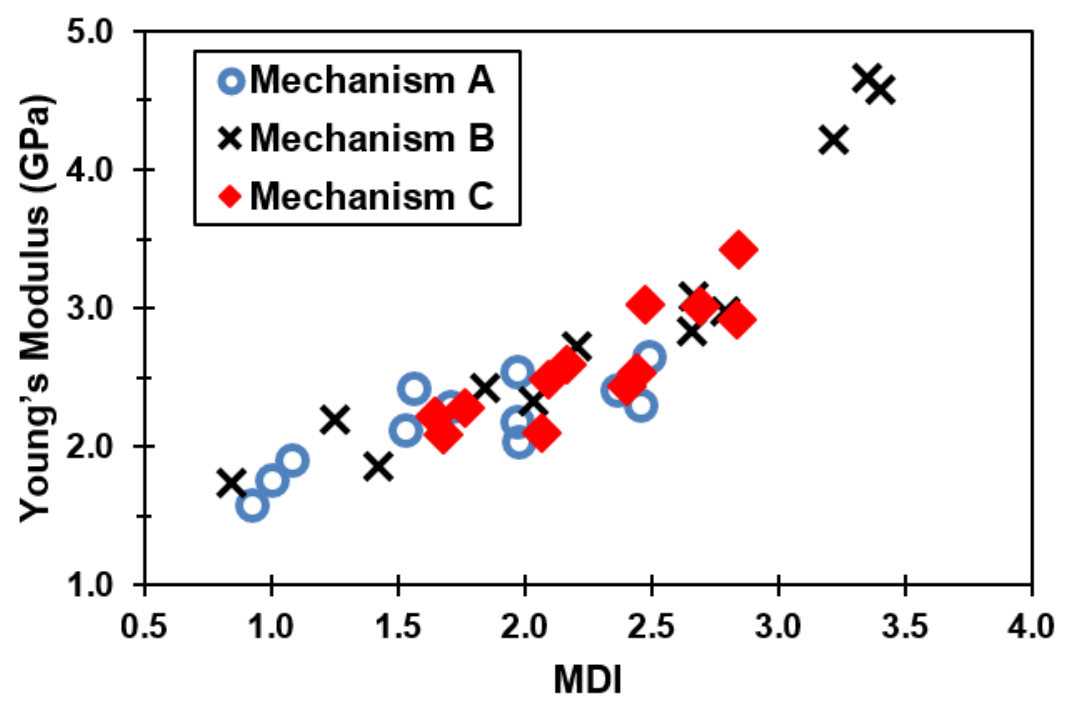

Figure 4-12. Young's modulus results from MD deformation simulations.

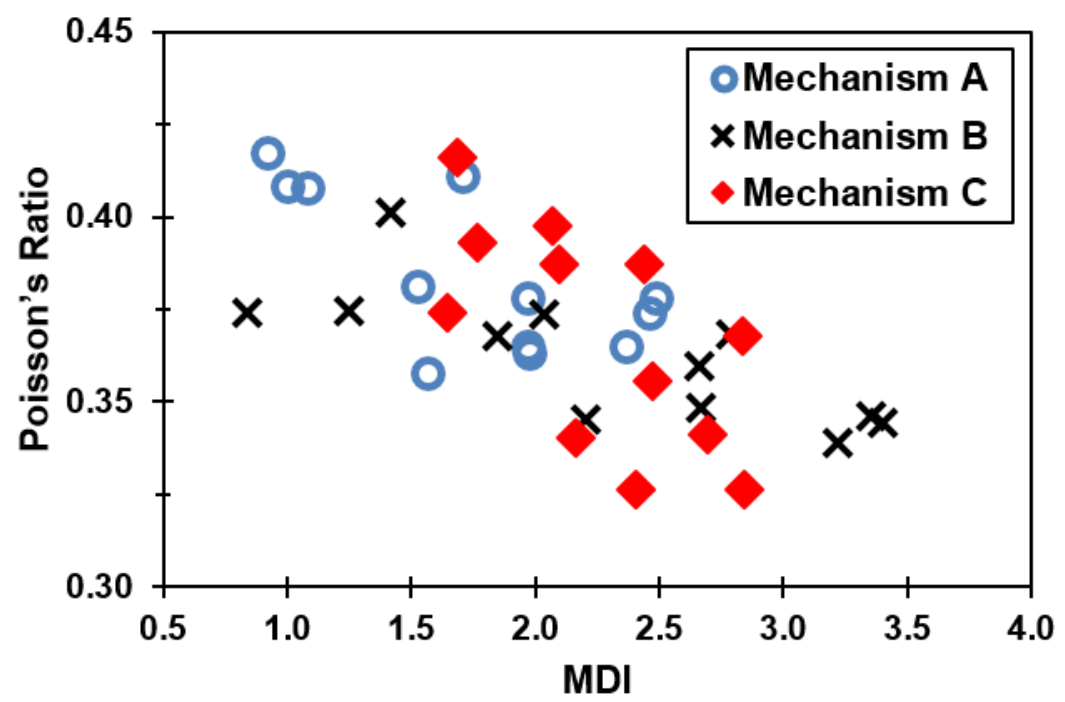

Figure 4-13. Poisson's Ratios obtained for all MD models.

Figure 4-12 shows the Young's modulus values obtained from simulation for all 36 BMI crosslinking cases. The stiffness is seen to increase with increasing MDI. While Mechanism A models showed limited stiffness (up to $2.64 \mathrm{GPa}$ ), Mechanism $\mathrm{C}$ resulted in greater Young's Modulus values, obtaining up to $3.43 \mathrm{GPa}$. However, the Mechanism B models produced the highest moduli: 4.22, 4.57, and 4.66 GPa. Experimentally, King et al. 
measured the tensile modulus of BMI with equi-molar BMPM:DBA formulation to be 4.3 GPa [67]. When comparing simulation models with experimental data, the highly cured Mechanism B models best agree with the experimentally measured Young's Modulus; whereas, the remaining mechanisms yield relatively compliant models. The Poisson's ratios produced from uniaxial tensile deformation simulations are shown in Figure 4-13 and appear to weakly decrease with increasing MDI.

MD predictions of the thermal conductivity were obtained from heat flow simulations using,

$$
\lambda=\frac{\frac{Q}{A \Delta t}}{\frac{d T}{d z}}
$$

where $Q$ is the heat energy, $A$ is the cross-sectional area of the simulation box perpendicular to heat flow direction, $\Delta t$ is the change in simulation time, and $d T / d z$ is the steady-state temperature gradient. Thus, $Q /(A \Delta t)$ gives the heat flux across the center region. For both the hot and cold region, the cumulative energy change due the thermostat was observed. Steady-state was identified when the cumulative energy change became linear with simulation time. The slope of this steady-state line for the hot and cold regions was averaged together to obtain the heat flux $Q /(A \Delta t)$. All models achieved steady-state by $1 \mathrm{~ns}$, and the remaining simulation time was used for obtaining the time-averaged temperature profile. A representative temperature profile is shown in Figure 4-14. The temperature gradient was obtained by fitting the center region with a linear regression model.

The thermal conductivities computed from all MD heat flow simulations are shown in Figure 4-15. The thermal conductivity is seen to increase with increasing MDI. The greatest cured models for Mechanism A, B, and C obtained thermal conductivity values of 0.286 , 0.319 , and $0.330 \mathrm{~W} / \mathrm{m} / \mathrm{K}$, respectively. The latter is nearly double the thermal conductivity of the uncured model $(0.168 \mathrm{~W} / \mathrm{m} / \mathrm{K})$. Gao et al. measured the thermal conductivity to be $0.42 \mathrm{~W} / \mathrm{m} / \mathrm{K}$ for Matrimid-5292 with a 1:0.86 molar ratio of BMPM:DBA [92]. While the thermal conductivity was found to be increasing with MDI, the highest thermal conductivity achieved by the MD models here is about $21 \%$ less than the experimental value. 


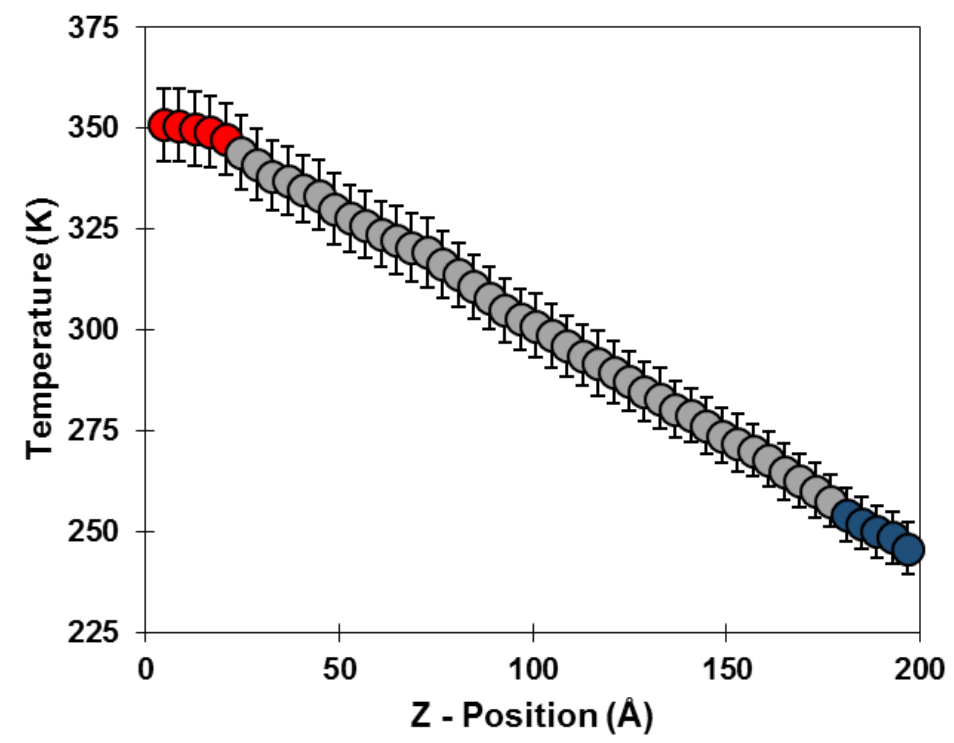

Figure 4-14. Representative temperature profile. The center region is shown in gray between the hot region (red) and cold region (blue).

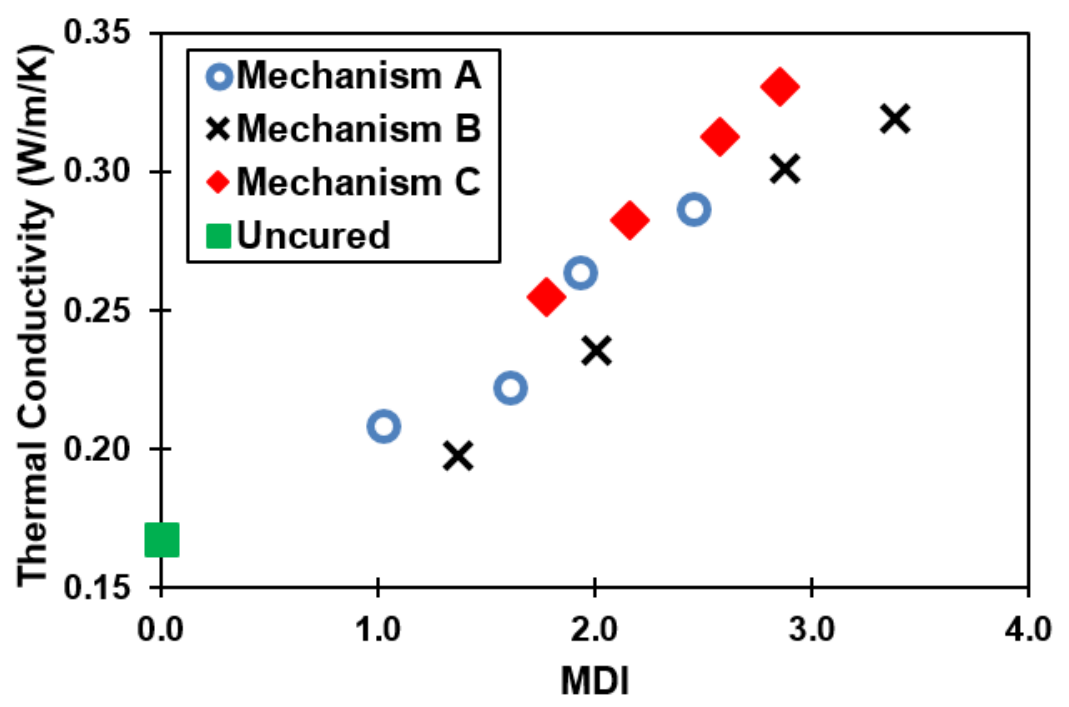

Figure 4-15. Thermal conductivity MD predictions.

\subsection{Conclusions}

A novel LAMMPS-Python crosslinking method has been developed to fully capture the complexities of the BMI network. The developed crosslinked BMI models correctly predict 
experimentally observed trends, namely, increasing $T_{g}$ and decreasing density with increasing extent of curing. By varying the cure reactions and extent of curing, values for the coefficient of thermal expansion, $T_{\mathrm{g}}$, and Young's modulus were obtained that agree well with experimental measurements. Both Mechanism A and C resulted in relatively compliant BMI models, and the consumption of reactive groups was not consistent with experimental results for an equi-molar system. Mechanism B provided more accurate stiffness values and maleimide-allyl consumption.

The developed methods can greatly facilitate the improvement of BMI resins and BMI composite materials. In light of the intricate BMI cure mechanism, predicting the properties based on concentration and curing parameters is challenging. The detailed crosslinked BMI models established in this study can generate new structure-property data to augment experimental studies and assist materials design engineers in tailoring bulk and composite properties as demanded by various applications. Furthermore, these MD models are pivotal in studying nanoparticle reinforcement in BMI resins as they can be combined with models of carbon nanotube, graphene, etc. The methods we outline can also be easily adapted for other thermosetting polymer systems with highly complex curing mechanisms. 


\section{Chapter 5 MULTISCALE CNT/EPOXY MODELING}

\subsection{Introduction}

The growing usage of carbon fiber (CF) composites attests to the demand of stiff-yetlightweight materials. By employing materials with high specific stiffness and specific strength, light-weighting offers such advantages as increased fuel efficiency and lower emissions. Additionally, CF composites can be tailored to handle expected loads. However, these composites presently demonstrate some weaknesses that limit their applicability: low compressive strength, susceptibility to delaminate upon impact, and low thermal conductivity. It is conjectured that the inclusion of nanoparticles in the matrix may address these issues collectively. This multiscale approach may provide a breakthrough to achieving the next generation of CF composites.

The noteworthy mechanical and thermal properties of carbon nanotubes (CNTs) make these nanoparticles attractive candidates for enhancing the mechanical and thermal deficiencies of traditional CF composites. For the success of CNT-enhanced composites, the well-known difficulties of obtaining good CNT dispersion and good CNT/matrix interaction have been addressed extensively. Among the numerous studies conducted, some have sought to understand what matrix characteristics promote good CNT/matrix interaction. Korayem et al. compared two different commercial epoxies used in reinforcing civil structures and found that the more ductile epoxy saw the greater improvement in Young's Modulus for two forms of CNTs, namely CNT powder and CNT masterbatch [8]. To corroborate the nanocomposite stiffness results, the strength of the CNT/matrix interaction was qualitatively assessed using SEM to examine CNTs at the fracture surface, whether the CNTs tended to be extracted from the matrix or fractured. Similarly, $\mathrm{Ci}$ and Bai investigated the effect of the epoxy matrix stiffness by controlling the cure time and the ratio of hardener to resin [9]. It was shown that soft, ductile epoxies demonstrate the greatest improvement in Young's Modulus for $0.5 \mathrm{wt} \%$ of CNT; whereas, no improvement in modulus was observed for the stiffest epoxy used. Additional studies have confirmed that stiff epoxies are not well 
enhanced by CNTs [10-12]. However, this principle is not necessarily true when the CNT is functionalized in order to covalently bond to the matrix [13].

Molecular modeling provides additional insights by simulating the molecular interactions between a polymer and CNT. This computational approach has also been utilized to study what molecular features allow for good adhesion to CNTs. For example, it has been concluded that the presence of benzene [93] and sulfur [30] in the polymer promotes good interaction with CNT. Typically, studies that compare different polymers interacting with CNT are performed with an oligomer and CNT in a vacuum [28-30, 94, 95]. Yang et al. used Molecular Dynamics (MD) interaction energy calculations for CNTs with four different polymers containing benzene rings [28]. Attention was given to the orientation of the benzene rings to the CNT, and it was shown that the polymers that could align its benzene rings parallel to the CNT demonstrated significantly better interaction. It was concluded by the authors that this was due to better $\pi-\pi$ stacking. Additionally, the nonbonded adhesion of different epoxy monomers and curing agents to CNTs have been compared using MD [93, 96, 97]. Wu et al. built uncured models of EPON 862/DETDA and EPON 862/DETA, both with CNT, and determined that the DETDA curing agent demonstrates better interaction with CNT than DETA [93]. While modeling the individual epoxy resin and hardener monomers with CNT can shed light on the initial pre-crosslinked state, the network structure produced upon curing may determine the final adhesion properties. This has been demonstrated by $\mathrm{Li}$ et al. in studying the interaction between an epoxy and silica substrate in which the interaction was shown to increase with increasing crosslink density [98].

The objective of this study is to build MD models of three different crosslinked epoxy systems with CNT and analyze the interfacial and mechanical properties. To predict the effective properties of a realistic CNT/epoxy nanocomposite, a micromechanics approach will be utilized. The modeling results will be compared with experimental moduli obtained in the literature. Similarly, the properties of hybrid CF/CNT/epoxy composites will also be predicted for each epoxy type. At each step of the multiscale method, the dissimilarities of the three epoxy matrices will be discussed. 


\subsection{Modeling}

\section{CODE}

\section{RESIN}

HARDENER

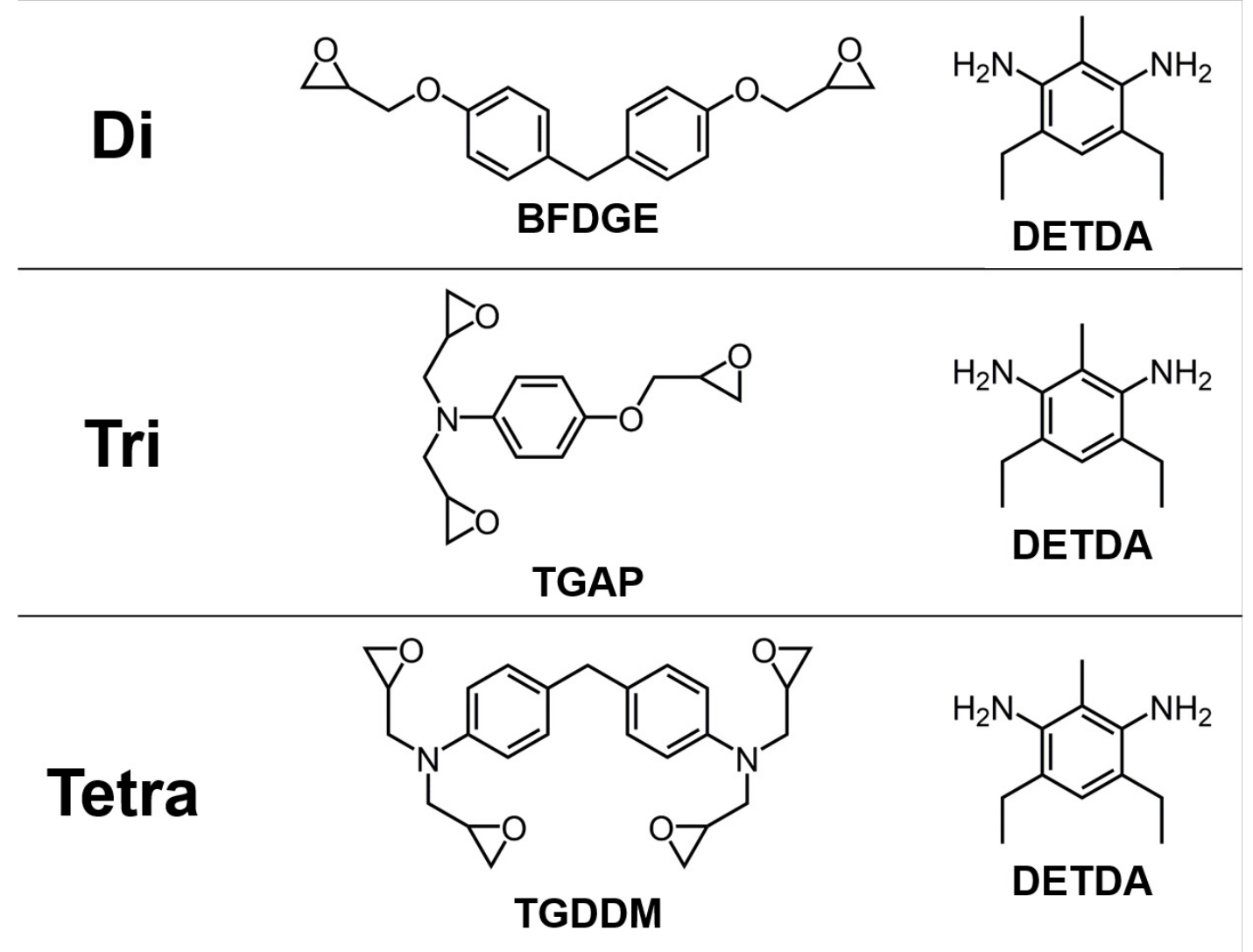

Figure 5-1. Three epoxy systems modeled with CNT and the skeletal structures of each monomer

Three unique epoxy systems were modeled with CNT. Figure 5-1 provides the monomers of each epoxy and the skeletal structures of each monomer. For all systems, the same hardener, diethyltoluenediamine (DETDA), was used. However, various resin monomers were chosen: bisphenol F diglycidyl ether (BFDGE, EPON 862), tri-glycidyl para-amino phenol (TGAP, Araldite MY 0510), and tetra-glycidyl-4,4'diaminodiphenylmethane (TGDDM, Araldite MY 721). Note that the number of reactive epoxide groups is unique for each resin molecule indicating that the functionality of each resin is distinct. The functionalities of BFDGE, TGAP, and TGDDM are 2, 3, and 4 respectively. Thus, we will use the names Di-, Tri-, and Tetra- to refer to BFDGE/DETDA, TGAP/DETDA, and TGDDM/DETDA as indicated in Figure 5-1. 
The ratio of resin monomers to hardener monomers was chosen so that exactly two epoxide groups were present for each amine group. Since each amine group can react with two epoxide groups this provides for a stoichiometric ratio of resin to hardener. For the Di-, Tri-, and Tetra- systems, the ratio of resin to hardener molecules was $2: 1,4: 3$, and $1: 1$ respectively (Table 5-1). The number of resin molecules, number of hardener molecules, and total number of epoxy atoms are also listed in Table 5-1 for each epoxy type.

Table 5-1. Details of epoxy matrix for each type

\begin{tabular}{|c|c|c|c|c|}
\hline EPOXY & $\begin{array}{c}\text { NO. OF RESIN } \\
\text { MOLECULES }\end{array}$ & $\begin{array}{c}\text { NO. OF HARDENER } \\
\text { MOLECULES }\end{array}$ & $\begin{array}{c}\text { RESIN-TO- } \\
\text { HARDENER RATIO }\end{array}$ & $\begin{array}{c}\text { TOTAL NO. OF } \\
\text { EPOXY ATOMS }\end{array}$ \\
\hline $\mathrm{Di}$ & 90 & 45 & $2: 1$ & 5265 \\
$\mathrm{Tri}$ & 84 & 63 & $4: 3$ & 5229 \\
Tetra & 57 & 57 & $1: 1$ & 5244 \\
\hline
\end{tabular}

\subsubsection{Model Construction}

A periodic simulation box was created containing the epoxy monomer models. The OPLS all-atom force field [54] was implemented in LAMMPS [50] for building the monomer models and assembling and cross-linking the nanocomposite system. The initial mass density was $0.09-0.10 \mathrm{~g} / \mathrm{cm}^{3}$. A $100 \mathrm{ps}$ fixed-volume simulation was performed in which the monomers were allowed to mix. Simultaneously, the temperature was ramped down from 600 to $300 \mathrm{~K}$. The 5 independent samples of each epoxy type were distinguished by setting different random initial velocities. Thus, for each sample, the monomers mixed in a unique manner. Thereafter the simulation box was gradually reduced in size to a density of about $1.05 \mathrm{~g} / \mathrm{cm}^{3}$ over $4 \mathrm{~ns}$ with molecular minimizations performed every $200 \mathrm{ps}$. The new box dimensions were determined such that the length in z-direction was $41.6 \AA$ to accommodate the CNT. During densification, the temperature and timestep were set to 300 $\mathrm{K}$ and 1 fs respectively.

A cylindrical void was created by applying the "fix indent" command in LAMMPS during a 100 ps simulation. The radius of the cylindrical region in which atoms would be repelled was increased from 0 to $7.4 \AA$ over the course of the simulation. The volume remained fixed and the temperature was set to $300 \mathrm{~K}$. 
For all models, an unfunctionalized, zigzag $(10,0) \mathrm{CNT}$ was inserted into the void. The resulting nanocomposite models all contained about $12 \mathrm{wt} \% \mathrm{CNT}$ as shown in Table 5-2. The CNT and epoxy monomers were equilibrated for $6 \mathrm{~ns}$ at $300 \mathrm{~K}$ using the NVT ensemble.

The epoxy monomers were crosslinked for $1 \mathrm{~ns}$ at $300 \mathrm{~K}$ while the CNT atoms remained fixed. No covalent bonds were formed between the CNT and epoxy. Further details on the crosslinking procedure is described in a previous work [99]. For each epoxy type, the average crosslink density of 5 samples is given in Table 5-2. Both the Di- and Tetrasystems averaged $74 \%$ crosslink density. However, the Tri- epoxy is able to obtain a higher average crosslink density of 79\%. Previous pure epoxy models also showed higher crosslink densities for the Tri- system [99].

Table 5-2. Nanocomposite models

\begin{tabular}{|c|c|c|c|}
\hline EPOXY & CNT WT\% & $\begin{array}{c}\text { CROSSLINK } \\
\text { DENSITY }\end{array}$ & $\begin{array}{c}\text { DENSITY } \\
\mathbf{g} / \mathbf{c m}^{\mathbf{3}}\end{array}$ \\
\hline Di- & 11.7 & $0.74 \pm 0.04$ & $1.257 \pm 0.006$ \\
Tri- & 12.2 & $0.79 \pm 0.02$ & $1.261 \pm 0.006$ \\
Tetra- & 12.3 & $0.74 \pm 0.02$ & $1.232 \pm 0.008$ \\
\hline
\end{tabular}

Once the crosslinked structure of epoxy was established, the nanocomposite system was switched from OPLS to the ReaxFF reactive force field [15]. The Liu et al. parameter set with low gradient corrections was selected since it demonstrates improved van der Waals interactions for solids [49] and has been confirmed to provide reliable results for the elastic moduli of epoxies $[16,99]$. The hydroxyl groups that were created upon crosslinking were not assigned angle or dihedral parameters in OPLS [99]. As a result, the conformation of some hydroxyl groups were unstable in ReaxFF. This was rectified by allowing the hydroxyl group hydrogens to reposition with limited kinetic energy while all other atoms remained fixed. This was accomplished during a $100 \mathrm{ps}$ simulation using the "temp/rescale" and "fix viscous" LAMMPS commands. The timestep was set to 0.1 fs for all simulations using ReaxFF. Afterwards, all atoms were gradually brought into motion by ramping the temperature from 1 to $300 \mathrm{~K}$ over $100 \mathrm{ps}$.

The ReaxFF CNT/epoxy models were equilibrated over $2.1 \mathrm{~ns}$ at $300 \mathrm{~K}$. Using the NPT ensemble, the Nose-Hoover barostat was set to maintain 1 atm of pressure on all sides of the 
simulation box. Table 5-2 gives the average mass density after equilibration for each epoxy type. The final simulation box dimensions were roughly $35 \times 35 \times 42 \AA$ where the z-direction is the axial direction of the CNT. A completed CNT/epoxy MD model is shown in Figure $5-2$.

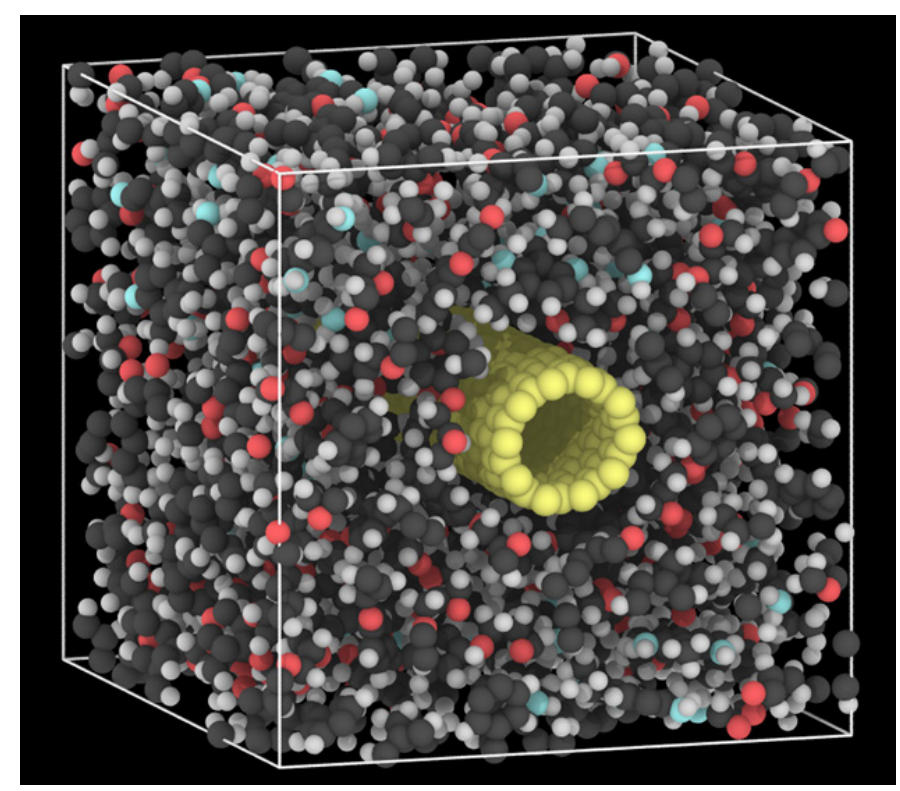

Figure 5-2. Representative CNT (yellow) and epoxy (CPK coloring) model.

\subsubsection{Interaction Energy}

To further understand the interfacial characteristics of the CNT/epoxy models, the interaction energy was calculated as,

$$
E_{\text {interaction }}=E_{\mathrm{CNT} / \text { ppoxy }}-E_{\mathrm{CNT}}-E_{\text {epoxy }}
$$

where is $E_{\mathrm{CNT} / \text { epoxy }}$ is the potential energy of the entire model and $E_{\mathrm{CNT}}$ and $E_{\text {epoxy }}$ are the potential energies of the CNT and epoxy, respectively. The CNT energy was determined by isolating the CNT at an instant and recalculating the potential energy without updating the atomic coordinates. Likewise, the epoxy energy was calculated by "deleting" the CNT. Thus, the interaction energy was calculated as a single-point property. For each model, the 
final interaction energy was obtained as by averaging the interaction energies of 250 "snapshots". The snapshots were obtained over the course of a 100 ps NPT simulation.

The interaction energies calculated for all $15 \mathrm{MD}$ models before and after curing are shown in Figure 5-3. It should be noted that a "more negative" interaction energy corresponds to a stronger CNT/epoxy interaction. Prior to curing, the Di, Tri, and Tetra models demonstrate average interaction energies of $-485,-484$, and $-491 \mathrm{kcal} / \mathrm{mol}$, respectively. The curing process results in a significant weakening of CNT-epoxy interaction which could be due to the rigidity of the polymer network formed. The unreacted monomers are expected to be more mobile and can more readily conform to the CNT. However, after curing, the monomeric units are constrained by the network, preventing the molecular groups from configuring well about the CNT. The average post-curing interaction energies for the Di, Tri, and Tetra models are equal to $-450,-447$, and -433 , respectively. The final Di and Tri models yield similar interaction energies. For the Tetra models, there is one anomalous sample with excellent interaction, but the majority of Tetra samples demonstrate relatively weak interaction.

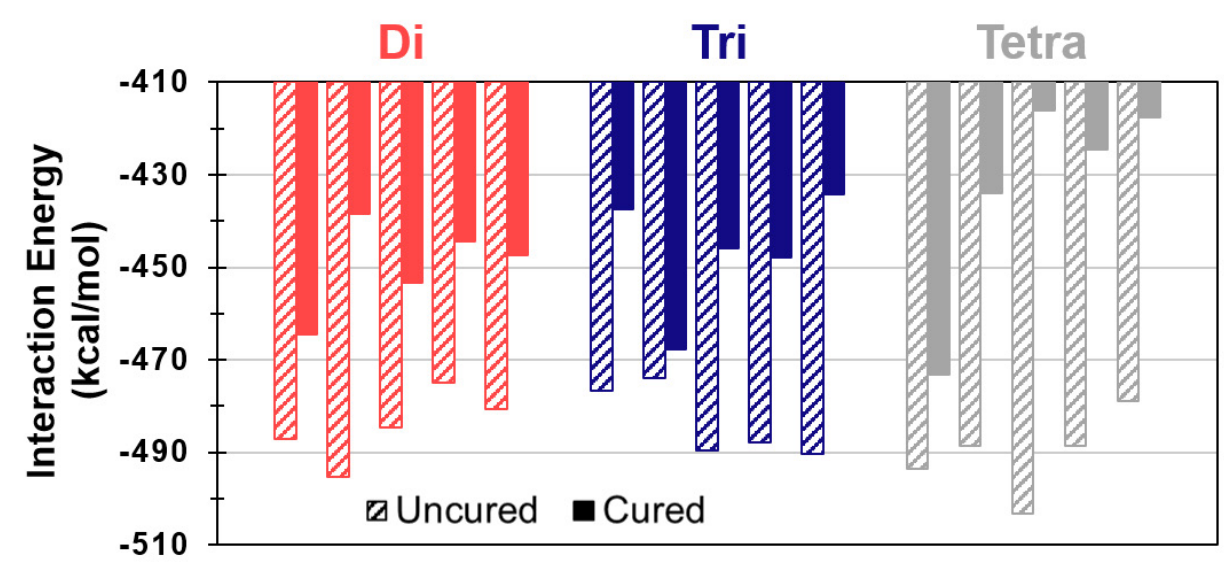

Figure 5-3. Interaction energies for all $15 \mathrm{MD}$ models

The interaction energies for each epoxy type were analyzed by elements. For example, the contribution due to the nitrogen atoms was calculated in the following manner: the potential energy of the nitrogen atoms in the absence of CNT was subtracted from the potential energy of the nitrogen atoms in the presence of CNT. This follows from Equation 
(5-1). The resulting interaction energy breakdown by element is displayed in Figure 5-4. Note that the sum of the energy terms do not add up to the values shown in Figure 5-3 since the CNT atoms are omitted. Figure 5-4 indicates the carbon, oxygen, and nitrogen atoms contribute favorably to the CNT/epoxy interaction. However, there is significant repulsion due to the hydrogen atoms, weakening interaction. The energy due to the favorable contributors $(\mathrm{C}, \mathrm{O}$, and $\mathrm{N})$ sum up to $-472,-552$, and $-570 \mathrm{kcal} / \mathrm{mol}$ for the Di, Tri, and Tetra models, respectively. Considering these atoms alone, it would appear that the Tetra model demonstrates the best interaction. However, for the Tetra model, the hydrogen repulsion is most detrimental, and the interaction energy contribution due to $\mathrm{H}$ is equal to $841 \mathrm{kcal} / \mathrm{mol}$. In contrast, the $\mathrm{H}$ energy contributions for the Di and Tri models are 659 and $762 \mathrm{kcal} / \mathrm{mol}$, respectively.

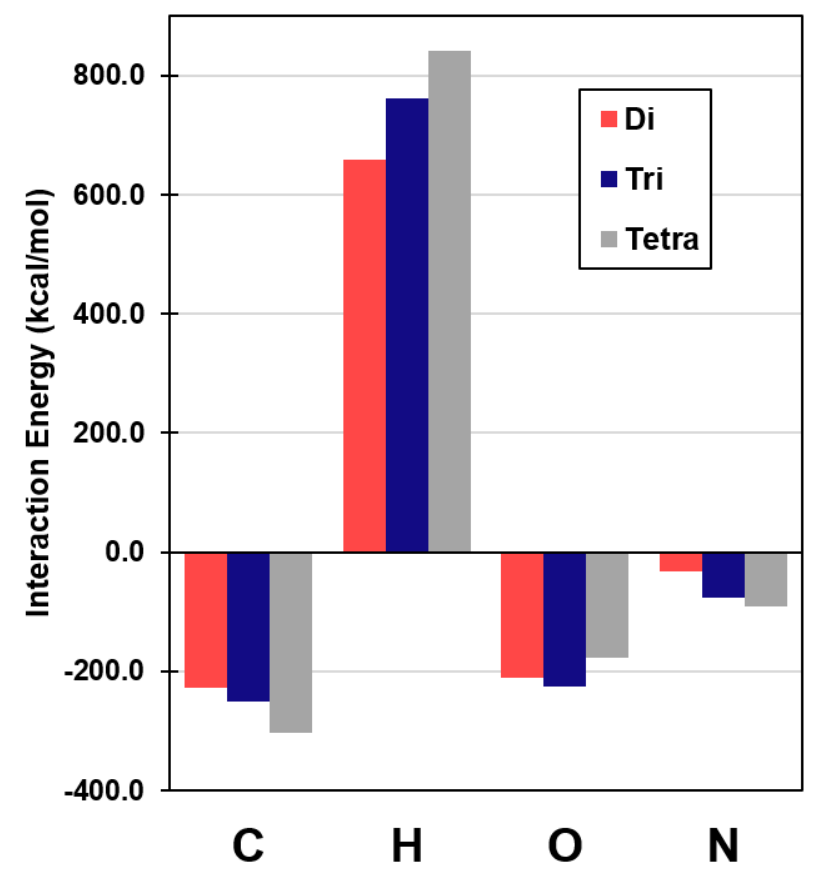

Figure 5-4. Contribution to interaction energy by epoxy elements

\subsubsection{Interphase Density}

To analyze the influence of the CNT on the neighboring epoxy, the MD model was partitioned using cylindrical shells centered about the CNT axis. The mass density of each 
cylindrical shell region was determined. For each model, 250 instants were considering spanning 100 ps of NPT simulation. These 250 "snapshots" were then averaged to obtain a density profile for the individual model.
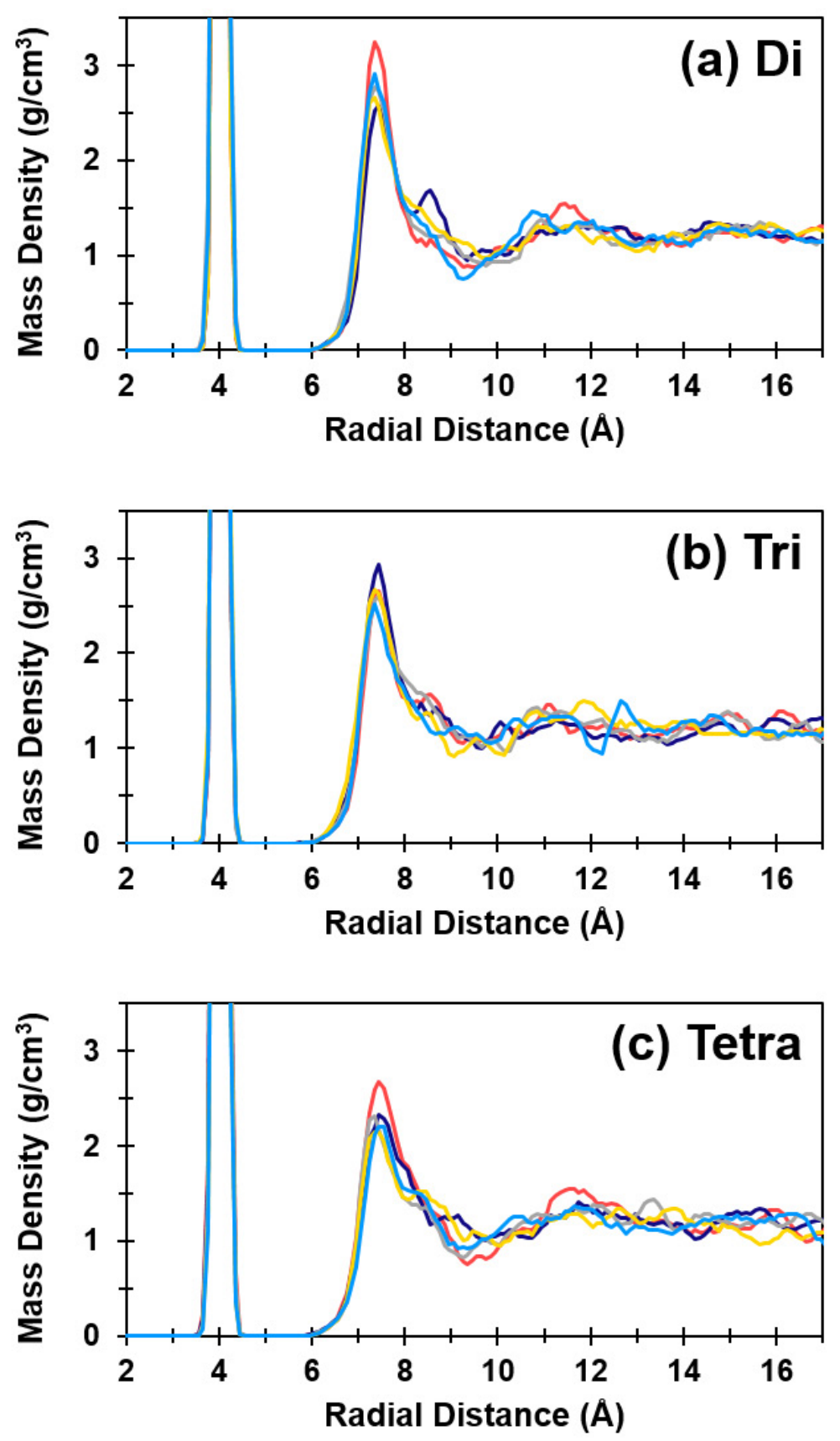

Figure 5-5. Radial distribution profiles for all samples of each epoxy type 
The radial density profiles of all Di- models are shown in Figure 5-5a. The spike in density at about $4 \AA$ is due to the CNT. At radial distances of about $6 \AA$ and greater, the region contains epoxy exclusively. From about 7-8.5 $\AA$, the local density exceeds that of the bulk epoxy $\left(1.2 \mathrm{~g} / \mathrm{cm}^{3}\right)$. Moving further away from the CNT, the epoxy density variations subside. Similar characteristics are observed for the Tri- and Tetra- models shown in Figure $5-5$ b-c.
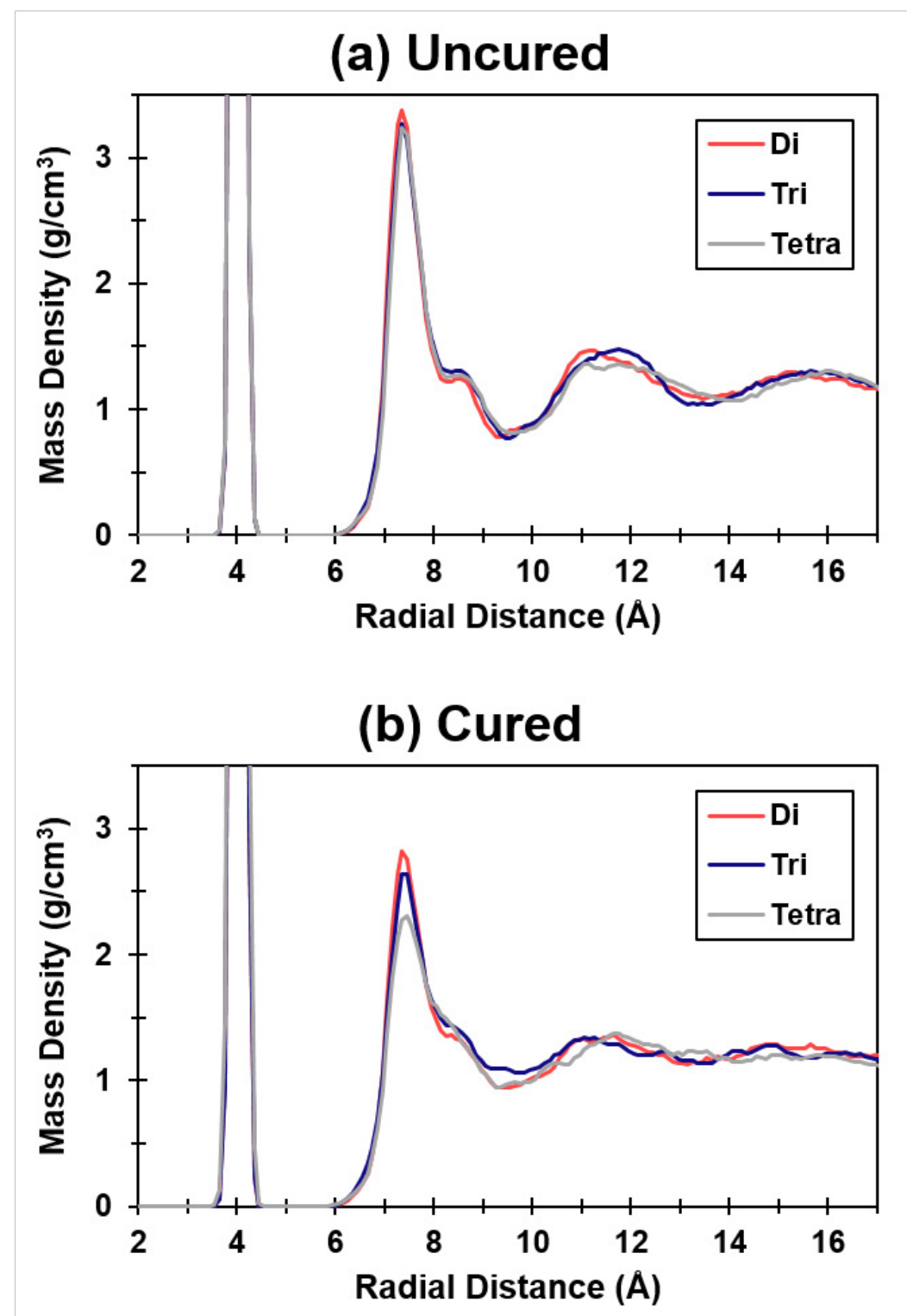

Figure 5-6. Average radial density profiles for (a) uncured and (b) cured models of each epoxy type. Average taken over five samples. 
Figure 5-6 a-b exhibit the density profiles of equilibrated uncured models and cured models, respectively. Comparing the uncured and cured models, a notable difference is the peak density of the matrix region. For the uncured epoxies, the average peak densities fall within 3.2-3.4 $\mathrm{g} / \mathrm{cm}^{3}$. However, crosslinking causes the density peaks to drop, which confirms that the crosslinked network constrains its constituents from packing ideally about the CNT. After curing, the resulting profiles become more distinct. The cured Di model obtains the highest average peak density, $2.83 \mathrm{~g} / \mathrm{cm}^{3}$. Whereas, the cured Tri and Tetra systems provide average peak densities of 2.67 and $2.34 \mathrm{~g} / \mathrm{cm}^{3}$, respectively. Thus, the difunctional resin epoxy appears to best conform to the CNT after crosslinking.

To further analyze the density peaks shown here, the densities profiles are plotted for each element in the epoxy. Figure 5-7 shows the carbon, hydrogen, oxygen, and nitrogen contributions for the Di epoxy. It is clear that the carbon atoms primarily contribute to the overall density peak. Density peaks are observed for the carbon, hydrogen, and oxygen atoms, but there is no discernable peak for the nitrogen atoms. The hydrogen peak appears at about $6.7 \AA$ which is closer than the carbon or oxygen peaks. However, the hydrogen peak is not conspicuous in the overall density profile due to the low atomic mass of hydrogen.

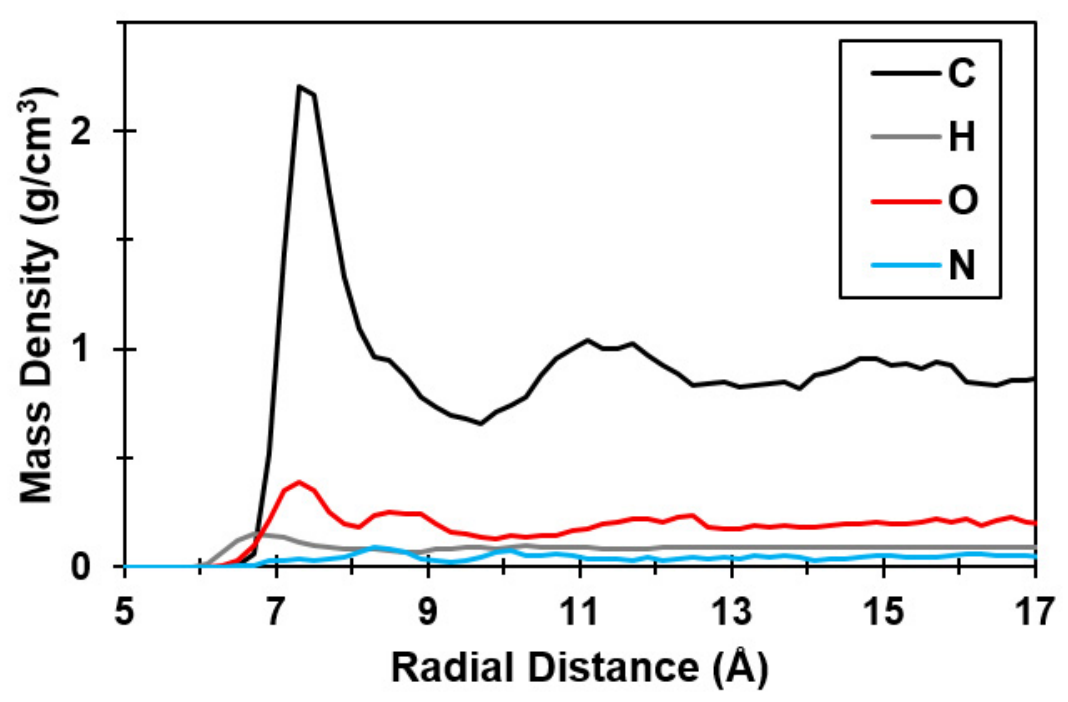

Figure 5-7. Density profile by element for Di epoxy 
It has been conjectured that density profiles, such as shown here, provide information concerning the nanoparticle/matrix interaction. To demonstrate the relationship between the density peak of the epoxy region and CNT/epoxy interaction energy, the two characteristics are plotted in Figure 5-8, and all 15 CNT/epoxy models before and after crosslinking are shown. Going from left to right on the interaction energy scale indicates weakening interaction which is accompanied by a decreasing density peak. Strong correlation is observed, and in general, the models with strong interaction resulted in high matrix density peaks. Thus, we confirm that the matrix density profile does indeed provide valuable information concerning the CNT-epoxy interaction.

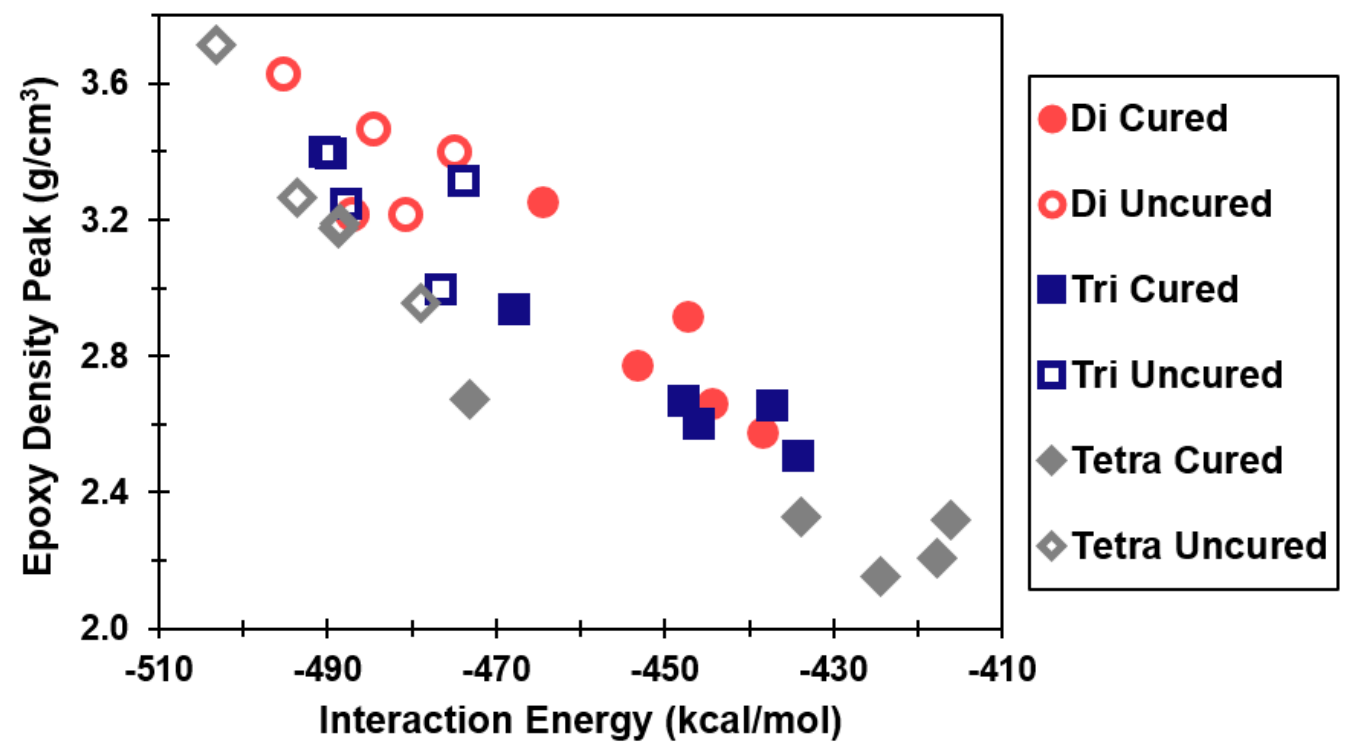

Figure 5-8. Relationship between epoxy density peak and CNT/epoxy interaction energy for all models

The implications of the network structural impact on nanoparticle-matrix adhesion are significant. In Chapter 3, the pure Di epoxy (without CNT) was shown to be the most compliant of the three studied here; whereas, the pure Tetra epoxy is the stiffest [18]. The soft epoxy is demonstrated to more readily conform to the CNT and pack closely around it (as manifested in the density profiles) than the stiff epoxy, resulting in greater adhesion. For a highly stiff nanocomposite, not only is strong interaction required, but also high bulk stiffness is desired. However, these properties are conflicting, that is, promoting the bulk epoxy stiffness adversely affects the CNT-epoxy interaction. The CNT/epoxy models 
developed here elucidate an underlying cause of the conflict. The amount of covalent bonding between monomers and the amount of crosslinks required to form a mechanically stiff epoxy also reduces the conformability of the network and consequently reduces its ability to closely bind to nanoparticles.

\subsubsection{Orientation of Benzene Rings}

The orientation of benzene rings with respect to the CNT was also analyzed in the MD models. Each benzene ring was identified and fit with a plane. The general equation for a plane in $3 \mathrm{~d}$ space is given by,

$$
a x+b y+c z+d=0
$$

The plane of a benzene ring was compared to a plane tangent to the CNT surface. Given these two planes, the dihedral angle between planes can be found in following manner:

$$
\cos (\theta)=\frac{a_{1} a_{2}+b_{1} b_{2}+c_{1} c_{2}}{\sqrt{a_{1}^{2}+b_{1}^{2}+c_{1}^{2}} \sqrt{a_{2}^{2}+b_{2}^{2}+c_{2}^{2}}}
$$

where the two planes are distinguished by the coefficient subscripts.

An example plot showing the orientation of benzene rings as a function of radial distance is shown in Figure 5-9. Note that when the ring orientation is $0^{\circ}$, the benzene ring is parallel to the CNT surface. The orientation of rings in the interphase region (denoted by a small radial distance) was observed to identify if a majority of rings aligned with the CNT surface. However, no clear pattern was identified with the MD models. While it is generally observed that parallel aromatic rings provide for good non-bonded interaction, there are a number of factors that could inhibit such alignment in our models. While ReaxFF is parameterized using quantum level calculations, all effects of electronic orbitals are absorbed in a simplified atomic-distanced-based function. It is possible that since MD does not explicitly model electronic orbitals, $\pi-\pi$ stacking is not well-captured. Also, the polymer network structure may not allow the units to easily conform to the CNT to achieve a high 
percentage of aligned benzene rings, and the high curvature of the CNT modeled may prevent ring alignment as well.

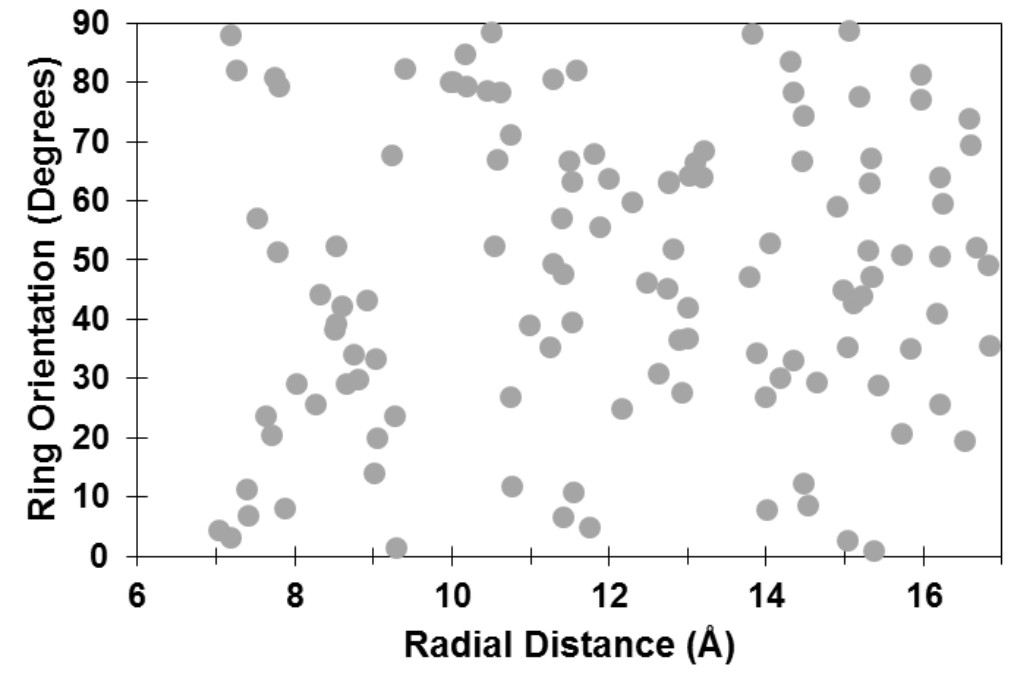

Figure 5-9. Orientation of benzene rings with respect to CNT surface for a single CNT/epoxy model

\subsubsection{Mechanical Deformation}

Each crosslinked, equilibrated model was subjected to uniaxial tension and shear deformation simulations. Three tension simulations were conducted per model: tension in the $\mathrm{x}-, \mathrm{y}-$, and $\mathrm{z}$ - directions. The simulation box was gradually deformed up to $10 \%$ engineering strain over 500 ps resulting in a strain rate of $2 \mathrm{e} 8 \mathrm{~s}^{-1}$. The NPT ensemble was implemented to allow for Poisson's contractions. A Nose-Hoover barostat was set to maintain 1 atm of pressure on the contracting sides. Additionally, three simple shear simulations were performed for each model: shear in the xy-, xz-, and yz- planes. The NVT ensemble was selected, and the models were deformed up to $5 \%$ shear strain over $250 \mathrm{ps}$ ( $2 \mathrm{e} 8 \mathrm{~s}^{-1}$ strain rate). All deformation simulations were carried out at $300 \mathrm{~K}$ with a timestep of 0.1 fs.

From each deformation simulation, the stress-strain data was fit with a linear regression model for strains up to about 3\% strain. Figure 5-10 provides an example stress-strain plot obtained from uniaxial tension transverse to the CNT. The Poisson's ratios were also 
obtained from each tension simulation. The lateral strains were plotted with respect to the tensile strain and fit with a linear regression model for tensile strains up to $3.5 \%$.

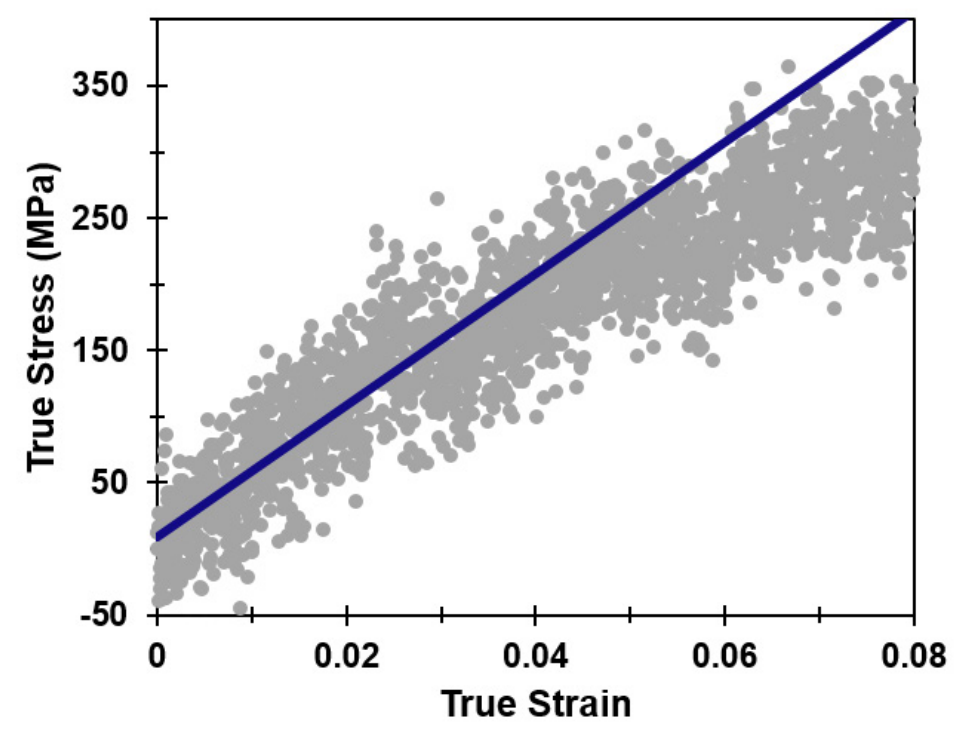

Figure 5-10. Representative stress-strain curve with linear fit

The mechanical properties obtained from MD simulation are given in Table 5-3. The properties listed are the axial and transverse Young's moduli $\left(E_{A}\right.$ and $\left.E_{T}\right)$, axial and transverse Poisson's ratio $\left(v_{A}\right.$ and $\left.v_{T}\right)$, and axial and transverse shear moduli $\left(G_{A}\right.$ and $\left.G_{T}\right)$. For all Young's moduli and shear moduli, the Di model provides the least average value and Tri model provides the greatest average value. Even though there is considerable variation in values for some moduli, the consistency in the order Tri $>$ Tetra $>$ Di supports making such a distinction. There are statistically significant differentiations that can be asserted in support of this ordering. Most apparently, the Tri model resulted in an axial Young's modulus of 76.0 GPa, clearly outperforming the Di (71.2 GPa) and Tetra (72.9 GPa) models. For the transverse Young's modulus results, the Di model yields a value of $3.88 \mathrm{GPa}$ which is undoubtedly the least stiff of the three epoxies. The $E_{T}$ values for the Tri and Tetra are 5.40 and 5.09 GPa, respectively, but we cannot show statistical significance here when considering the standard deviations. Either additional models or larger models would be required to fully assert that the Tri epoxy provides the greatest values for all stiffness properties. However, the superior stiffness of the Tri model can be argued by considering 
the pure epoxy moduli and the strength of the CNT-epoxy interaction. First, recall that the pure Di epoxy is the least stiff and the pure Tetra epoxy is the stiffest. The modulus of the pure Tri epoxy falls in between the Di and Tetra epoxies. Secondly, the Tetra epoxy interacts weakly with CNT in comparison with the Di and Tri epoxies as demonstrated in the previous section. Thus, the weakness of the Di nanocomposite model is the soft bulk matrix, and the weakness of the Tetra nanocomposite model is the relatively poor CNTepoxy interaction. We suggest that the Tri nanocomposite model surpasses the Di and Tetra models because it possesses both good bulk stiffness and good interaction.

Table 5-3. Elastic moduli from MD simulation

\begin{tabular}{|c|c|c|c|c|c|c|}
\hline EPOXY & $\begin{array}{c}\boldsymbol{E}_{\boldsymbol{A}} \\
\mathbf{G P a}\end{array}$ & $\begin{array}{c}\boldsymbol{E}_{\boldsymbol{T}} \\
\mathbf{G P a}\end{array}$ & $\boldsymbol{v}_{\boldsymbol{A}}$ & $\boldsymbol{v}_{\boldsymbol{T}}$ & $\begin{array}{c}\boldsymbol{G}_{\boldsymbol{A}} \\
\mathbf{G P a}\end{array}$ & $\begin{array}{c}\boldsymbol{G}_{\boldsymbol{T}} \\
\mathbf{G P a}\end{array}$ \\
\hline $\mathrm{Di}-$ & $71.2 \pm 1.8$ & $3.88 \pm 0.78$ & $0.39 \pm 0.18$ & $0.61 \pm 0.08$ & $1.19 \pm 0.25$ & $1.63 \pm 0.18$ \\
Tri- & $76.0 \pm 0.5$ & $5.40 \pm 0.84$ & $0.33 \pm 0.09$ & $0.54 \pm 0.06$ & $1.60 \pm 0.46$ & $2.19 \pm 0.32$ \\
Tetra- & $72.9 \pm 0.8$ & $5.09 \pm 0.77$ & $0.35 \pm 0.12$ & $0.56 \pm 0.09$ & $1.50 \pm 0.46$ & $1.79 \pm 0.32$ \\
\hline
\end{tabular}

\subsection{Micromechanics}

While the influence of nanoparticle-matrix interactions is observed in the MD mechanical response, it remains be seen what effect these non-bonded interactions have on the macroscale properties. Will differences in the CNT/epoxy interfacial strengths perform a major role? Will the excellent mechanical properties of the Tri MD model translate into an overall superior nanocomposite? The answer demands a multiscale modeling approach to predict the effective moduli of realistic composites based on the MD modeling results. Hadden et al. successfully demonstrated a combined MD-micromechanics approach to predict the effective properties of epoxy reinforced with graphene nanoplatelets (GNPs) and CF/GNP/epoxy hybrid composites [100]. The method employed in this study is derived from the the work of Hadden et al. Four micromechanics steps are involved, which are presented in Figure 5-11 and will be elaborated on in this section. 


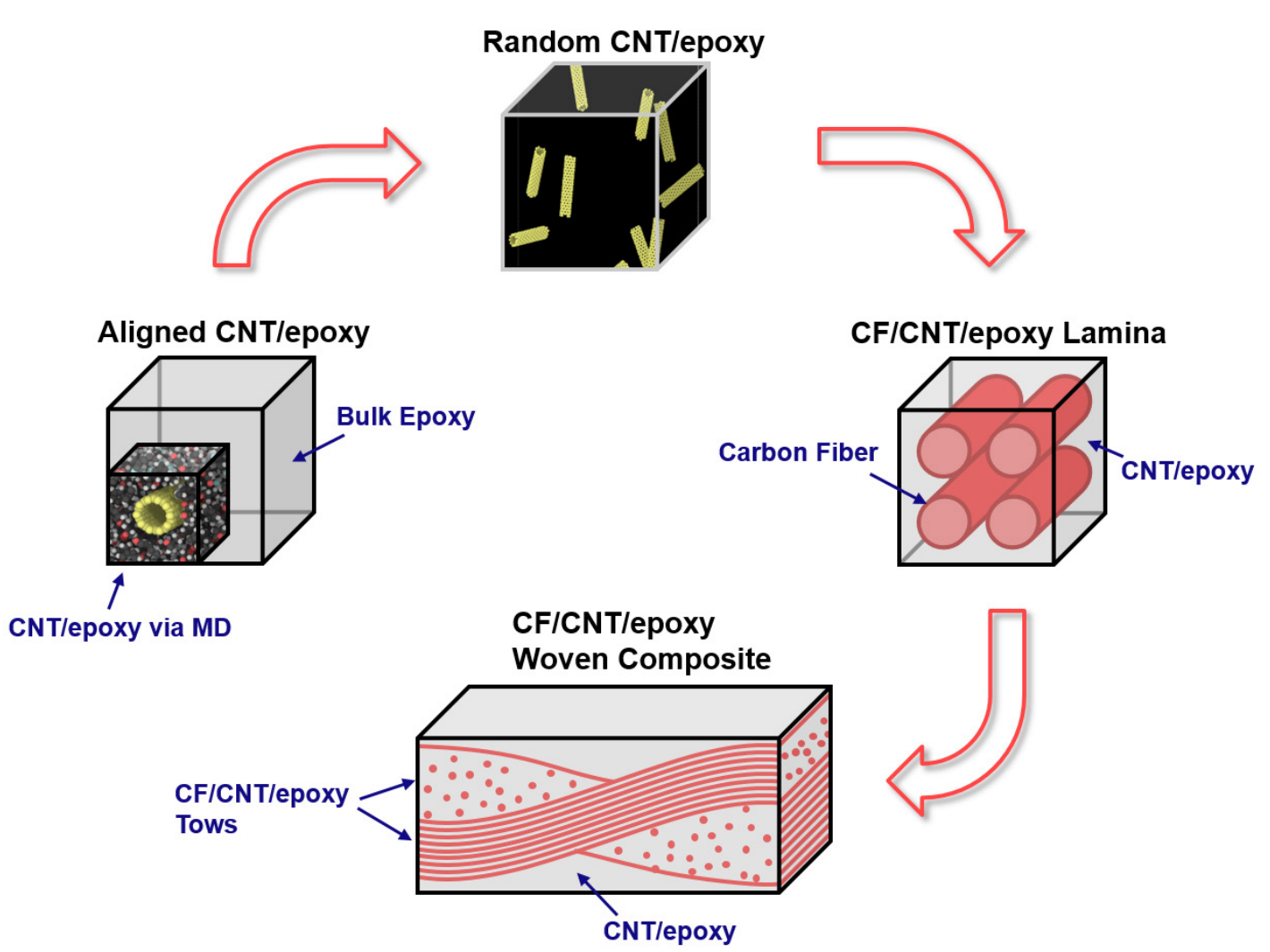

Figure 5-11. Multiscale Modeling Scheme

\subsubsection{Randomly Dispersed CNT in Epoxy}

First, the properties of an aligned CNT/epoxy system were obtained for various epoxy types, CNT wt\%, and CNT aspect ratios. Using MAC/GMC [101], a micromechanics software, the CNT concentration can be scaled without running additional MD simulations. A 2D representative unit cell (RUC) was employed for infinitely long CNT. For finite CNTs, a 3D RUC shown in Figure 5-12 was used. The properties of the MD CNT/epoxy model (Table 5-3) were input into subcell MD (see Figure 5-12), and the remaining subcells were assigned the bulk epoxy properties shown in Table 5-4. The aspect ratio $l / w$ of subcell $M D$ was adjusted to reflect the aspect ratio of CNT. If $w$ is chosen to be consistent with the MD simulation box (about $35 \AA$ in this case), then the subcell length $l$ will directly determine the CNT aspect ratio $a_{C N T}$ in the following manner: 


$$
a_{C N T}=\frac{l}{d_{C N T}}
$$

where $d_{C N T}$ is the CNT diameter.

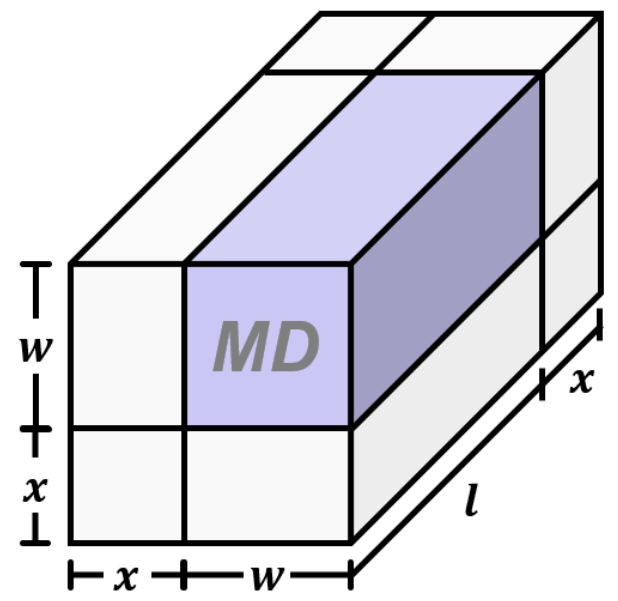

Figure 5-12. 3D RUC for finite CNT aspect ratios

To consider different amounts of CNT, the volumetric ratio of subcell $M D$ to the entire RUC was adjusted. The concentration of CNT will be expressed in $\mathrm{wt} \%$ as opposed to volume fraction to avoid making assumptions concerning the volume of a nanotube. The overall $\mathrm{wt} \%$ of CNT $w_{C N T / R U C}$ can be determined by,

$$
w_{C N T / R U C}=w_{C N T / M D} * w_{M D / R U C}
$$

where $w_{C N T / M D}$ is the CNT wt $\%$ of the MD model (Table 5-2) and $w_{M D / R U C}$ is the $\mathrm{wt} \%$ of the MD model to the entire RUC. Moreover, $w_{M D / R U C}$ can be found from the density of the MD model $\rho_{M D}$, the bulk epoxy density $\rho_{B}$, and the volume fractions of the subcell $M D$ and bulk subcells to the RUC, $v_{M D / R U C}$ and $v_{B / R U C}$, respectively:

$$
w_{M D / R U C}=\frac{\rho_{M D} v_{M D / R U C}}{\rho_{M D} v_{M D / R U C}+\rho_{B} v_{B / R U C}}
$$


The densities of the MD models and bulk epoxy are given in Table 5-2 and Table 5-4. Thus, from Equations (5-5) and (5-6), the overall CNT wt\% could be calculated given the RUC geometry.

The properties of aligned CNT/epoxy were integrated over all directions to predict the effective properties of randomly dispersed CNT in epoxy [100, 102]. While the aligned material is transversely isotropic, the final nanocomposite is isotropic. The properties for $\mathrm{CNT} /$ epoxy obtained in this step were re-input into MAC/GMC to examine two hybrid composites as described in the following sections. Note that this method treats the CNT as being perfectly dispersed.

Table 5-4. Properties used for bulk epoxy

\begin{tabular}{|c|c|c|c|}
\hline EPOXY & $\begin{array}{c}\boldsymbol{E} \\
\text { GPa }\end{array}$ & $\boldsymbol{v}$ & $\begin{array}{c}\text { DENSITY } \\
\mathbf{g} / \mathbf{c m}^{\mathbf{3}}\end{array}$ \\
\hline Di- & 3.51 & 0.37 & 1.222 \\
Tri- & 4.94 & 0.37 & 1.235 \\
Tetra- & 5.30 & 0.35 & 1.221 \\
\hline
\end{tabular}

\subsubsection{CF/CNT/epoxy Lamina}

The effective properties of a $\mathrm{CF} / \mathrm{CNT} / \mathrm{epoxy}$ hybrid composite were calculated via MAC/GMC. Here we considered unidirectional CF (as in a composite lamina) in a CNTenhanced matrix. The properties of random CNT/epoxy as determined in the previous micromechanics step was input as the matrix, and the AS4 carbon fiber properties shown in Table 5-5 were selected. The matrix and fiber properties were assigned to a 2D RUC consisting of 676 subcells to approximate a circular fiber. This RUC is available in MAC/GMC as designated by the architecture ID number 13. The fibers were arranged in a square packing array. In this manner, the properties of a CF/CNT/epoxy lamina were obtained for various epoxy types, CNT wt\% (with respect to the matrix), CNT aspect ratio, and $\mathrm{CF}$ volume fraction.

Table 5-5. AS4 carbon fiber properties

\begin{tabular}{|c|c|c|c|c|}
\hline $\begin{array}{c}\boldsymbol{E}_{\boldsymbol{A}} \\
\mathbf{G P a}\end{array}$ & $\begin{array}{c}\boldsymbol{E}_{\boldsymbol{T}} \\
\mathbf{G P a}\end{array}$ & $\boldsymbol{v}_{\boldsymbol{A}}$ & $\boldsymbol{v}_{\boldsymbol{T}}$ & $\begin{array}{c}\boldsymbol{G}_{\boldsymbol{A}} \\
\mathbf{G P a}\end{array}$ \\
\hline 235 & 15 & 0.20 & 0.07 & 27 \\
\hline
\end{tabular}




\subsubsection{CF/CNT/Epoxy Woven Composite}

The woven hybrid composite was modeled using a two-step homogenization procedure outlined by Bednarcyk and Arnold [103]. The matrix and fiber properties in Table 5-4 and Table 5-5 were input in MAC/GMC using the MSGMC command. The geometric properties of the weave were constant for all calculations. The overall geometry of the plain weave is shown in Figure 5-13. The tow width $a$ and tow spacing $g$ were 1.0 and 0.95, respectively, and the height of the composite $H$ was set to 0.78 . These parameters completely define the geometry of the RUC. The inclination angle $\theta$ of the tows was determined as,

$$
\theta=\tan ^{-1}\left(\frac{H}{2 g}\right)
$$

The tow fiber volume fraction $\left(v_{f}\right)_{\text {tow }}$ was varied to obtain a range of overall fiber volume fractions $\left(v_{f}\right)_{\text {comp }}$ which was calculated from in the following manner:

$$
\left(v_{f}\right)_{\text {comp }}=\frac{a}{a+g}\left(v_{f}\right)_{\text {tow }}
$$

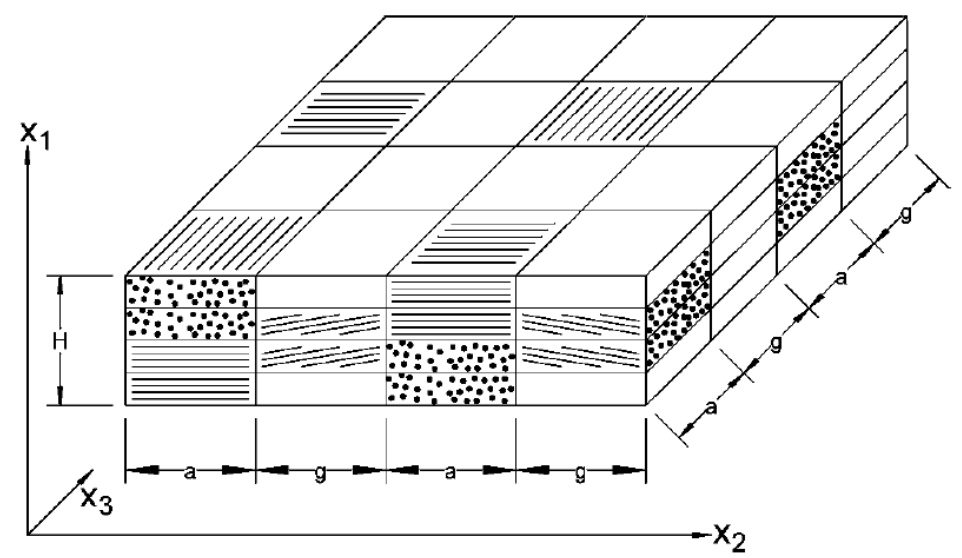

Figure 5-13. MAC/GMC RUC for plain weave fabric [103]. Reproduced with permission of the American Institute of Aeronautics and Astronautics. (See Appendix B for copyright agreement) 


\subsection{Multiscale Modeling Predictions}

\subsubsection{Randomly Dispersed CNT in Epoxy}

For the Di epoxy, the predicted stiffness for randomly dispersed CNT/epoxy is shown in Figure 5-14. The normalized modulus presented is given by dividing the nanocomposite modulus by the pure matrix modulus. Results are shown for various CNT concentrations and CNT aspect ratios. The reinforcing effect of CNT is shown to increase with increasing aspect ratio but plateaus for CNT aspect ratio greater than 10,000. For example, the infinitely long CNT demonstrates nearly the same effect as CNT with an aspect ratio of 10,000. Comparing the CNT aspect ratios of 100 and 1,000, a more detectable difference is observed. For $1 \mathrm{wt} \% \mathrm{CNT}$, the normalized moduli for 100 and 1,000 CNT aspect ratios are 1.10 and 1.23, respectively. This amounts to a difference in overall Young's modulus of about 0.3-0.4 GPa.

The predictions obtained in this study are compared with experimental values in the literature. Using the same epoxy resin and hardener, Sun et al. obtained a normalized modulus of 1.18 for $1 \mathrm{wt} \%$ unfunctionalized SWCNT [104]. It was reported that the CNTs supplied generally possessed aspect ratios above 1000. Our multiscale model predicts a normalized modulus of 1.23 for 1000 aspect ratio CNT which is slightly above the experimental value. We should note that, by SEM, Sun et al. observed agglomeration in the specimen containing pristine CNT. However, our model treats the CNT as being perfectly dispersed. Wang et al. (2006) observed a 3.2\% improvement in storage modulus due to unfunctionalized SWCNT reinforcement (BuckyPearls from Carbon Nanotechnologies Inc.) in the Di epoxy system [105]. BuckyPearls are reported to have an average aspect ratio of about 500 [106]. Our multiscale model predicts a higher modulus compared to the data point from Wang et al. Again, we conjecture that agglomeration in the experimental samples is the likely cause, lowering the modulus to be nearly equivalent to the model of welldispersed CNT with aspect ratio of 100. In a later study, Wang et al. (2009) obtained a higher normalized modulus, 1.15 , for $0.5 \mathrm{wt} \%$ SWCNT in the Di epoxy [107]. In this case, the aspect ratio of the SWCNTs was not reported. For further reference, the results from 
Gojny et al. are included in which DGEBA, a difunctional resin similar to our Di epoxy, is used [7]. The SWCNTs were reported to have an average diameter of less than $2 \mathrm{~nm}$ and lengths on the order of micrometers. The average CNT aspect ratio then is likely greater than 1000 . The data points obtained by Gojny et al. closely follow the high aspect ratio models.

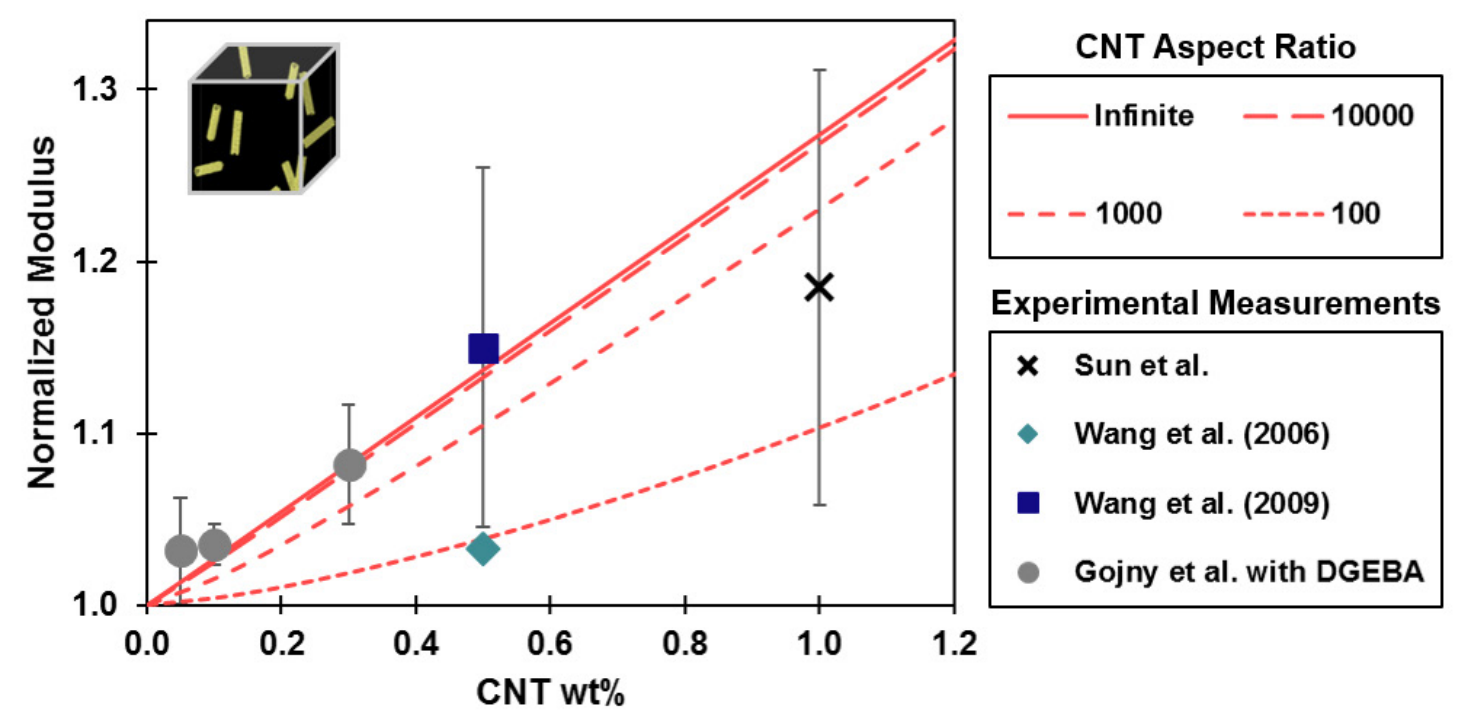

Figure 5-14. Normalized modulus for random CNT/epoxy using the Di epoxy (BFGDE/DETDA) as predicted by our multiscale model. Results are shown for various CNT concentration and CNT aspect ratios and compared with experimental literature values [7, 104, 105, 107]

The effective Young's moduli of random, infinite CNT in epoxy is shown in Figure 5-15. Both the Tri and Tetra epoxies demonstrate excellent stiffness for all CNT concentrations and aspect ratios. The nanoparticle-matrix interaction and load transfer characteristics can be inferred from the increase in the modulus relative to the bulk matrix. For example, we see that the good interaction of the Tri MD model allows the CNT/Tri nanocomposite to surpass the CNT/Tetra system for high CNT wt\%. (For 5 wt $\%$ of infinite CNTs, the predicted moduli for the Tri and Tetra models are 9.73 and $9.60 \mathrm{GPa}$, respectively. Similarly, for $5 \mathrm{wt} \%$ of CNTs with aspect ratio of 1000 , the predicted moduli for the Tri and Tetra models are 9.60 and $9.48 \mathrm{GPa}$.) For $5 \mathrm{wt} \%$ infinite CNT, the Di and Tri epoxy moduli both were improved by about $4.8 \mathrm{GPa}$. However, the Tetra epoxy was 
improved by about $4.3 \mathrm{GPa}$. Since the bulk Tetra epoxy is the stiffest out of the three studied, this matches with experiment observations that stiffer matrices are not as well improved by CNT as softer epoxies [8-12].

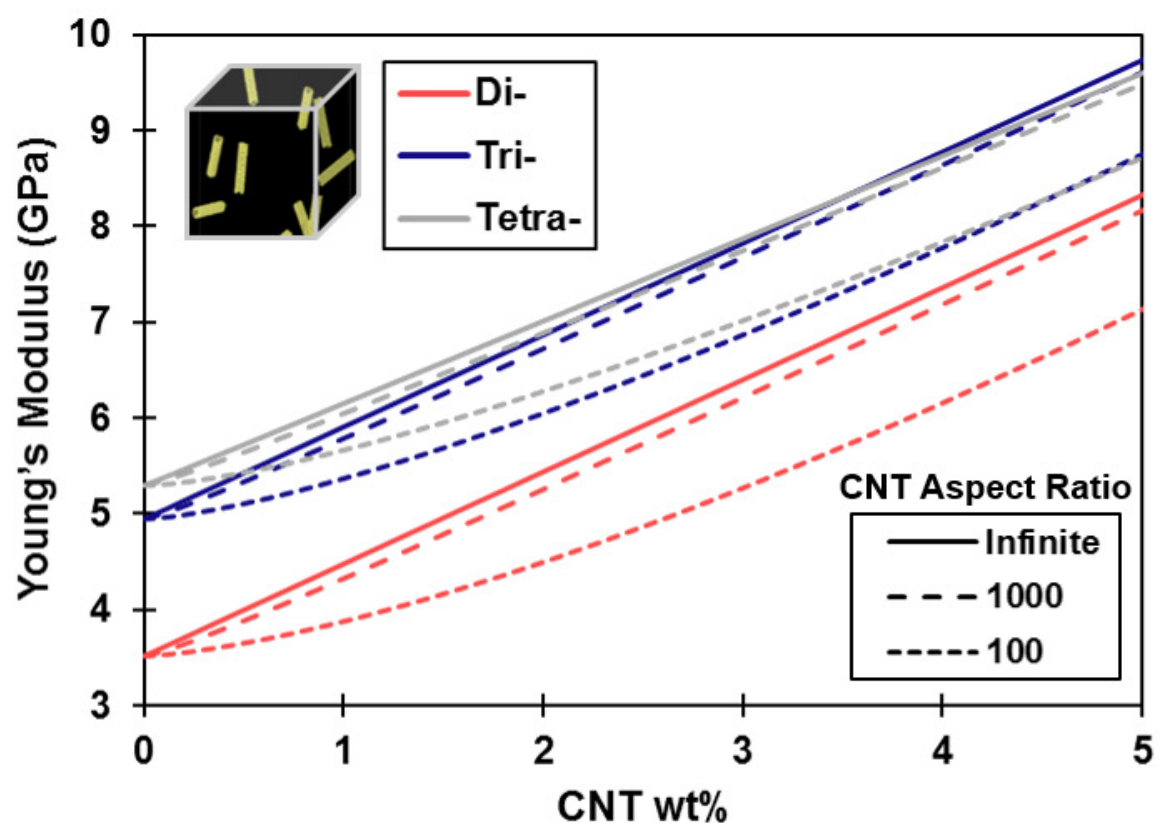

Figure 5-15. Effective Young's Modulus of random CNT/epoxy for various epoxy types and CNT aspect ratios

While the effect of the nanoparticle-matrix interaction can be observed in Figure 5-15, it is not the most significant factor within the range of CNT concentrations shown. Even though the Di epoxy demonstrates good nanoparticle-matrix load transfer, the CNT/Di nanocomposite remains the most compliant (just as the bulk Di epoxy) and cannot supersede CNT/Tetra at such CNT concentrations. Additionally, the Tri epoxy only slightly supersedes the Tetra epoxy. Thus, for limited CNT loadings, no distinction in non-bonded CNT-epoxy interaction resulted in any dramatic improvement with respect to the other epoxies. While the CNT-epoxy interaction does indeed contribute, it appears to be the secondary factor. Rather, the bulk matrix stiffness appears to be the dominant factor when comparing the overall nanocomposite stiffness between epoxies for CNT concentrations up to $5 \mathrm{wt} \%$.

We can also consider much higher CNT loadings, such as those obtained for CNT arrays infused with epoxy. For example, Bradford et al. produced CNT/epoxy composites (using the Di epoxy) with $32 \mathrm{wt} \%$ of CNTs aligned by shear pressing [108]. At such CNT 
loadings, the interaction characteristics become more critical, and the advantages of Tri epoxy over the Tetra epoxy are expected to become more pronounced. Additionally, the Di epoxy is predicted to surpass the Tetra. If we extrapolate the Young's modulus trend for infinite CNT, the Di model will supersede the Tetra for CNT loadings above $17 \mathrm{wt} \%$.

Notwithstanding the differences discussed above, all epoxy types are predicted to be greatly enhanced by well-dispersed CNT. While the Tetra epoxy is noted to possess the weakest nanoparticle-matrix interaction, $5 \mathrm{wt} \%$ infinite $\mathrm{CNT}$ results in a predicted increase in Young's modulus of $81 \%$. Therefore, we do not expect the weaker CNT-Tetra interface to altogether inhibit reinforcement.

\subsubsection{CF/CNT/epoxy Lamina}

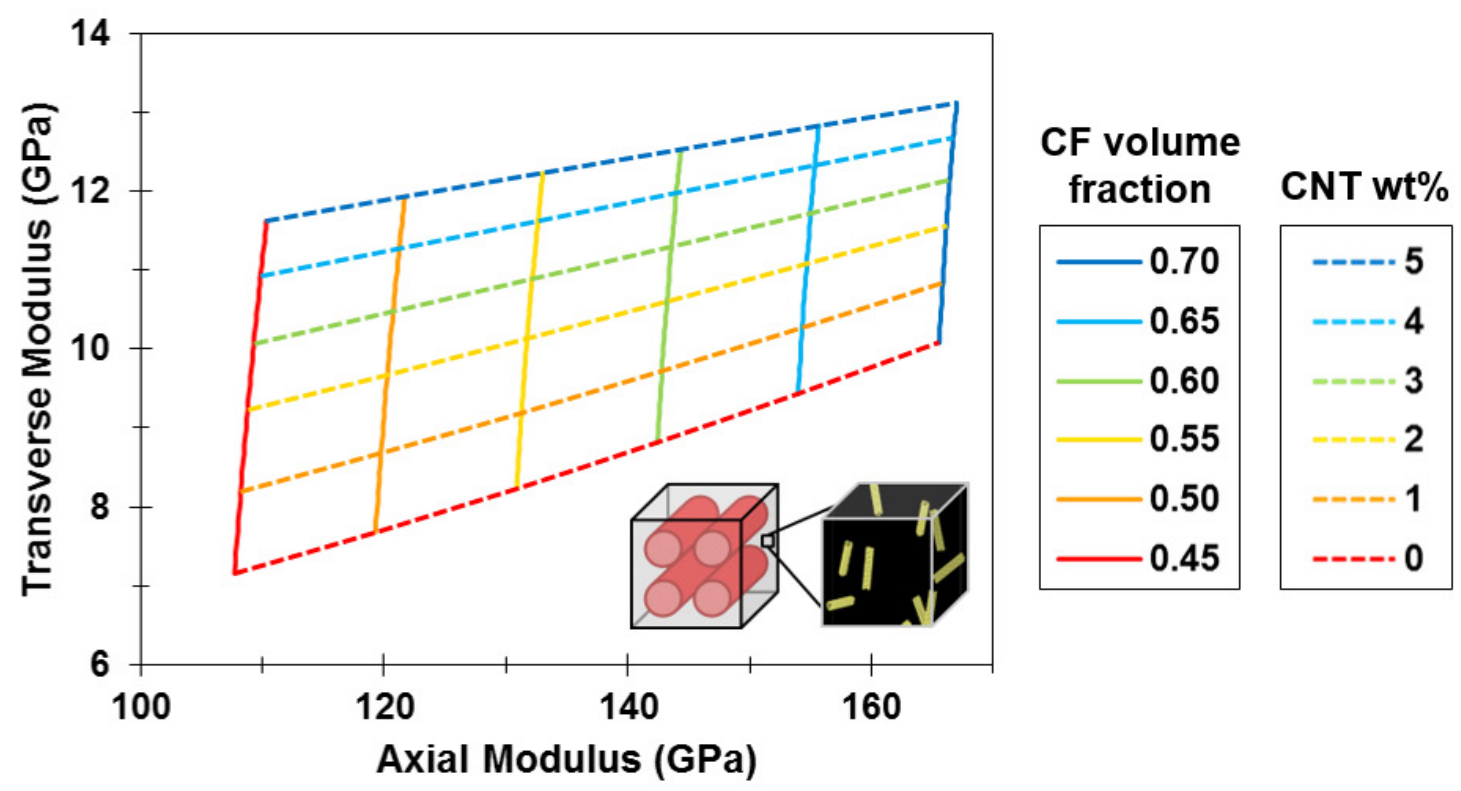

Figure 5-16. Predicted axial and transverse moduli for CF/CNT/epoxy with the Di epoxy. Various fiber volume fractions and CNT wt $\%$ are shown. CNT is treated as having an infinite aspect ratio.

Figure 5-16 shows a CF/CNT/epoxy (for the Di epoxy) design map for optimizing the axial and transverse moduli by controlling the $\mathrm{CF}$ and $\mathrm{CNT}$ concentrations. Note that the $\mathrm{CNT}$ is treated as having an infinite aspect ratio. The results demonstrate that both the CF and CNT have a major impact on the elastic properties of the hybrid composite. As 
expected, including CNT primarily increases the transverse modulus, as it is a matrixdominated property.

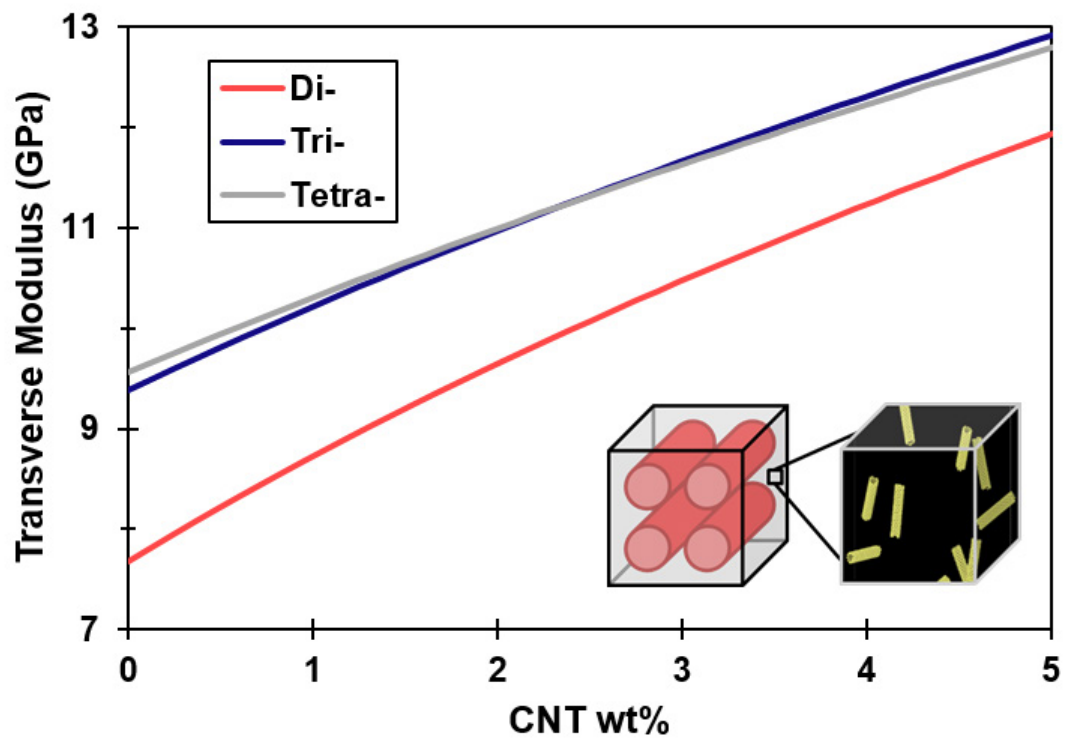

Figure 5-17. Effective transverse modulus for CF/CNT/epoxy hybrid laminas for various epoxy types. Volume fraction of $\mathrm{CF}$ is 0.60 .

The influence of different epoxy types on the transverse modulus of a $\mathrm{CF} / \mathrm{CNT} /$ epoxy lamina is shown in Figure 5-17. Similar to the CNT/epoxy results, the Tri and Tetra epoxies provide for the stiffest hybrid composites for all CNT concentrations. The results shown in Figure 5-17 were obtained for a $\mathrm{CF}$ volume fraction of 0.60 . The $\mathrm{CF} / \mathrm{CNT} / \mathrm{Tri}$ and $\mathrm{CF} / \mathrm{CNT} /$ Tetra models outperformed the $\mathrm{CF} / \mathrm{CNT} / \mathrm{Di}$ for other $\mathrm{CF}$ loadings as well. While the axial modulus is dominated by the CF and only minorly affected by CNT, we observed the axial modulus was slightly greater (about $1 \mathrm{GPa}$ ) for the Tri and Tetra epoxies when compared to the Di epoxy for various CNT concentrations.

\subsubsection{CF/CNT/Epoxy Woven Composite}

Figure 5-18 and Figure 5-19 show the predicted in-plane moduli for various epoxy types and CNT concentrations. These figures contain predictions for both 0.30 and 0.40 overall fiber volume fractions. Here the CNT is treated as having an infinite aspect ratio. As 
observed for the CNT/epoxy nanocomposite, the Tri and Tetra epoxies provide for excellent hybrid fabric stiffness for all CNT concentrations; whereas, the hybrid composites with the Di epoxy are the most compliant. This is consistent for all CF volume fractions. Our multiscale model indicates that the concentration of CNT has a significant effect on the both the in-plane Young's modulus and in-plane shear modulus. Consider the woven CF/CNT/Di composite with $40 \mathrm{vol} \%$ carbon fiber. Adding $5 \mathrm{wt} \% \mathrm{CNT}$ to the matrix results in a $42 \%$ increase of the in-plane Young's modulus and 111\% increase of the in-plane shear modulus when compared with no CNT reinforcement.

A design for optimizing the in-plane moduli of a $\mathrm{CF} / \mathrm{CNT} / \mathrm{Di}$ woven composite is shown in Figure 5-20. It is observed that increasing the CNT concentration results in a more significant impact on the in-plane shear modulus than increasing the amount of CF.

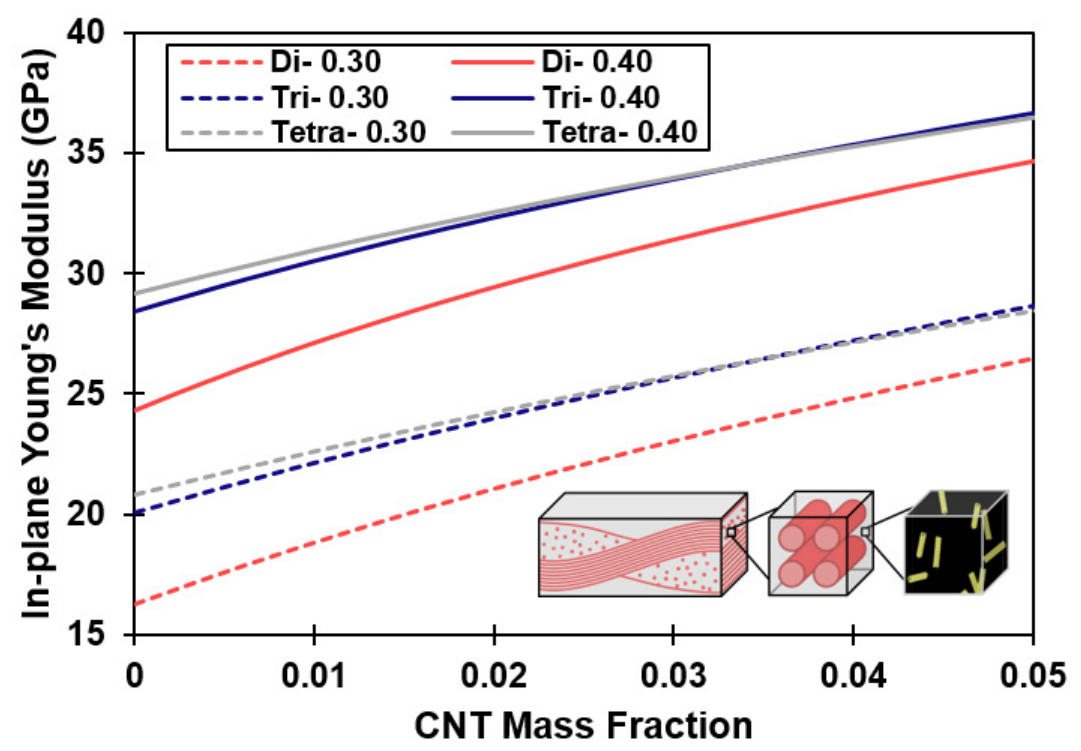

Figure 5-18. Predicted woven composite in-plane Young's modulus for various epoxy types and CNT concentrations. Results are shown for 0.30 overall fiber volume fraction (dashed) and 0.40 volume fraction (solid). 


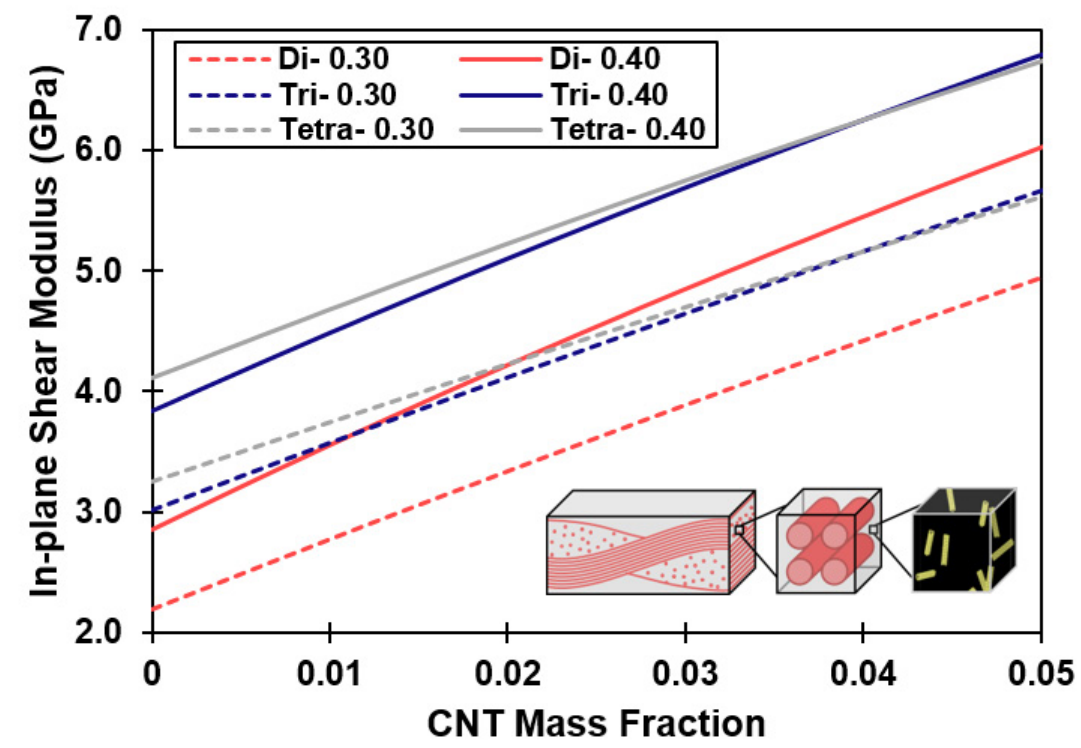

Figure 5-19. Predicted woven composite in-plane shear modulus for various epoxy types and CNT concentrations. Results are shown for 0.30 overall fiber volume fraction (dashed) and 0.40 volume fraction (solid).

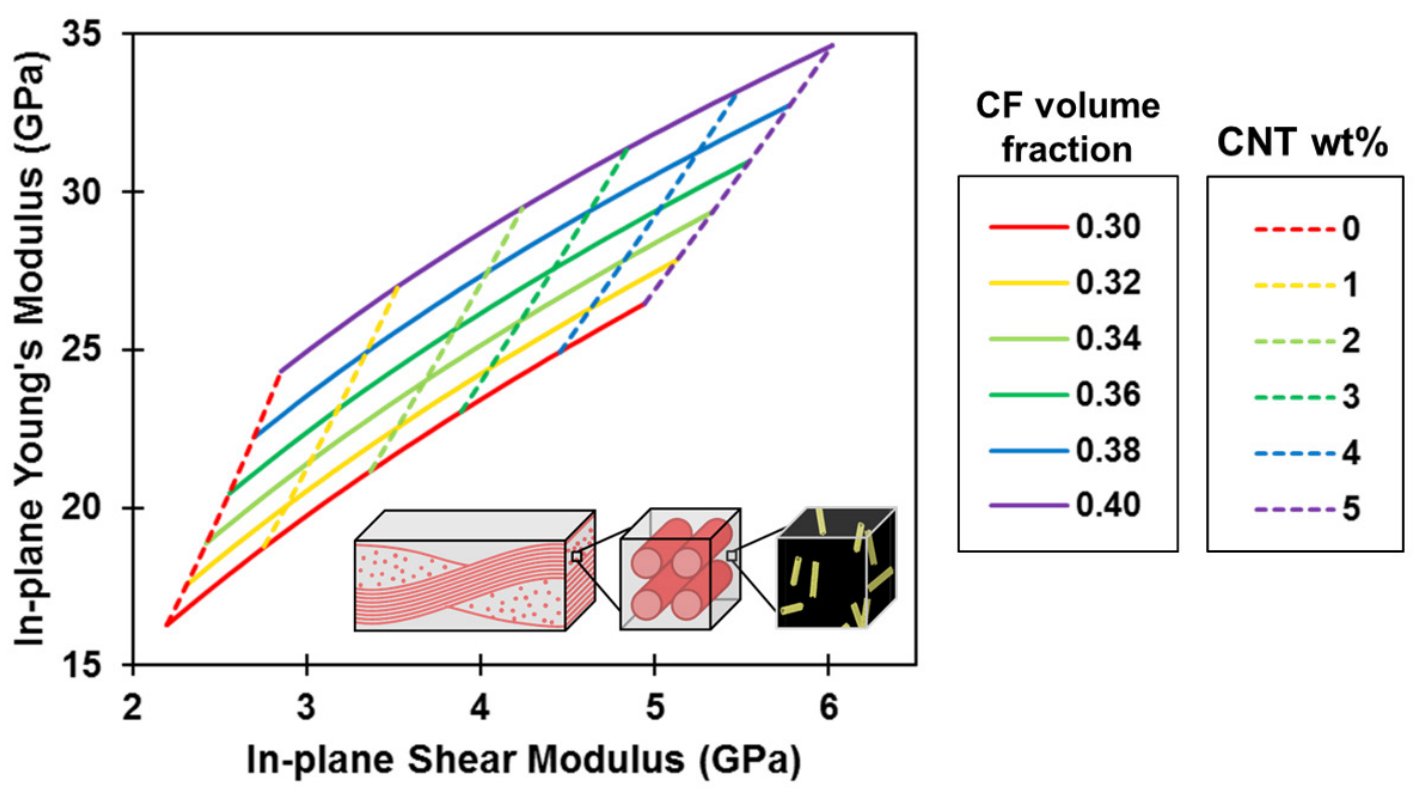

Figure 5-20. Design map for optimizing the in-plane Young's modulus and shear modulus for a woven $\mathrm{CF} / \mathrm{CNT} /$ epoxy composite with the Di epoxy. 


\subsection{Conclusions}

By simulating CNT embedded in multiple epoxy matrices, the mechanical response was compared to determine what types of epoxies may be desired for structural nanocomposites. By building MD models, the CNT-epoxy adhesion was quantified to identify its role on the mechanical response. The MD results demonstrates that the strength of the CNT-epoxy interaction correlates with the epoxy's ability to conform to the CNT and closely pack around it. Such attributes are manifested in the density of the highly dense region immediately neighboring the CNT. Analysis of the interface and interphase region revealed that the tetra-functional resin epoxy did not adhere well to CNT (as compared to the other epoxies), which was detrimental to the stiffness of the MD model. Deformation simulations demonstrate the Tri MD model paired with CNT outperforms the other models with respect to stiffness.

In order to determine whether or not the excellent properties of the CNT/Tri MD model ultimately translate into a superior nanocomposite, an atomistically informed multiscale approach was employed to predict the behavior of realistic composites. The multiscale model indicates that the Tri epoxy does indeed deliver the highest modulus nanocomposite and $\mathrm{CF} / \mathrm{CNT} /$ epoxy hybrid composites for high CNT loadings. However, for CNT concentrations up to $5 \mathrm{wt} \%$, the Tri and Tetra epoxies are expected to result in composites with comparable stiffness. For CNT composites with even higher concentrations, the Tri epoxy is more promising because of the combination of good bulk stiffness and strong interaction with CNT. Due to the relatively compliant bulk properties, the Di epoxy does not appear to have the potential to yield a nanocomposite as mechanically stiff as the other epoxies studied for CNT loadings below $17 \mathrm{wt} \%$. We assert all stated results for unfunctionalized CNT reinforcement. 


\section{Chapter 6 RATE-DEPENDENT YOUNG'S MODULUS}

\subsection{Introduction}

The limitation in feasible strain rates for MD simulations presents continuing difficulties for computational material research. Within the range of experimentally viable strain rates, the rate-dependence of mechanical properties, such as Young's modulus and yield stress, has been established. Yet it is unclear how the modeled high strain rate behavior relates to quasi-static experiments. Oftentimes the discrepancies in strain rates are simply ignored by researchers, since the predicted mechanical properties appear reasonable enough.

To understand the rate-dependence of epoxy stiffness, a linear viscoelastic constitutive model will be considered. In this chapter, an expression for the Young's Modulus as a function of strain rate will be derived using a common viscoelastic model and the implications will be discussed.

\subsection{Standard Linear Solid}

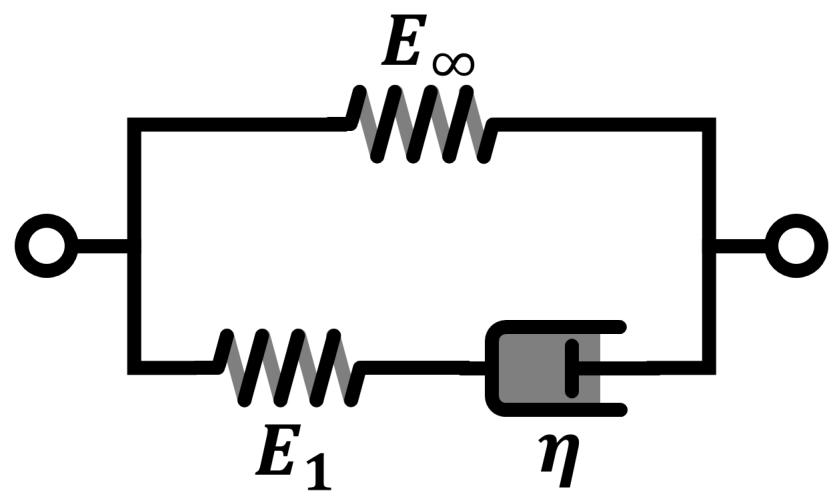

Figure 6-1. Standard Linear Solid

The Standard Linear Solid (SLS) model is shown in Figure 6-1 as an arrangement of springs and a dashpot, where $E_{\infty}$ and $E_{1}$ denote spring constants and $\eta$ is the viscosity of the dashpot. The SLS is a simple model that predicts both creep and stress relaxation. This model will be used as an example to demonstrate the resulting rate-dependent elastic 
modulus in a simplified fashion. In the next section, an expanded model will be considered that is expected to better capture typical viscoelastic behavior.

The SLS model predicts the Young's relaxation modulus $E_{R}$ as a function of time as,

$$
E_{R}(t)=E_{\infty}+E_{1} \exp \left(-\frac{t}{\tau}\right)
$$

where the relaxation time $\tau$ is given as,

$$
\tau=\frac{\eta}{E_{1}}
$$

The Boltzmann Superposition Principle states that, in the elastic regime, stress responses to successive deformations are additive. Given strain as a function of time, the resulting stress response is determined by,

$$
\sigma(t)=\int_{0}^{t} E_{R}(t-\xi) \frac{d \epsilon(\xi)}{d \xi} d \xi
$$

This principle can be utilized to estimate the Young's modulus from the Young's relaxation modulus by letting strain vary linearly with time: $\epsilon(t)=\dot{\epsilon}_{0} t$. If we take a single point on the stress-strain curve in the elastic regime at time $t_{b}$ to estimate the Young's modulus $E$, we get,

$$
E=\frac{\sigma\left(t_{b}\right)}{\epsilon\left(t_{b}\right)}=\frac{1}{t_{b}} \int_{0}^{t_{b}} E_{R}(\xi) d \xi
$$

Integrating over the Young's relaxation modulus given in Equation (6-1) yields, 


$$
E=E_{\infty}+\frac{E_{1} \tau}{t_{b}}\left[1-\exp \left(-\frac{t_{b}}{\tau}\right)\right]
$$

It can be noted that $t_{b}=\epsilon_{b} / \dot{\epsilon}_{0}$, and thus, the Young's modulus can be expressed in terms of the strain rate $\dot{\epsilon}_{0}$ as,

$$
E\left(\dot{\epsilon}_{0}\right)=E_{\infty}+\frac{E_{1} \tau}{\epsilon_{b}} \dot{\epsilon}_{0}\left[1-\exp \left(-\frac{\epsilon_{b}}{\tau \dot{\epsilon}_{0}}\right)\right]
$$

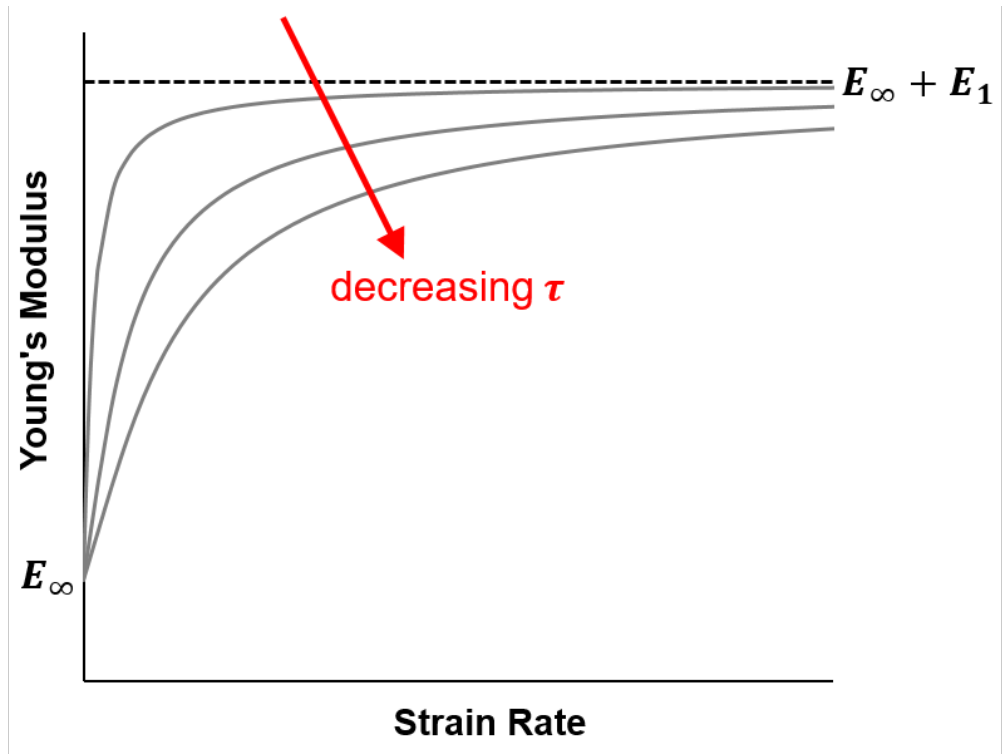

Figure 6-2. Prediction of Young's modulus vs strain rate relationship by the SLS model. Varying relaxation times shown for comparison.

The functional form of the predicted rate-dependence is shown in Figure 6-2. Different curves are shown representing varying relaxation times. As the strain rate approaches zero, the material is allowed to fully relax as it is slowly being deformed. The Young's modulus at this limit is the equilibrium modulus $E_{\infty}$. At very high strain rates, material relaxation is altogether suppressed. The effect of the dashpot diminishes, and thus, the Young's modulus approaches the sum of the spring constants, $E_{\infty}+E_{1}$. It is important to note that the SLS model predicts that the Young's modulus will converge as the strain rate increases without bound. Whether the Young's modulus will converge rapidly or not depends on the 
relaxation time. As shown in Figure 6-2, smaller relaxation times result in a more gradual convergence.

\subsection{Generalized Maxwell Model}

While the SLS model exhibits one relaxation mode, it is well known that polymers demonstrate a wide-range relaxation times. In order to account for this behavior, the SLS model can be expanded with additional springs and dashpots. Such as model is known as the Generalized Maxwell model (or Maxwell-Wiechert model) and is shown in Figure 6-3.

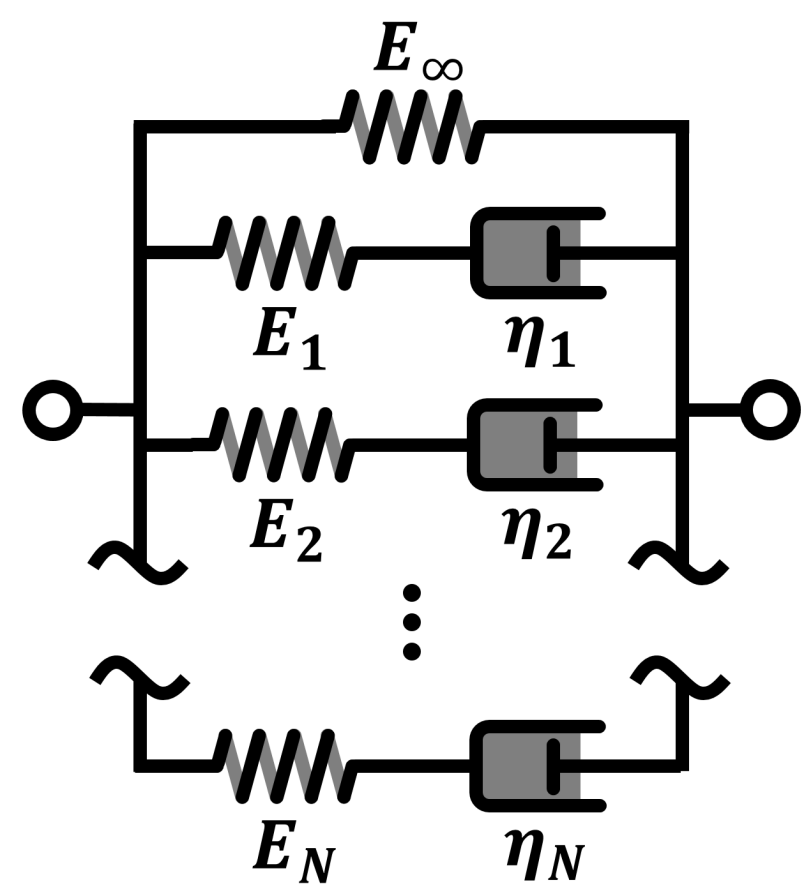

Figure 6-3. Generalized Maxwell Model

The Young's relaxation modulus of the Generalized Maxwell model is given by,

$$
E_{R}(t)=E_{\infty}+\sum_{i=1}^{N} E_{i} \exp \left(-\frac{t}{\tau_{i}}\right)
$$


where $\tau_{i}$ is given as,

$$
\tau_{i}=\frac{\eta_{i}}{E_{i}}
$$

Applying the same steps demonstrated for the SLS model, the relaxation modulus can be used to obtained an analytical expression for the Young's modulus as a function of strain rate:

$$
E\left(\dot{\epsilon}_{0}\right)=E_{\infty}+\sum_{i=1}^{N} \frac{E_{i} \tau_{i}}{\epsilon_{b}} \dot{\epsilon}_{0}\left[1-\exp \left(-\frac{\epsilon_{b}}{\tau_{i} \dot{\epsilon}_{0}}\right)\right]
$$

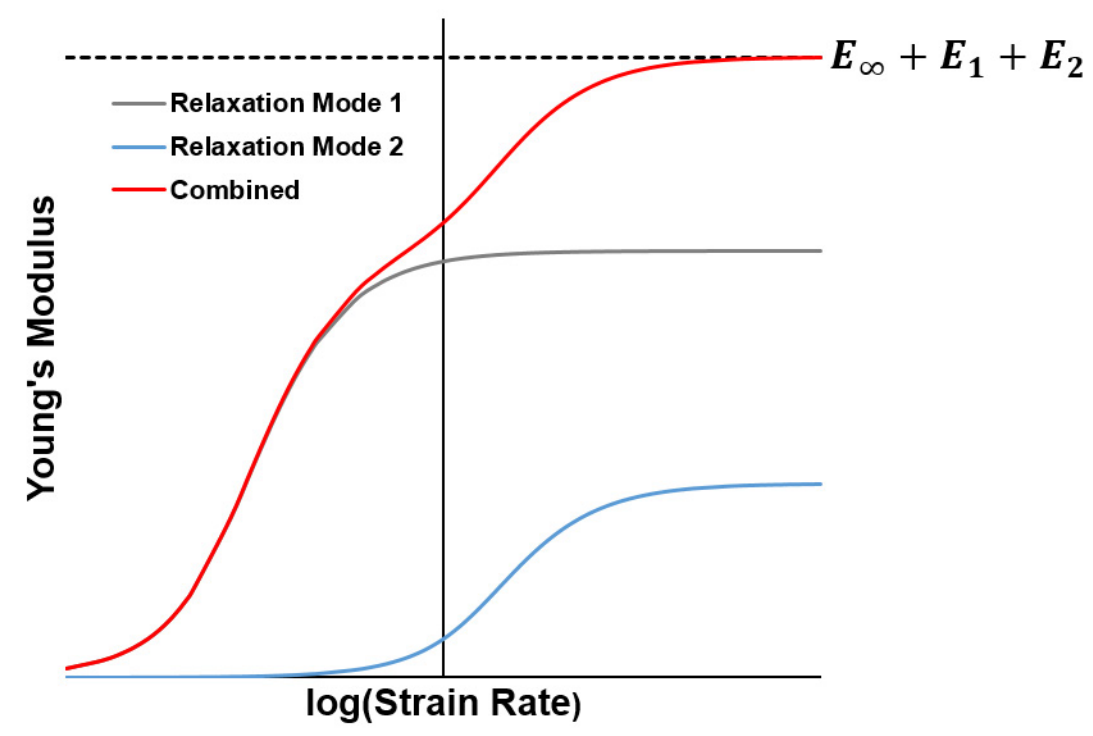

Figure 6-4. Example rate-dependence of Young's modulus given two distinct relaxation modes as predicted by the Generalized Maxwell model

To illustrate the model, Figure 6-4 provides an example of how the Young's modulus varies with strain rate given two distinct relaxation modes. Each Maxwell arm demonstrates two regimes: (1) full relaxation at low strain rates and (2) suppressed relaxation at high strain rates. The transition location between regimes depends on the relaxation time of the particular mode. Each mode provides a "smooth step" curve on the modulus versus strain 
rate plot. We can think of the mode as switching "on" and then "off". The overall response is obtained by the sum of the Maxwell arms and is shown in red in Figure 6-4. In this case, as the mode 1 curve plateaus (indicating the rate of mechanical deformation overcomes relaxation), the second mode is fast enough yet to mostly relax. However, for greater strain rates, both relaxation modes will be suppressed and the overall stiffness will converge to the sum of the spring constants, $E_{\infty}+E_{1}+E_{2}$. According to the Generalized Maxwell model, the overall curve may show "smooth steps" as consecutive modes are being switched off.

If a relaxation mode is extremely fast, it is expected to have a negligible effect on the Young's modulus for experimentally viable strain rates and would be difficult or impossible to detect. Approaching MD strain rates, it is possible that an unidentified relaxation mode "switches off" resulting in a notable increase in Young's modulus. It is desirable then to characterize as great a range of relaxation modes as can be achieved. Even so, fast relaxation modes may be missed which contribute to mechanical response at the ultra-high strain rates.

\subsection{Conclusions}

According to simple linear viscoelastic models, a broad spectrum of relaxation modes is critical to predict the evolution of the Young's modulus from experimental strain rates to simulation strain rates. Stress relaxation or creep tests are not sufficient to characterize the modes required. Perhaps, one method of finding the extensive relaxation behavior is through DMA. Baumgaertel et al. analyzed DMA frequency-sweep data of a polystyrene blend to obtain relaxation times spanning about six decades [109]. Such an analysis may be sufficient to extrapolate out to MD strain rates only if faster relaxation modes that cannot be observed in a DMA test can be assumed to have a negligible effect on the overall mechanical stiffness. 


\section{Chapter 7 RECOMMENDATIONS FOR FUTURE WORK}

\subsection{Parameterizing ReaxFF for Epoxies}

The ReaxFF parameter set used in this work was not developed to specifically predict the mechanical properties of epoxies. The Strachan et al. parameter set was developed to model energetic materials such as RDX and served as the basis of the Liu et al. parameter set used here. Liu et al. extended the parameters to correct long-range van der Waals interactions and accurately reproduce the crystal structures of various materials. Even so, the Liu et al. parameter set has been shown to provide reasonable predictions for the mechanical properties of epoxies [16]. It appears that a ReaxFF parameter set is capable of powerfully applying to a broad range of materials. However, it is expected that more accurate results would be obtained by a parameter set specially trained for epoxies. Such a parameter set would undoubtedly include the epoxy-amine reaction. Currently, crosslinked epoxies are built by externally enforcing the epoxy-amine reaction. Perhaps, a new ReaxFF set would allow the crosslinking process to be simulated without direct intervention.

\subsection{Thermal Properties of CNT/epoxy}

The CNT/epoxy simulations carried out here address the influence of CNT on the overall composite mechanical properties. As stated in Chapter 1, the low thermal conductivity of epoxy-matrix composites is another key concern to be addressed. The MD models developed can be used to study the interfacial thermal conductance between CNT and epoxy for the di-, tri-, and tetra-functional resin epoxies. The results may indicate if the thermal conductivity of a particular epoxy is especially enhanced by CNT. To obtain the interfacial thermal conductance, Varshney et al. applied a hot thermostat to a CNT and a cold thermostat to an outlying epoxy region [110]. The NVE ensemble was assigned to the region between the hot and cold thermostats. (A similar setup is described in Chapter 4 to determine the thermal conductivity of BMI.) After running an MD simulation, a steady-state temperature gradient was established which showed a drop in temperature from the CNT to 
the interphase epoxy atoms. The difference in temperature between the CNT and neighboring epoxy indicates how well phonons are transported between the nanoparticle and matrix. By using the method demonstrated by Varshney et al., the same models used for determining mechanical properties could also be used for comparing the thermal conductance of various epoxies.

\subsection{Functionalized CNT}

Since functionalization of CNT is a common way to improve CNT-matrix interactions, modeling functionalized CNT is an obvious next step. While the Tri and Tetra epoxies demonstrated superior hybrid composite moduli for various unfunctionalized CNT concentration, the same cannot necessary be said for functionalized CNT. One possible option is amine-functionalized CNT $\left(\mathrm{CNT}-\mathrm{NH}_{2}\right)$, which can covalently bond with the epoxide groups in the resin molecule. In modeling $\mathrm{CNT}-\mathrm{NH}_{2} / \mathrm{epoxy}$, the epoxy stoichiometry would likely need to be altered for optimum mechanical stiffness. If the matrix itself is a stoichiometric mixture of resin and hardener, introducing $\mathrm{CNT}-\mathrm{NH}_{2}$ would allow the nanoparticle to consume some epoxide groups and thus deprive some epoxides from reacting with hardener molecules. In this case, the crosslink density may be low, and consequently the matrix region would be compliant. For good overall stiffness, not only is good CNT-matrix interaction required, but also the bulk matrix itself must be stiff. Different resin-hardener stoichiometries may need to be attempted to determine what will provide for good bonding with $\mathrm{CNT}-\mathrm{NH}_{2}$ and maintain high crosslink density in the matrix.

\subsection{BMI Modeling}

In addition to the BMI MD model properties discussed in Chapter 4, further validation may be obtained by considering atomic vibrations. There is a wealth of IR spectroscopy data available in literature, and the dipole moment may be calculated during an MD simulation. Additionally, the atomic vibrations may be analyzed directly from the atomic velocities, but this does not distinguish whether a vibrational mode is IR active or not. 


\section{Chapter 8 REFERENCES}

1. Zhang, R., et al., Growth of half-meter long carbon nanotubes based on Schulz-Flory distribution. Acs Nano, 2013. 7(7): p. 6156-6161.

2. Sloan, J. Skinning the F-35 fighter. 2009 06/19/2017]; Available from: http://www.compositesworld.com/articles/skinning-the-f-35-fighter.

3. Mouritz, A.P., Introduction to aerospace materials. 2012: Elsevier.

4. Aglan, H., Z. Qian, and D. Mitra-Majumdar, The effect of temperature on the critical failure properties of advanced polymer composites. Polymer testing, 1992. 11(3): p. 169-184.

5. Wilson, D., PMR-15 processing, properties and problems - a review. Polymer International, 1988. 20(5): p. 405-416.

6. $\quad$ Reinhart, T.J., Engineered materials handbook. 1987: ASM international.

7. Gojny, F.H., et al., Influence of different carbon nanotubes on the mechanical properties of epoxy matrix composites-a comparative study. Composites Science and Technology, 2005. 65(15): p. 23002313.

8. Korayem, A.H., et al., Reinforcing brittle and ductile epoxy matrices using carbon nanotubes masterbatch. Composites Part a-Applied Science and Manufacturing, 2014. 61: p. 126-133.

9. $\quad \mathrm{Ci}, \mathrm{L} . J$. and J.C. Bai, The reinforcement role of carbon nanotubes in epoxy composites with different matrix stiffness. Composites Science and Technology, 2006. 66(3-4): p. 599-603.

10. de Villoria, R.G., et al., Mechanical properties of SWNT/epoxy composites using two different curing cycles. Composites Part B-Engineering, 2006. 37(4-5): p. 273-277.

11. Loos, M.R., et al., The matrix stiffness role on tensile and thermal properties of carbon nanotubes/epoxy composites. Journal of Materials Science, 2008. 43(18): p. 6064-6069.

12. Montazeri, A., et al., Viscoelastic properties of multi-walled carbon nanotube/epoxy composites using two different curing cycles. Materials \& Design, 2010. 31(7): p. 3383-3388.

13. Gude, M.R., S.G. Prolongo, and A. Urena, Effect of the epoxy/amine stoichiometry on the properties of carbon nanotube/epoxy composites. Journal of Thermal Analysis and Calorimetry, 2012. 108(2): p. 717-723.

14. Cygan, R.T., Molecular modeling in mineralogy and geochemistry. Reviews in Mineralogy and Geochemistry, 2001. 42(1): p. 1-35.

15. Van Duin, A.C., et al., ReaxFF: a reactive force field for hydrocarbons. The Journal of Physical Chemistry A, 2001. 105(41): p. 9396-9409.

16. Odegard, G.M., et al., Predicting mechanical response of crosslinked epoxy using ReaxFF. Chemical Physics Letters, 2014. 591: p. 175-178.

17. Subramanian, N., A. Rai, and A. Chattopadhyay, Atomistically informed stochastic multiscale model to predict the behavior of carbon nanotube-enhanced nanocomposites. Carbon, 2015. 94: p. 661-672.

18. Singh, S.K., et al., Thermal properties of fluorinated graphene. Physical Review B, 2013. 87(10).

19. Diao, Z., et al., ReaxFF reactive force field for molecular dynamics simulations of epoxy resin thermal decomposition with model compound. Journal of Analytical and Applied Pyrolysis, 2013. 104: p. 618624.

20. Zhang, Y.-M., et al., Research on epoxy resin decomposition under microwave heating by using ReaxFF molecular dynamics simulations. Rsc Advances, 2014. 4(33): p. 17083-17090.

21. Mattsson, T.R., et al., First-principles and classical molecular dynamics simulation of shocked polymers. Physical Review B, 2010. 81(5).

22. Rahmat, M. and P. Hubert, Carbon nanotube-polymer interactions in nanocomposites: A review. Composites Science and Technology, 2011. 72(1): p. 72-84.

23. Coto, B., et al., Influence of the geometrical properties of the carbon nanotubes on the interfacial behavior of epoxy/CNT composites: A molecular modelling approach. Computational Materials Science, 2013. 79: p. 99-104.

24. Ganesan, Y., et al., Fracture toughness of the sidewall fluorinated carbon nanotube-epoxy interface. Journal of Applied Physics, 2014. 115(22).

25. Wang, M.C., et al., Atomistic simulation of surface functionalization on the interfacial properties of graphene-polymer nanocomposites. Journal of Applied Physics, 2014. 115(12). 
26. Ahangari, M.G., et al., Electronic and mechanical properties of single-walled carbon nanotubes interacting with epoxy: A DFT study. Physica E-Low-Dimensional Systems \& Nanostructures, 2013. 48: p. 148-156.

27. Hadden, C.M., et al., Molecular modeling of EPON-862/graphite composites: Interfacial characteristics for multiple crosslink densities. Composites Science and Technology, 2013. 76: p. 9299.

28. Yang, M.J., V. Koutsos, and M. Zaiser, Interactions between polymers and carbon nanotubes: A molecular dynamics study. Journal of Physical Chemistry B, 2005. 109(20): p. 10009-10014.

29. Saha, L.C., S.A. Mian, and J. Jang, Molecular Dynamics Simulation Study on the Carbon Nanotube Interacting with a Polymer. Bulletin of the Korean Chemical Society, 2012. 33(3): p. 893-896.

30. Zaminpayma, E. and K. Mirabbaszadeh, Interaction between single-walled carbon nanotubes and polymers: A molecular dynamics simulation study with reactive force field. Computational Materials Science, 2012. 58: p. 7-11.

31. Becker, O., R. Varley, and G. Simon, Morphology, thermal relaxations and mechanical properties of layered silicate nanocomposites based upon high-functionality epoxy resins. Polymer, 2002. 43(16): p. $4365-4373$.

32. Amaral, C.R., et al., Impact of Aliphatic Amine Comonomers on DGEBA Epoxy Network Properties. Polymer Engineering and Science, 2014. 54(9): p. 2132-2138.

33. Garcia, F.G., et al., Influence of Chemical Structure of Hardener on Mechanical and Adhesive Properties of Epoxy Polymers. Journal of Applied Polymer Science, 2010. 117(4): p. 2213-2219.

34. Grishchuk, S., et al., Structure, thermal, and mechanical properties of DDM-hardened epoxy/benzoxazine hybrids: Effects of epoxy resin functionality and ETBN toughening. Journal of Applied Polymer Science, 2013. 127(6): p. 5082-5093.

35. Crawford, E. and A.J. Lesser, The effect of network architecture on the thermal and mechanical behavior of epoxy resins. Journal of Polymer Science Part B-Polymer Physics, 1998. 36(8): p. 13711382.

36. Shenogina, N.B., et al., Molecular Modeling Approach to Prediction of Thermo-Mechanical Behavior of Thermoset Polymer Networks. Macromolecules, 2012. 45(12): p. 5307-5315.

37. Nouri, N. and S. Ziaei-Rad, A Molecular Dynamics Investigation on Mechanical Properties of CrossLinked Polymer Networks. Macromolecules, 2011. 44(13): p. 5481-5489.

38. Sirk, T.W., et al., High strain rate mechanical properties of a cross-linked epoxy across the glass transition. Polymer, 2013. 54(26): p. 7048-7057.

39. Yang, S. and J. Qu, Computing thermomechanical properties of crosslinked epoxy by molecular dynamic simulations. Polymer, 2012. 53(21): p. 4806-4817.

40. Clancy, T.C., et al., Molecular modeling for calculation of mechanical properties of epoxies with moisture ingress. Polymer, 2009. 50(12): p. 2736-2742.

41. Li, C. and A. Strachan, Molecular dynamics predictions of thermal and mechanical properties of thermoset polymer EPON862/DETDA. Polymer, 2011. 52(13): p. 2920-2928.

42. Bandyopadhyay, A., et al., Molecular modeling of crosslinked epoxy polymers: The effect of crosslink density on thermomechanical properties. Polymer, 2011. 52(11): p. 2445-2452.

43. Shenogina, N.B., et al., Molecular modeling of elastic properties of thermosetting polymers using a dynamic deformation approach. Polymer, 2013. 54(13): p. 3370-3376.

44. Shokuhfar, A. and B. Arab, The effect of cross linking density on the mechanical properties and structure of the epoxy polymers: molecular dynamics simulation. Journal of Molecular Modeling, 2013. 19(9): p. 3719-3731.

45. Bandyopadhyay, A. and G.M. Odegard, Molecular modeling of crosslink distribution in epoxy polymers. Modelling and Simulation in Materials Science and Engineering, 2012. 20(4): p. 17.

46. Li, C., E. Coons, and A. Strachan, Material property prediction of thermoset polymers by molecular dynamics simulations. Acta Mechanica, 2014. 225(4-5): p. 1187-1196.

47. Tsige, M., C.D. Lorenz, and M.J. Stevens, Role of network connectivity on the mechanical properties of highly cross-linked polymers. Macromolecules, 2004. 37(22): p. 8466-8472.

48. Tsige, M. and M.J. Stevens, Effect of cross-linker functionality on the adhesion of highly cross-linked polymer networks: A molecular dynamics study of epoxies. Macromolecules, 2004. 37(2): p. 630-637. 
49. Liu, L.C., et al., ReaxFF-/g: Correction of the ReaxFF Reactive Force Field for London Dispersion, with Applications to the Equations of State for Energetic Materials. Journal of Physical Chemistry A, 2011. 115(40): p. 11016-11022.

50. Plimpton, S., Fast parallel algorithms for short-range molecular dynamics. Journal of computational physics, 1995. 117(1): p. 1-19.

51. Aktulga, H.M., et al., Parallel reactive molecular dynamics: Numerical methods and algorithmic techniques. Parallel Computing, 2012. 38(4): p. 245-259.

52. Strachan, A., et al., Shock waves in high-energy materials: The initial chemical events in nitramine $R D X$. Physical Review Letters, 2003. 91(9): p. 098301.

53. Strachan, A., et al., Thermal decomposition of $R D X$ from reactive molecular dynamics. The Journal of chemical physics, 2005. 122(5): p. 054502.

54. Jorgensen, W.L., D.S. Maxwell, and J. TiradoRives, Development and testing of the OPLS all-atom force field on conformational energetics and properties of organic liquids. Journal of the American Chemical Society, 1996. 118(45): p. 11225-11236.

55. Schneider, T. and E. Stoll, Molecular-dynamics study of a three-dimensional one-component model for distortive phase transitions. Physical Review B, 1978. 17(3): p. 1302.

56. Jensen, B.D., K.E. Wise, and G.M. Odegard, The effect of time step, thermostat, and strain rate on ReaxFF simulations of mechanical failure in diamond, graphene, and carbon nanotube. Journal of computational chemistry, 2015. 36(21): p. 1587-1596.

57. Varley, R., et al., Toughening of a trifunctional epoxy system: 1. Near infra-red spectroscopy study of homopolymer cure. Polymer, 1995. 36(7): p. 1347-1355.

58. Dannenberg, H. and W. Harp Jr, Determination of cure and analysis of cured epoxy resins. Analytical Chemistry, 1956. 28(1): p. 86-90.

59. Musto, P., et al., The curing process and moisture transport in a tetrafunctional epoxy resin as investigated by FT-NIR spectroscopy. High Performance Polymers, 2000. 12(1): p. 155-168.

60. Poisson, N., G. Lachenal, and H. Sautereau, Near-and mid-infrared spectroscopy studies of an epoxy reactive system. Vibrational spectroscopy, 1996. 12(2): p. 237-247.

61. Product Bulletin SC:1183-02. 2001, Resolution Performance Products LLC.

62. Littell, J.D., et al., Measurement of epoxy resin tension, compression, and shear stress-strain curves over a wide range of strain rates using small test specimens. Journal of Aerospace Engineering, 2008. 21(3): p. 162-173.

63. Buckley, C.P., et al., Deformation of thermosetting resins at impact rates of strain. Part I: Experimental study. Journal of the Mechanics and Physics of Solids, 2001. 49(7): p. 1517-1538.

64. Zhou, Y., et al., Experimental study on the thermal and mechanical properties of multi-walled carbon nanotube-reinforced epoxy. Materials Science and Engineering: A, 2007. 452: p. 657-664.

65. Becker, O., et al., Layered silicate nanocomposites based on various high-functionality epoxy resins: The influence of cure temperature on morphology, mechanical properties, and free volume. Macromolecules, 2003. 36(5): p. 1616-1625.

66. Lincoln, J.E., R.J. Morgan, and E.E. Shin, Moisture absorption-network structure correlations in BMPM/DABPA bismaleimide composite matrices. Journal of Advanced Materials, 2000. 32(4): p. 2434.

67. King, J.J., M. Chaudhari, and S. Zahir, A new bismaleimide system for high performance applications. Technology vectors, 1984: p. 392-408.

68. Morgan, R.J., et al., Characterization of the cure reactions of bismaleimide composite matrices. Polymer, 1997. 38(3): p. 639-646.

69. Shin, E.E., et al., Hygrothermal durability and thermal aging behavior prediction of high-temperature polymer-matrix composites and their resins. Journal of Thermoplastic Composite Materials, 2000. 13(1): p. 40-57.

70. Morgan, R.J., et al., TOUGHENING PROCEDURES, PROCESSING AND PERFORMANCE OF BISMALEIMIDE CARBON-FIBER COMPOSITES. Polymer, 1993. 34(4): p. 835-842.

71. $\mathrm{Hu}, \mathrm{X}$. and J.R. Meng, Effect of organoclay on the curing reactions in bismaleimide/diallyl bisphenol a resin. Journal of Polymer Science Part a-Polymer Chemistry, 2005. 43(5): p. 994-1006.

72. Xiong, X.H., et al., Cure kinetics and thermal properties of novel bismaleimide containing phthalide cardo structure. Thermochimica Acta, 2011. 514(1-2): p. 44-50. 
73. Mijovic, J. and S. Andjelic, Study of the mechanism and rate of bismaleimide cure by remote in-situ real time fiber optic near-infrared spectroscopy. Macromolecules, 1996. 29(1): p. 239-246.

74. Phelan, J.C. and C.S.P. Sung, Cure characterization in bis(maleimide)/diallylbisphenol A resin by fluorescence, FT-IR, and UV-reflection spectroscopy. Macromolecules, 1997. 30(22): p. 6845-6851.

75. Shibata, M., et al., High-performance bio-based bismaleimide resins using succinic acid and engenol. Polymer Journal, 2011. 43(11): p. 916-922.

76. Rozenberg, B.A., et al., The cure mechanism of the 4,4'-(N,N'-bismaleimide) diphenylmethane-2,2'diallylbisphenol A system. Polymer Science Series A, 2001. 43(4): p. 386-399.

77. Reyx, D., et al., Thermal reaction between N-phenylmaleimide and 2-allylphenol as a model for the crosslinking reaction in bismaleimide polymerization with diallylbisphenol A. Macromolecular Chemistry and Physics, 1995. 196(3): p. 775-785.

78. Rozenberg, B.A., et al., High-performance bismaleimide matrices: Cure kinetics and mechanism. Polymers for Advanced Technologies, 2002. 13(10-12): p. 837-844.

79. Shibahara, S., et al., Thermal reactions of $\mathrm{N}$-phenylmaleimide and mono- or di-functional allylphenols. Polymer Journal, 1998. 30(5): p. 404-409.

80. Zahir, S., M.A. Chaudhari, and J. King, NOVEL HIGH-TEMPERATURE RESINS BASED ON BIS(4MALEIMIDOPHENYL)METHANE. Makromolekulare Chemie-Macromolecular Symposia, 1989. 25 : p. 141-154.

81. Shibahara, S., et al., Curing reactions of bismaleimidodiphenylmethane with mono- or di-functional allylphenols. High resolution solid-state C-13 NMR study. Polymer Journal, 1998. 30(5): p. 410-413.

82. Enoki, T., T. Takeda, and K. Ishii, Effects of catalysts on the model reactions of several maleimide resin systems (III): allyl-modified maleimniide. High Performance Polymers, 1995. 7(4): p. 399-410.

83. Varshney, V., A.K. Roy, and J.W. Baur, Molecular Modeling of Thermo-Physical Properties of BMI Matrix and its Interface with Amorphous Carbon Fiber, in Proceedings of the American Society for Composites 2014-Twenty-ninth Technical Conference of Composite Materials, H. Kim, et al., Editors. 2014, DEStech Publications: San Diego, CA.

84. Varshney, V., A.K. Roy, and J.W. Baur, Modeling the Role of Bulk and Surface Characteristics of Carbon Fiber on Thermal Conductance across the Carbon-Fiber/Matrix Interface. Acs Applied Materials \& Interfaces, 2015. 7(48): p. 26674-26683.

85. Fan, H.B. and M.M.F. Yuen, Material properties of the cross-linked epoxy resin compound predicted by molecular dynamics simulation. Polymer, 2007. 48(7): p. 2174-2178.

86. Lin, P.-H. and R. Khare, Molecular simulation of cross-linked epoxy and epoxy- POSS nanocomposite. Macromolecules, 2009. 42(12): p. 4319-4327.

87. Varshney, V., et al., A molecular dynamics study of epoxy-based networks: Cross-linking procedure and prediction of molecular and material properties. Macromolecules, 2008. 41(18): p. 6837-6842.

88. Komarov, P.V., et al., Highly cross-linked epoxy resins: an atomistic molecular dynamics simulation combined with a mapping/reverse mapping procedure. Macromolecules, 2007. 40(22): p. 8104-8113.

89. Cornell, W.D., et al., A second generation force field for the simulation of proteins, nucleic acids, and organic molecules (vol 117, pg 5179, 1995). Journal of the American Chemical Society, 1996. 118(9): p. 2309-2309.

90. Wang, J.M., et al., Development and testing of a general amber force field. Journal of Computational Chemistry, 2004. 25(9): p. 1157-1174.

91. Muggeo, V.M.R., Estimating regression models with unknown break-points. Statistics in Medicine, 2003. 22(19): p. 3055-3071.

92. Gao, Y.W., et al., High-performance hexagonal boron nitride/bismaleimide composites with high thermal conductivity, low coefficient of thermal expansion, and low dielectric loss. Polymers for Advanced Technologies, 2012. 23(5): p. 919-928.

93. Wu, C.F., Competitive Absorption of Epoxy Monomers on Carbon Nanotube: A Molecular Simulation Study. Journal of Polymer Science Part B-Polymer Physics, 2011. 49(15): p. 1123-1130.

94. Liu, W., et al., Interactions between single-walled carbon nanotubes and polyethylene/polypropylene/polystyrene/poly(phenylacetylene)/poly(p-phen ylenevinylene) considering repeat unit arrangements and conformations: a molecular dynamics simulation study. Journal of Physical Chemistry C, 2008. 112(6): p. 1803-1811. 
95. Rouhi, S., Y. Alizadeh, and R. Ansari, On the Wrapping of Polyglycolide, Poly(Ethylene Oxide), and Polyketone Polymer Chains Around Single-Walled Carbon Nanotubes Using Molecular Dynamics Simulations. Brazilian Journal of Physics, 2015. 45(1): p. 10-18.

96. Gou, J., et al., Study of affinities between single-walled nanotube and epoxy resin using molecular dynamics simulation. International Journal of Nanoscience, 2006. 5(01): p. 131-144.

97. Sindu, B.S. and S. Sasmal, Evaluation of mechanical characteristics of nano modified epoxy based polymers using molecular dynamics. Computational Materials Science, 2015. 96: p. 146-158.

98. Li, K., et al., Influence of cross-linking density on the structure and properties of the interphase within supported ultrathin epoxy films. Journal of Materials Science, 2016. 51(19): p. 9019-9030.

99. Radue, M., et al. Applying Reactive Molecular Dynamics to Predict and Compare the Mechanical Response of Di-, Tri-, and Tetra-functional Resin Epoxies. in American Society of Composites-30th Technical Conference. 2015.

100. Hadden, C.M., et al., Mechanical properties of graphene nanoplatelet/carbon fiber/epoxy hybrid composites: Multiscale modeling and experiments. Carbon, 2015. 95: p. 100-112.

101. Bednarcyk, B.A. and S.M. Arnold, MAC/GMC 4. 0 User's Manual: Keywords Manual. 2002.

102. Christensen, R. and F. Waals, Effective stiffness of randomly oriented fibre composites. Journal of Composite Materials, 1972. 6(3): p. 518-535.

103. Bednarcyk, B.A. and S.M. Arnold, Micromechanics-based modeling of woven polymer matrix composites. AIAA journal, 2003. 41(9): p. 1788-1796.

104. Sun, L., et al., Mechanical properties of surface-functionalized SWCNT/epoxy composites. Carbon, 2008. 46(2): p. 320-328.

105. Wang, S., et al., Effective amino-functionalization of carbon nanotubes for reinforcing epoxy polymer composites. Nanotechnology, 2006. 17(6): p. 1551.

106. Shen, J., C. Zeng, and L.J. Lee, Synthesis of polystyrene-carbon nanofibers nanocomposite foams. Polymer, 2005. 46(14): p. 5218-5224.

107. Wang, S., et al., Covalent addition of diethyltoluenediamines onto carbon nanotubes for composite application. Polymer composites, 2009. 30(8): p. 1050-1057.

108. Bradford, P.D., et al., A novel approach to fabricate high volume fraction nanocomposites with long aligned carbon nanotubes. Composites Science and Technology, 2010. 70(13): p. 1980-1985.

109. Baumgaertel, M. and H. Winter, Determination of discrete relaxation and retardation time spectra from dynamic mechanical data. Rheologica Acta, 1989. 28(6): p. 511-519.

110. Varshney, V., et al., Effect of curing and functionalization on the interface thermal conductance in carbon nanotube-epoxy composites. Jom, 2013. 65(2): p. 140-146. 


\section{Appendix A Computational Resources}

\section{A.1 HPC Specifications}

\begin{tabular}{|l|l|l|l|}
\hline & Superior & Lightning & Thunder \\
\hline Organization & Michigan Tech & DoD, AFRL & DoD, AFRL \\
No. of standard compute nodes & 72 & 2370 & 3216 \\
Cores/node & 16 & 24 & 36 \\
Core type & Intel Xeon E5-2670 & Intel Xeon E5-2697 & Intel Xeon E5-2699 \\
& Sandy Bridge-EP & v2 Ivy Bridge-EP & v3 Haswell-EP \\
Core speed (GHz) & 2.6 & 2.7 & 2.3 \\
Memory/node (GB) & 64 & 64 & 128 \\
\hline
\end{tabular}

\section{A.2 Pure Epoxy}

Number of atoms per model: 5229-5265

\begin{tabular}{|l|l|l|l|l|l|}
\hline Simulation Description & $\begin{array}{l}\text { Simulation } \\
\text { Time (ns) }\end{array}$ & $\begin{array}{l}\text { Force } \\
\text { Field }\end{array}$ & HPC & $\begin{array}{l}\text { No. of } \\
\text { Procs }\end{array}$ & $\begin{array}{l}\text { CPU } \\
\text { Time (hr) }\end{array}$ \\
\hline Mix and densify monomers & 4.1 & OPLS & Superior & 6 & 56 \\
Crosslinking & 1.0 & OPLS & Superior & 8 & 92 \\
Import to ReaxFF and begin relaxation & 0.2 & ReaxFF & Superior & 32 & 2766 \\
Additional relaxation & 0.9 & ReaxFF & Superior & 40 & 15083 \\
Additional relaxation & 0.5 & ReaxFF & Superior & 60 & 10150 \\
Uniaxial tension & 1.0 & ReaxFF & Superior & 64 & 25135 \\
\hline
\end{tabular}

\section{A.3 BMI}

Number of atoms per model: 21,000

\begin{tabular}{|l|l|l|l|l|l|}
\hline Simulation Description & $\begin{array}{l}\text { Simulation } \\
\text { Time (ns) }\end{array}$ & $\begin{array}{l}\text { Force } \\
\text { Field }\end{array}$ & HPC & $\begin{array}{l}\text { No. of } \\
\text { Procs }\end{array}$ & $\begin{array}{l}\text { CPU Time } \\
\text { (hr) }\end{array}$ \\
\hline Mix and densify monomers & 4.1 & GAFF & Superior & 16 & 300 \\
Crosslinking part 1: BMPM reactions & 20.0 & GAFF & Superior & 16 & $1564-22071^{*}$ \\
Crosslinking part 2: Dehydration & 20.0 & GAFF & Superior & 16 & $1555-11107^{*}$ \\
Annealing & 25.1 & GAFF & Lightning & 72 & 3553 \\
Relaxation & 5.0 & GAFF & Lightning & 24 & 552 \\
Uniaxial tension & 5.0 & GAFF & Lightning & 24 & 1091 \\
\hline
\end{tabular}

*Varied widely due to different curing mechanisms implemented 


\section{A.4 CNT/epoxy}

Number of atoms per model: 5629-5665

\begin{tabular}{|l|l|l|l|l|l|}
\hline Simulation Description & $\begin{array}{l}\text { Simulation } \\
\text { Time (ns) }\end{array}$ & $\begin{array}{l}\text { Force } \\
\text { Field }\end{array}$ & HPC & $\begin{array}{l}\text { No. of } \\
\text { Procs }\end{array}$ & $\begin{array}{l}\text { CPU } \\
\text { Time (hr) }\end{array}$ \\
\hline Mix and densify monomers & 4.1 & OPLS & Superior & 16 & 83 \\
Create void for CNT & 0.1 & OPLS & Superior & 8 & 1 \\
Relax CNT/uncured epoxy & 6.0 & OPLS & Superior & 16 & 100 \\
Crosslinking & 1.0 & OPLS & Superior & 16 & 226 \\
Import to ReaxFF and begin relaxation & 0.2 & ReaxFF & Lightning & 72 & 2544 \\
Additional relaxation & 0.75 & ReaxFF & Lightning & 72 & 9811 \\
Additional relaxation & 0.75 & ReaxFF & Lightning & 72 & 9883 \\
Additional relaxation & 0.5 & ReaxFF & Lightning & 72 & 6632 \\
Interfacial analysis & 0.1 & ReaxFF & Lightning & 72 & 1003 \\
Uniaxial tension & 0.5 & ReaxFF & Lightning & 72 & 7140 \\
Simple shear & 0.25 & ReaxFF & Thunder & 48 & 3916 \\
\hline
\end{tabular}




\section{Appendix B Sample LAMMPS Scripts}

\section{B.1 Mix and Densify Monomers}

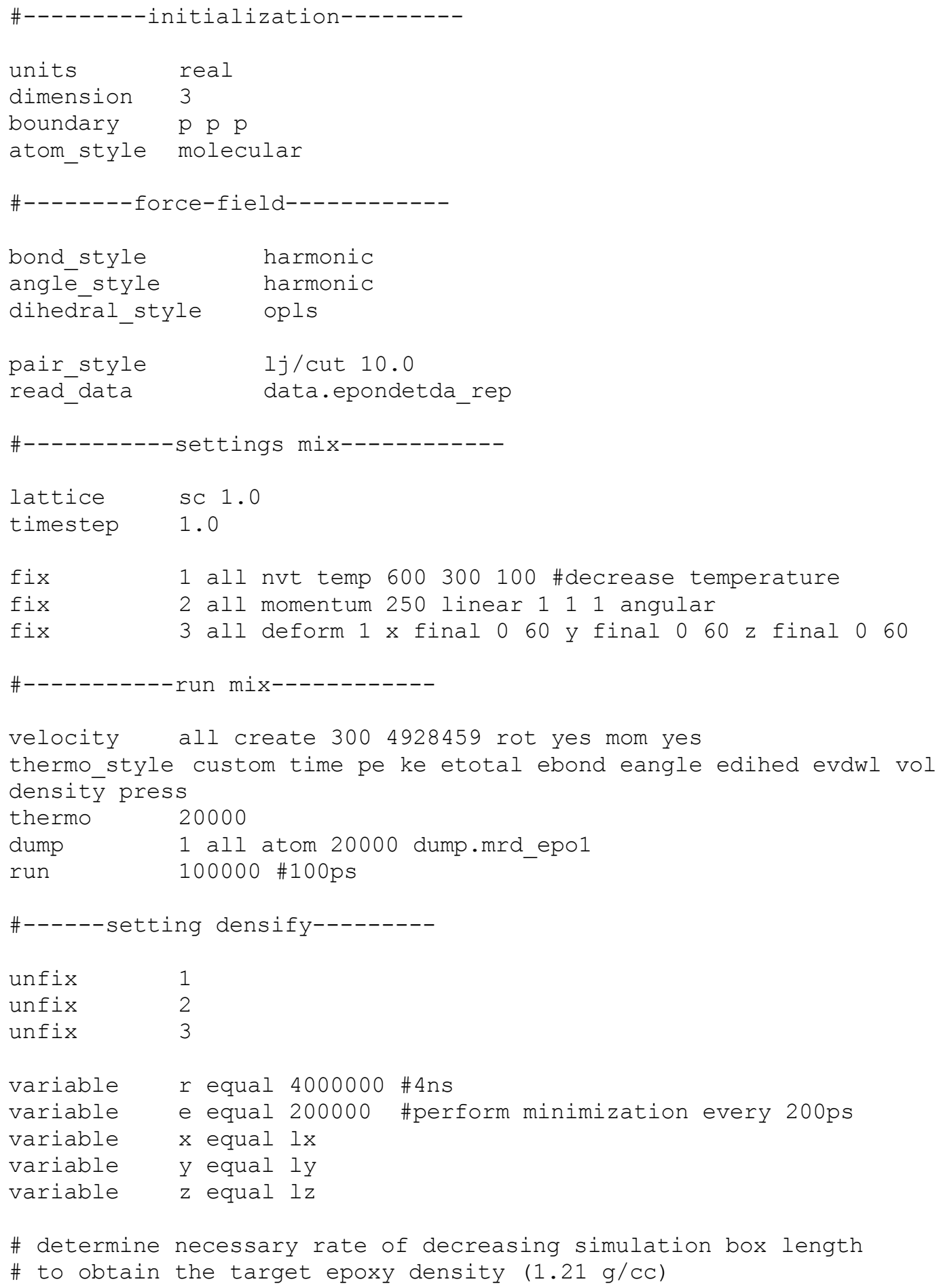




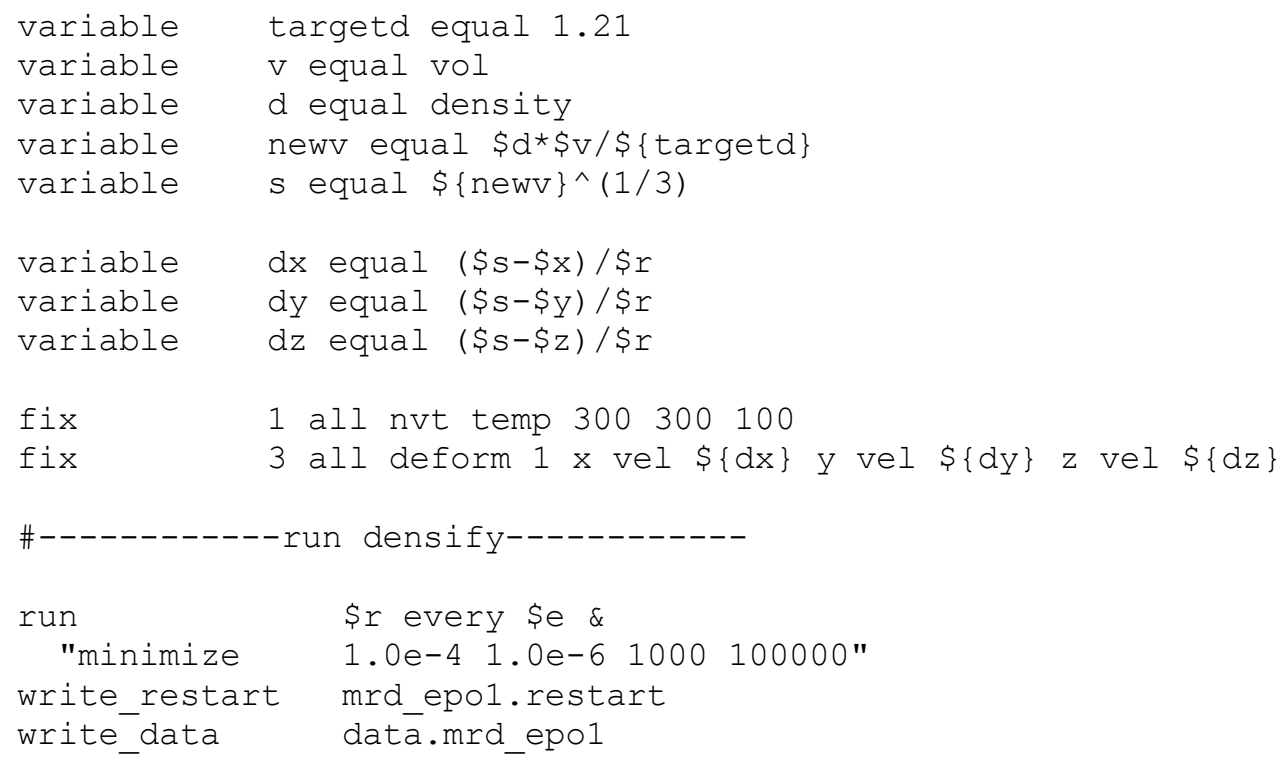

\section{B.2 Uniaxial Deformation}

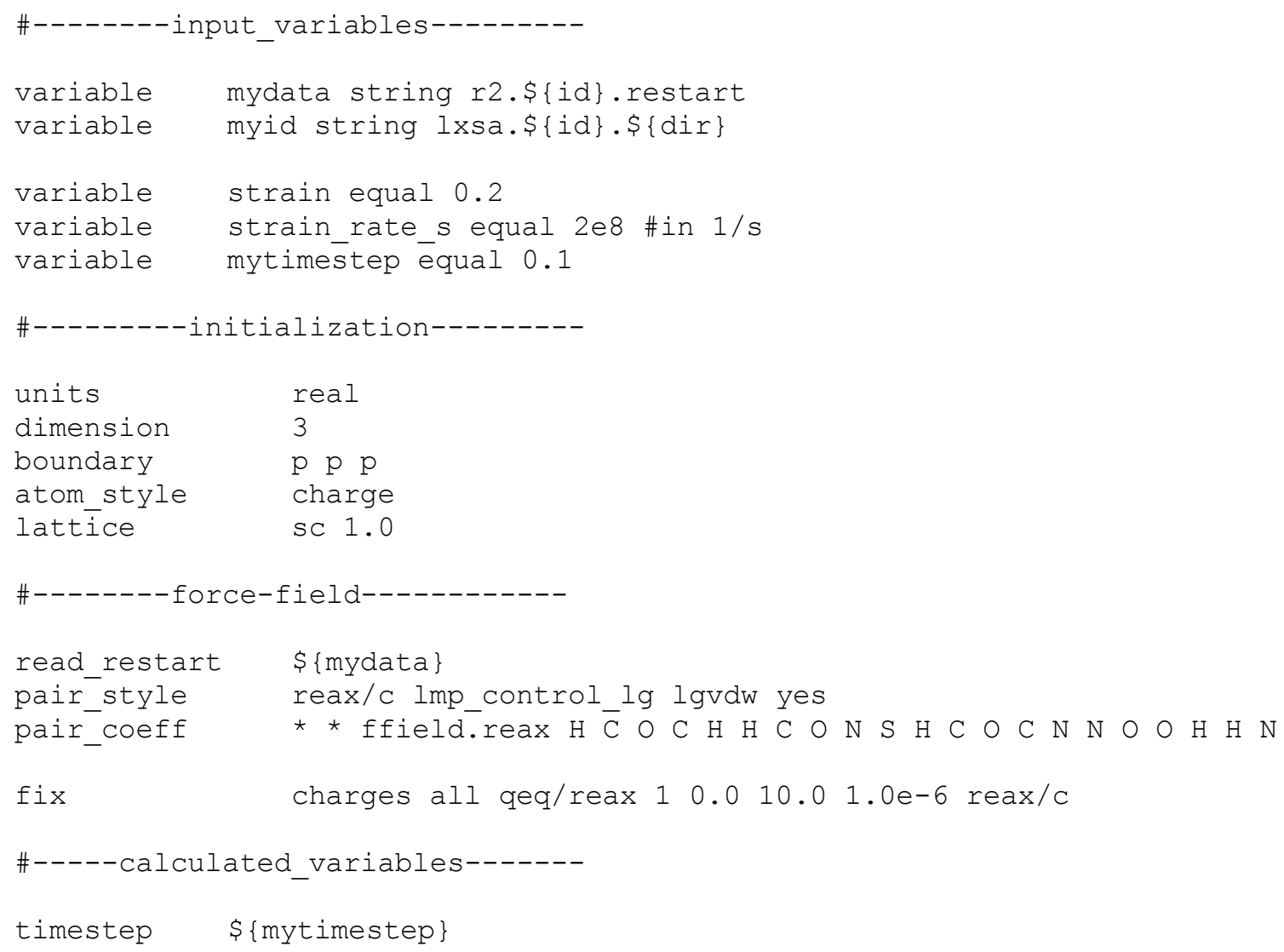




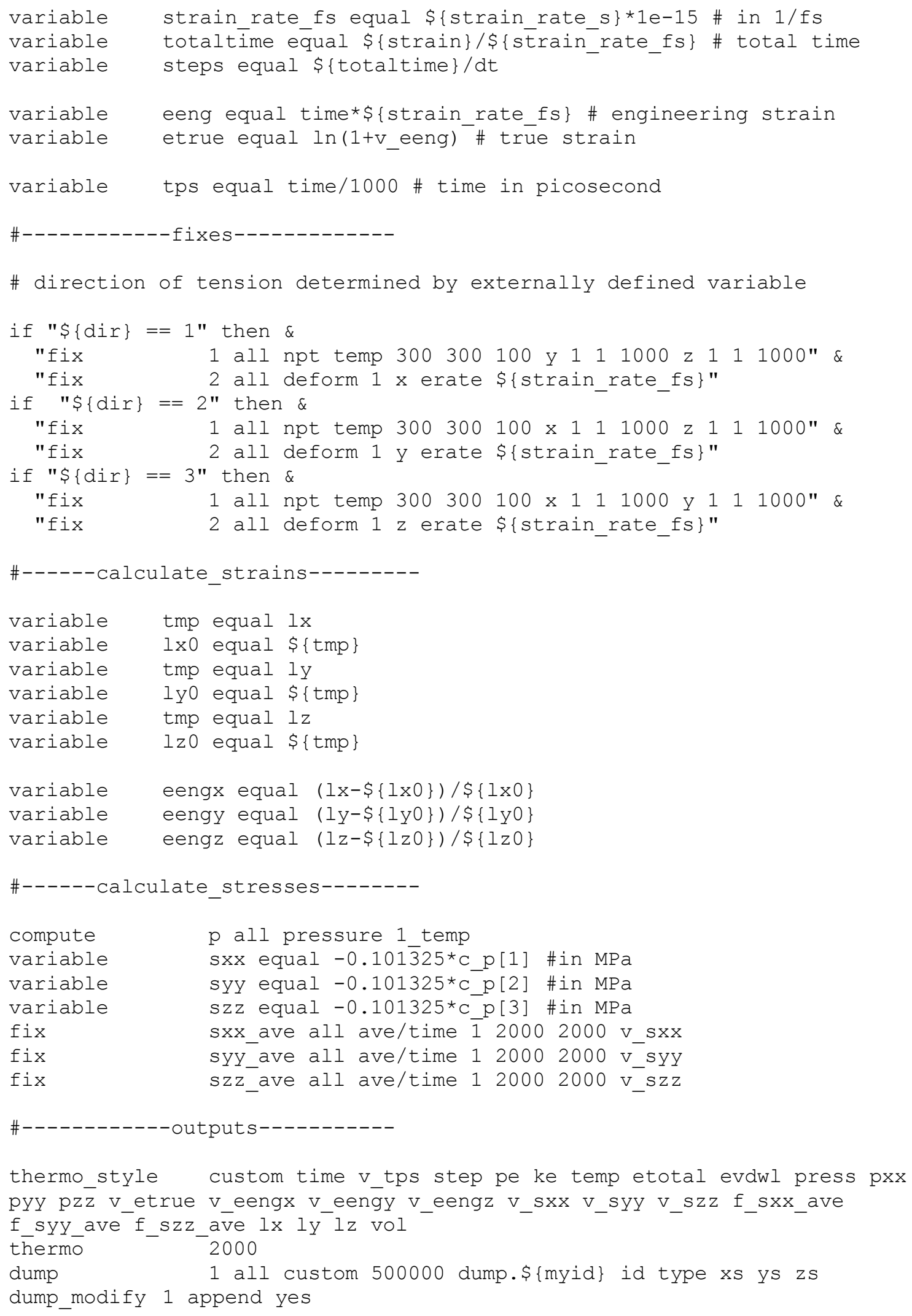


\#------------run--------------

timestep \$ \$mytimestep

run $\$\{$ steps $\}$

\#---------wrap up-------------

write_restart $\$\{$ myid .restart

write_data data.\$ $\{$ myid $\}$ 


\section{Appendix C Copyright Agreements}

\section{C.1 Copyright Agreement for Figure 2-1}

This is a License Agreement between Michigan Technological University -- Matthew Radue ("You") and Elsevier Science and Technology Journals ("Elsevier Science and Technology Journals") provided by Copyright Clearance Center ("CCC"). The license consists of your order details, the terms and conditions provided by Elsevier Science and Technology Journals, and the payment terms and conditions.

\author{
All payments must be made in full to CCC. For payment instructions, please see \\ information listed at the bottom of this form. \\ License Number \\ License date \\ Licensed content publisher \\ Licensed content title \\ Licensed content date \\ Type of Use \\ Requestor type \\ Format \\ Portion \\ Number of images/photos \\ requested \\ Title or numeric reference of the \\ portion(s) \\ 4132690046904 \\ Jun 19, 2017 \\ Elsevier Science and Technology Journals \\ Composites science and technology \\ Jan 1,1985 \\ Thesis/Dissertation \\ Academic institution \\ Print, Electronic \\ image/photo \\ 1 \\ Figure 3. The evolution of the reinforcement of carbon \\ nanotubes in epoxy composites with different matrix \\ stiffness. \\ Title of the article or chapter the The reinforcement role of carbon nanotube in epoxy \\ portion is from \\ composites with different matrix stiffness \\ Editor of portion(s) \\ $N / A$ \\ Author of portion(s) \\ Lijie $\mathrm{Ci}$, JinBo Bai \\ Volume of serial or monograph. \\ Issue, if republishing an article \\ from a serial \\ Page range of the portion \\ $599-603$ \\ Publication date of portion \\ 2006 \\ Rights for \\ Main product \\ Duration of use \\ 66 \\ 3-4 \\ Life of current edition
}


Creation of copies for the no

disabled

With minor editing privileges no

For distribution to

Worldwide

In the following language(s) Original language of publication

With incidental promotional use no

The lifetime unit quantity of new Up to 499

product

Made available in the following Research

markets

The requesting

person/organization is:

Michigan Technological University

Order reference number

Author/Editor

Matthew S Radue

The standard identifier of New

not known

Work

Title of New Work

Molecular Modeling of Aerospace Polymer Matrices Including Carbon Nanotube-Enhanced Epoxy

Publisher of New Work

Michigan Technological University

Expected publication date

Aug 2017

Estimated size (pages)

120

Total (may include CCC user fee) 0.00 USD

Terms and Conditions 


\section{TERMS AND CONDITIONS \\ The following terms are individual to this publisher:}

None

\section{Other Terms and Conditions: STANDARD TERMS AND CONDITIONS}

1. Description of Service; Defined Terms. This Republication License enables the User to obtain licenses for republication of one or more copyrighted works as described in detail on the relevant Order Confirmation (the "Work(s)"). Copyright Clearance Center, Inc. ("CCC") grants licenses through the Service on behalf of the rightsholder identified on the Order Confirmation (the "Rightsholder"). "Republication", as used herein, generally means the inclusion of a Work, in whole or in part, in a new work or works, also as described on the Order Confirmation. "User", as used herein, means the person or entity making such republication.

2. The terms set forth in the relevant Order Confirmation, and any terms set by the Rightsholder with respect to a particular Work, govern the terms of use of Works in connection with the Service. By using the Service, the person transacting for a republication license on behalf of the User represents and warrants that he/she/it (a) has been duly authorized by the User to accept, and hereby does accept, all such terms and conditions on behalf of User, and (b) shall inform User of all such terms and conditions. In the event such person is a "freelancer" or other third party independent of User and CCC, such party shall be deemed jointly a "User" for purposes of these terms and conditions. In any event, User shall be deemed to have accepted and agreed to all such terms and conditions if User republishes the Work in any fashion.

\section{Scope of License; Limitations and Obligations.}

3.1 All Works and all rights therein, including copyright rights, remain the sole and exclusive property of the Rightsholder. The license created by the exchange of an Order Confirmation (and/or any invoice) and payment by User of the full amount set forth on that document includes only those rights expressly set forth in the Order Confirmation and in these terms and conditions, and conveys no other rights in the Work(s) to User. All rights not expressly granted are hereby reserved.

3.2 General Payment Terms: You may pay by credit card or through an account with us payable at the end of the month. If you and we agree that you may establish a standing account with CCC, then the following terms apply: Remit Payment to: Copyright Clearance Center, 29118 Network Place, Chicago, IL 60673-1291. Payments Due: Invoices are payable upon their delivery to you (or upon our notice to you that they are available to you for downloading). After 30 days, outstanding amounts will be subject to a service charge of $1-1 / 2 \%$ per month or, if less, the maximum rate allowed by applicable law. Unless otherwise specifically set forth in the Order Confirmation or in a separate written agreement signed by CCC, invoices are due and payable on "net 30" terms. While User may exercise the rights licensed immediately upon issuance of the Order Confirmation, the license is automatically revoked and is null and void, as if it had never been issued, if complete payment for the license is not received on a timely basis either from User directly or through a payment agent, such as a credit card company. 3.3 Unless otherwise provided in the Order Confirmation, any grant of rights to User (i) is "one-time" (including the editions and product family specified in the license), (ii) is 
non-exclusive and non-transferable and (iii) is subject to any and all limitations and restrictions (such as, but not limited to, limitations on duration of use or circulation) included in the Order Confirmation or invoice and/or in these terms and conditions. Upon completion of the licensed use, User shall either secure a new permission for further use of the Work(s) or immediately cease any new use of the Work(s) and shall render inaccessible (such as by deleting or by removing or severing links or other locators) any further copies of the Work (except for copies printed on paper in accordance with this license and still in User's stock at the end of such period). 3.4 In the event that the material for which a republication license is sought includes third party materials (such as photographs, illustrations, graphs, inserts and similar materials) which are identified in such material as having been used by permission, User is responsible for identifying, and seeking separate licenses (under this Service or otherwise) for, any of such third party materials; without a separate license, such third party materials may not be used.

3.5 Use of proper copyright notice for a Work is required as a condition of any license granted under the Service. Unless otherwise provided in the Order Confirmation, a proper copyright notice will read substantially as follows: "Republished with permission of [Rightsholder's name], from [Work's title, author, volume, edition number and year of copyright]; permission conveyed through Copyright Clearance Center, Inc. " Such notice must be provided in a reasonably legible font size and must be placed either immediately adjacent to the Work as used (for example, as part of a by-line or footnote but not as a separate electronic link) or in the place where substantially all other credits or notices for the new work containing the republished Work are located. Failure to include the required notice results in loss to the Rightsholder and CCC, and the User shall be liable to pay liquidated damages for each such failure equal to twice the use fee specified in the Order Confirmation, in addition to the use fee itself and any other fees and charges specified.

3.6 User may only make alterations to the Work if and as expressly set forth in the Order Confirmation. No Work may be used in any way that is defamatory, violates the rights of third parties (including such third parties' rights of copyright, privacy, publicity, or other tangible or intangible property), or is otherwise illegal, sexually explicit or obscene. In addition, User may not conjoin a Work with any other material that may result in damage to the reputation of the Rightsholder. User agrees to inform CCC if it becomes aware of any infringement of any rights in a Work and to cooperate with any reasonable request of CCC or the Rightsholder in connection therewith.

4. Indemnity. User hereby indemnifies and agrees to defend the Rightsholder and CCC, and their respective employees and directors, against all claims, liability, damages, costs and expenses, including legal fees and expenses, arising out of any use of a Work beyond the scope of the rights granted herein, or any use of a Work which has been altered in any unauthorized way by User, including claims of defamation or infringement of rights of copyright, publicity, privacy or other tangible or intangible property.

5. Limitation of Liability. UNDER NO CIRCUMSTANCES WILL CCC OR THE RIGHTSHOLDER BE LIABLE FOR ANY DIRECT, INDIRECT, CONSEQUENTIAL OR INCIDENTAL DAMAGES (INCLUDING WITHOUT LIMITATION DAMAGES FOR LOSS OF BUSINESS PROFITS OR INFORMATION, OR FOR BUSINESS 
INTERRUPTION) ARISING OUT OF THE USE OR INABILITY TO USE A WORK, EVEN IF ONE OF THEM HAS BEEN ADVISED OF THE POSSIBILITY OF SUCH DAMAGES. In any event, the total liability of the Rightsholder and CCC (including their respective employees and directors) shall not exceed the total amount actually paid by User for this license. User assumes full liability for the actions and omissions of its principals, employees, agents, affiliates, successors and assigns.

6. Limited Warranties. THE WORK(S) AND RIGHT(S) ARE PROVIDED “AS IS". CCC HAS THE RIGHT TO GRANT TO USER THE RIGHTS GRANTED IN THE ORDER CONFIRMATION DOCUMENT. CCC AND THE RIGHTSHOLDER DISCLAIM ALL OTHER WARRANTIES RELATING TO THE WORK(S) AND RIGHT(S), EITHER EXPRESS OR IMPLIED, INCLUDING WITHOUT LIMITATION IMPLIED WARRANTIES OF MERCHANTABILITY OR FITNESS FOR A PARTICULAR PURPOSE. ADDITIONAL RIGHTS MAY BE REQUIRED TO USE ILLUSTRATIONS, GRAPHS, PHOTOGRAPHS, ABSTRACTS, INSERTS OR OTHER PORTIONS OF THE WORK (AS OPPOSED TO THE ENTIRE WORK) IN A MANNER CONTEMPLATED BY USER; USER UNDERSTANDS AND AGREES THAT NEITHER CCC NOR THE RIGHTSHOLDER MAY HAVE SUCH ADDITIONAL RIGHTS TO GRANT.

7. Effect of Breach. Any failure by User to pay any amount when due, or any use by User of a Work beyond the scope of the license set forth in the Order Confirmation and/or these terms and conditions, shall be a material breach of the license created by the Order Confirmation and these terms and conditions. Any breach not cured within 30 days of written notice thereof shall result in immediate termination of such license without further notice. Any unauthorized (but licensable) use of a Work that is terminated immediately upon notice thereof may be liquidated by payment of the Rightsholder's ordinary license price therefor; any unauthorized (and unlicensable) use that is not terminated immediately for any reason (including, for example, because materials containing the Work cannot reasonably be recalled) will be subject to all remedies available at law or in equity, but in no event to a payment of less than three times the Rightsholder's ordinary license price for the most closely analogous licensable use plus Rightsholder's and/or CCC's costs and expenses incurred in collecting such payment.

\section{Miscellaneous.}

8.1 User acknowledges that $\mathrm{CCC}$ may, from time to time, make changes or additions to the Service or to these terms and conditions, and CCC reserves the right to send notice to the User by electronic mail or otherwise for the purposes of notifying User of such changes or additions; provided that any such changes or additions shall not apply to permissions already secured and paid for.

8.2 Use of User-related information collected through the Service is governed by CCC's privacy policy, available online here:http://www.copyright.com/content/cc3/en/tools/footer/privacypolicy.html. 8.3 The licensing transaction described in the Order Confirmation is personal to User. Therefore, User may not assign or transfer to any other person (whether a natural person or an organization of any kind) the license created by the Order Confirmation and these terms and conditions or any rights granted hereunder; provided, however, that User may 
assign such license in its entirety on written notice to CCC in the event of a transfer of all or substantially all of User's rights in the new material which includes the Work(s) licensed under this Service.

8.4 No amendment or waiver of any terms is binding unless set forth in writing and signed by the parties. The Rightsholder and CCC hereby object to any terms contained in any writing prepared by the User or its principals, employees, agents or affiliates and purporting to govern or otherwise relate to the licensing transaction described in the Order Confirmation, which terms are in any way inconsistent with any terms set forth in the Order Confirmation and/or in these terms and conditions or CCC's standard operating procedures, whether such writing is prepared prior to, simultaneously with or subsequent to the Order Confirmation, and whether such writing appears on a copy of the Order Confirmation or in a separate instrument.

8.5 The licensing transaction described in the Order Confirmation document shall be governed by and construed under the law of the State of New York, USA, without regard to the principles thereof of conflicts of law. Any case, controversy, suit, action, or proceeding arising out of, in connection with, or related to such licensing transaction shall be brought, at CCC's sole discretion, in any federal or state court located in the County of New York, State of New York, USA, or in any federal or state court whose geographical jurisdiction covers the location of the Rightsholder set forth in the Order Confirmation. The parties expressly submit to the personal jurisdiction and venue of each such federal or state court.If you have any comments or questions about the Service or Copyright Clearance Center, please contact us at $978-750-8400$ or send an e-mail to info@copyright.com.

$\mathrm{v} 1.1$

Questions? customercare@copyright.com or +1-855-239-3415 (toll free in the US) or +1-978-646-2777. 


\section{C.2 Copyright Agreement for Figure 2-2}

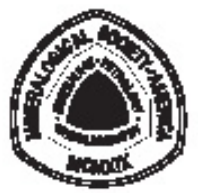

\section{MINERALOGICAL SOCIETY OF AMERICA}

3635 Concorde Pkwy Ste 500 - Chantilly VA 20151-1110 - USA Tel: 1 (703) 652-9950 - Fax: 1 (703) 652-9951 - Internet: www.minsocam.org

Matthew Scott Radue

June 20, 2017

Michigan Technological University

1400 Townsend Drive

Houghton MI 49931

United States

Email: msradue@mtu.edu

Dear Mr. Radue:

I received your e-mail message of 2017-06-20 requesting permission to reproduce the following figure in your your thesis, "Molecular Modeling of Aerospace Polymer Matrices Including Carbon Nanotube-Enhanced Epoxy" for the Michigan Technological University:

- Figure Number(s): 3 from Randall T. Cygan (2001) Molecular Modeling in Mineralogy and Geochemistry, Reviews in Mineralogy and Geochemistry, v. 42 , i. 1 , p. $1-35$.

It is with pleasure that we grant you permission to reproduce this figure without cost and all subsequent editions of the work, its ancillaries, and other derivative works, in any form or medium, whether now known or hereafter developed, in all languages, for distribution throughout the world on the conditions that reference is given to the original publication of the Mineralogical Society of America.

Sincerely,

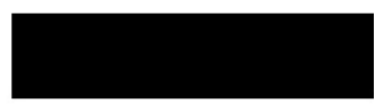

J. Alexander Speer

Executive Director, MSA 


\section{C.3 Copyright Agreement for Figure 5-12}

This is a License Agreement between Michigan Technological University -- Matthew Radue ("You") and American Inst of Aeronautics and Astronautics (AIAA) ("American Inst of Aeronautics and Astronautics (AIAA)") provided by Copyright Clearance Center ("CCC"). The license consists of your order details, the terms and conditions provided by American Inst of Aeronautics and Astronautics (AIAA), and the payment terms and conditions.

\section{All payments must be made in full to CCC. For payment instructions, please see information listed at the bottom of this form.}

License Number

License date

Licensed content publisher

Licensed content title

Licensed content date

Type of Use

Requestor type

Format

Portion

Number of images/photos requested

Title or numeric reference of the portion(s)

Title of the article or chapter the portion is from

Editor of portion(s)

Author of portion(s)

Volume of serial or monograph. 41

Issue, if republishing an article 9

from a serial

Page range of the portion

Publication date of portion

Rights for

Duration of use

Creation of copies for the disabled

With minor editing privileges

For distribution to

1

no

no
4130820396080

Jun 16, 2017

American Inst of Aeronautics and Astronautics (AIAA)

AIAA journal

Jan 1, 1963

Thesis/Dissertation

Academic institution

Print, Electronic

image/photo

Figure 3. MAC/GMC repeating unit cell used to represent a plain-weave composite

Micromechanics-Based Modeling of Woven Polymer Matrix Composites

N/A

Brett A. Bednarcyk, Steven M. Arnold

1791

Septemeber 2003

Main product

Life of current edition

Worldwide 


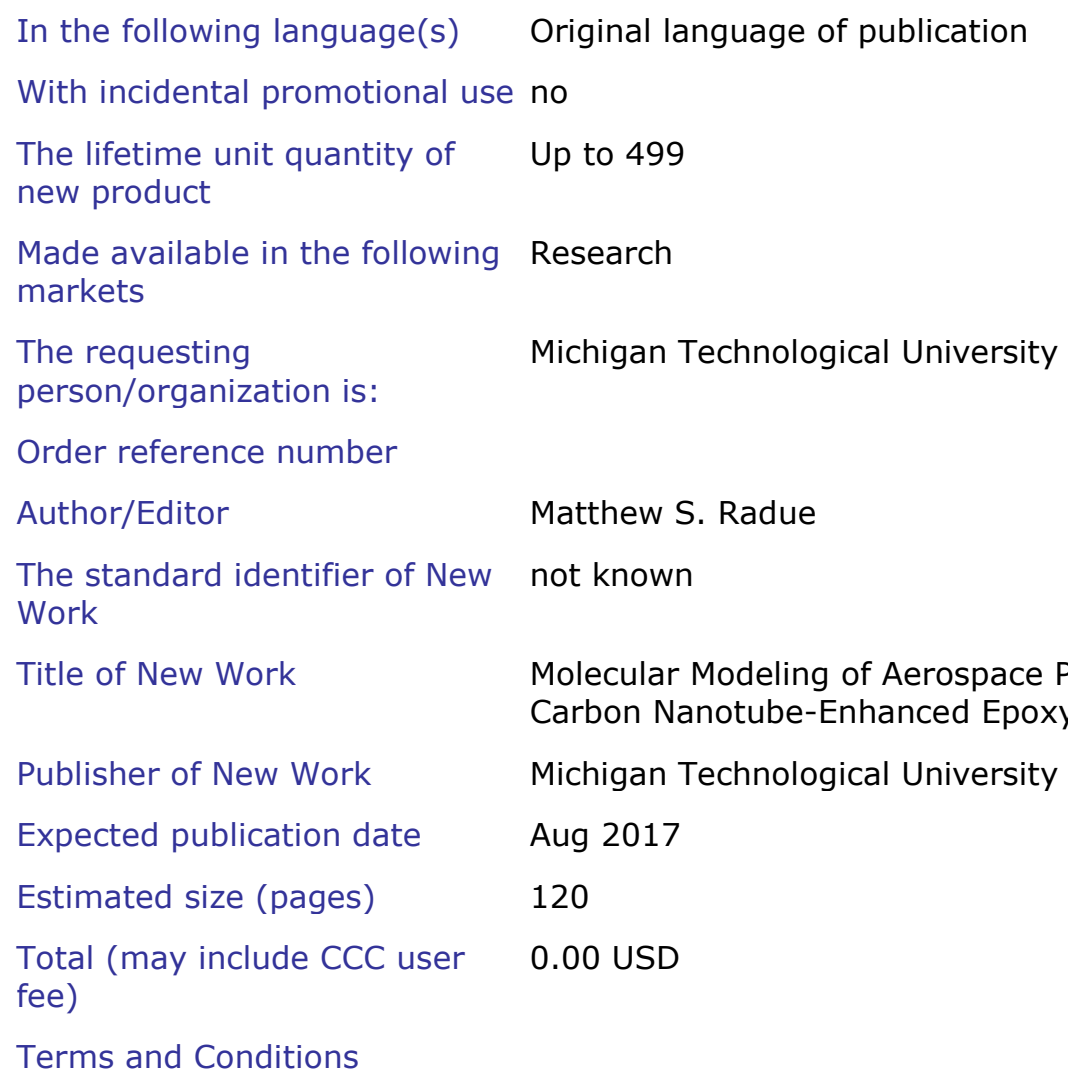

\section{TERMS AND CONDITIONS}

The following terms are individual to this publisher:

Verification of copyright ownership is your responsibility. You should only submit requests for materials that are owned by AIAA. Please review the copyright statement for the source material before submitting a reprint permission request, to ensure that AIAA is the copyright owner:

- For AIAA meeting papers, journal papers, or books with independently authored chapters (e.g., many Progress Series volumes), look at the bottom of the first fulltext page (not the cover page). There will be a footnote indicating who holds copyright.

- For other books, look at the copyright statement on the back of the title page.

If the statement reads "Copyright by the American Institute of Aeronautics and Astronautics, Inc.," then AIAA is the copyright owner, and you may submit your request.

If the statement reads otherwise, AIAA does not hold copyright, and cannot grant permission to reprint. You must seek permission from the copyright owner rather than AIAA.

Preferred credit line for reprinted material: From [original title and authors]; reprinted by permission of the American Institute of Aeronautics and Astronautics, Inc. Note that the original source also should be cited in full in the reference list. 


\section{Other Terms and Conditions: STANDARD TERMS AND CONDITIONS}

1. Description of Service; Defined Terms. This Republication License enables the User to obtain licenses for republication of one or more copyrighted works as described in detail on the relevant Order Confirmation (the "Work(s)"). Copyright Clearance Center, Inc. ("CCC") grants licenses through the Service on behalf of the rightsholder identified on the Order Confirmation (the "Rightsholder"). "Republication", as used herein, generally means the inclusion of a Work, in whole or in part, in a new work or works, also as described on the Order Confirmation. "User", as used herein, means the person or entity making such republication.

2. The terms set forth in the relevant Order Confirmation, and any terms set by the Rightsholder with respect to a particular Work, govern the terms of use of Works in connection with the Service. By using the Service, the person transacting for a republication license on behalf of the User represents and warrants that he/she/it (a) has been duly authorized by the User to accept, and hereby does accept, all such terms and conditions on behalf of User, and (b) shall inform User of all such terms and conditions. In the event such person is a "freelancer" or other third party independent of User and CCC, such party shall be deemed jointly a "User" for purposes of these terms and conditions. In any event, User shall be deemed to have accepted and agreed to all such terms and conditions if User republishes the Work in any fashion.

\section{Scope of License; Limitations and Obligations.}

3.1 All Works and all rights therein, including copyright rights, remain the sole and exclusive property of the Rightsholder. The license created by the exchange of an Order Confirmation (and/or any invoice) and payment by User of the full amount set forth on that document includes only those rights expressly set forth in the Order Confirmation and in these terms and conditions, and conveys no other rights in the Work(s) to User. All rights not expressly granted are hereby reserved.

3.2 General Payment Terms: You may pay by credit card or through an account with us payable at the end of the month. If you and we agree that you may establish a standing account with CCC, then the following terms apply: Remit Payment to: Copyright Clearance Center, 29118 Network Place, Chicago, IL 60673-1291. Payments Due: Invoices are payable upon their delivery to you (or upon our notice to you that they are available to you for downloading). After 30 days, outstanding amounts will be subject to a service charge of $1-1 / 2 \%$ per month or, if less, the maximum rate allowed by applicable law. Unless otherwise specifically set forth in the Order Confirmation or in a separate written agreement signed by CCC, invoices are due and payable on "net 30 " terms. While User may exercise the rights licensed immediately upon issuance of the Order Confirmation, the license is automatically revoked and is null and void, as if it had never been issued, if complete payment for the license is not received on a timely basis either from User directly or through a payment agent, such as a credit card company. 3.3 Unless otherwise provided in the Order Confirmation, any grant of rights to User (i) is "one-time" (including the editions and product family specified in the license), (ii) is non-exclusive and non-transferable and (iii) is subject to any and all limitations and restrictions (such as, but not limited to, limitations on duration of use or circulation) included in the Order Confirmation or invoice and/or in these terms and conditions. 
Upon completion of the licensed use, User shall either secure a new permission for further use of the Work(s) or immediately cease any new use of the Work(s) and shall render inaccessible (such as by deleting or by removing or severing links or other locators) any further copies of the Work (except for copies printed on paper in accordance with this license and still in User's stock at the end of such period). 3.4 In the event that the material for which a republication license is sought includes third party materials (such as photographs, illustrations, graphs, inserts and similar materials) which are identified in such material as having been used by permission, User is responsible for identifying, and seeking separate licenses (under this Service or otherwise) for, any of such third party materials; without a separate license, such third party materials may not be used.

3.5 Use of proper copyright notice for a Work is required as a condition of any license granted under the Service. Unless otherwise provided in the Order Confirmation, a proper copyright notice will read substantially as follows: "Republished with permission of [Rightsholder's name], from [Work's title, author, volume, edition number and year of copyright]; permission conveyed through Copyright Clearance Center, Inc. ” Such notice must be provided in a reasonably legible font size and must be placed either immediately adjacent to the Work as used (for example, as part of a by-line or footnote but not as a separate electronic link) or in the place where substantially all other credits or notices for the new work containing the republished Work are located. Failure to include the required notice results in loss to the Rightsholder and CCC, and the User shall be liable to pay liquidated damages for each such failure equal to twice the use fee specified in the Order Confirmation, in addition to the use fee itself and any other fees and charges specified.

3.6 User may only make alterations to the Work if and as expressly set forth in the Order Confirmation. No Work may be used in any way that is defamatory, violates the rights of third parties (including such third parties' rights of copyright, privacy, publicity, or other tangible or intangible property), or is otherwise illegal, sexually explicit or obscene. In addition, User may not conjoin a Work with any other material that may result in damage to the reputation of the Rightsholder. User agrees to inform CCC if it becomes aware of any infringement of any rights in a Work and to cooperate with any reasonable request of $\mathrm{CCC}$ or the Rightsholder in connection therewith.

4. Indemnity. User hereby indemnifies and agrees to defend the Rightsholder and CCC, and their respective employees and directors, against all claims, liability, damages, costs and expenses, including legal fees and expenses, arising out of any use of a Work beyond the scope of the rights granted herein, or any use of a Work which has been altered in any unauthorized way by User, including claims of defamation or infringement of rights of copyright, publicity, privacy or other tangible or intangible property. 5. Limitation of Liability. UNDER NO CIRCUMSTANCES WILL CCC OR THE RIGHTSHOLDER BE LIABLE FOR ANY DIRECT, INDIRECT, CONSEQUENTIAL OR INCIDENTAL DAMAGES (INCLUDING WITHOUT LIMITATION DAMAGES FOR LOSS OF BUSINESS PROFITS OR INFORMATION, OR FOR BUSINESS INTERRUPTION) ARISING OUT OF THE USE OR INABILITY TO USE A WORK, EVEN IF ONE OF THEM HAS BEEN ADVISED OF THE POSSIBILITY OF SUCH DAMAGES. In any event, the total liability of the Rightsholder and CCC (including 
their respective employees and directors) shall not exceed the total amount actually paid by User for this license. User assumes full liability for the actions and omissions of its principals, employees, agents, affiliates, successors and assigns.

6. Limited Warranties. THE WORK(S) AND RIGHT(S) ARE PROVIDED “AS IS". CCC HAS THE RIGHT TO GRANT TO USER THE RIGHTS GRANTED IN THE ORDER CONFIRMATION DOCUMENT. CCC AND THE RIGHTSHOLDER DISCLAIM ALL OTHER WARRANTIES RELATING TO THE WORK(S) AND RIGHT(S), EITHER EXPRESS OR IMPLIED, INCLUDING WITHOUT LIMITATION IMPLIED WARRANTIES OF MERCHANTABILITY OR FITNESS FOR A PARTICULAR PURPOSE. ADDITIONAL RIGHTS MAY BE REQUIRED TO USE ILLUSTRATIONS, GRAPHS, PHOTOGRAPHS, ABSTRACTS, INSERTS OR OTHER PORTIONS OF THE WORK (AS OPPOSED TO THE ENTIRE WORK) IN A MANNER CONTEMPLATED BY USER; USER UNDERSTANDS AND AGREES THAT NEITHER CCC NOR THE RIGHTSHOLDER MAY HAVE SUCH ADDITIONAL RIGHTS TO GRANT.

7. Effect of Breach. Any failure by User to pay any amount when due, or any use by User of a Work beyond the scope of the license set forth in the Order Confirmation and/or these terms and conditions, shall be a material breach of the license created by the Order Confirmation and these terms and conditions. Any breach not cured within 30 days of written notice thereof shall result in immediate termination of such license without further notice. Any unauthorized (but licensable) use of a Work that is terminated immediately upon notice thereof may be liquidated by payment of the Rightsholder's ordinary license price therefor; any unauthorized (and unlicensable) use that is not terminated immediately for any reason (including, for example, because materials containing the Work cannot reasonably be recalled) will be subject to all remedies available at law or in equity, but in no event to a payment of less than three times the Rightsholder's ordinary license price for the most closely analogous licensable use plus Rightsholder's and/or CCC's costs and expenses incurred in collecting such payment.

\section{Miscellaneous.}

8.1 User acknowledges that CCC may, from time to time, make changes or additions to the Service or to these terms and conditions, and CCC reserves the right to send notice to the User by electronic mail or otherwise for the purposes of notifying User of such changes or additions; provided that any such changes or additions shall not apply to permissions already secured and paid for.

8.2 Use of User-related information collected through the Service is governed by CCC's privacy policy, available online here:http://www.copyright.com/content/cc3/en/tools/footer/privacypolicy.html. 8.3 The licensing transaction described in the Order Confirmation is personal to User. Therefore, User may not assign or transfer to any other person (whether a natural person or an organization of any kind) the license created by the Order Confirmation and these terms and conditions or any rights granted hereunder; provided, however, that User may assign such license in its entirety on written notice to CCC in the event of a transfer of all or substantially all of User's rights in the new material which includes the Work(s) licensed under this Service. 
8.4 No amendment or waiver of any terms is binding unless set forth in writing and signed by the parties. The Rightsholder and CCC hereby object to any terms contained in any writing prepared by the User or its principals, employees, agents or affiliates and purporting to govern or otherwise relate to the licensing transaction described in the Order Confirmation, which terms are in any way inconsistent with any terms set forth in the Order Confirmation and/or in these terms and conditions or CCC's standard operating procedures, whether such writing is prepared prior to, simultaneously with or subsequent to the Order Confirmation, and whether such writing appears on a copy of the Order Confirmation or in a separate instrument.

8.5 The licensing transaction described in the Order Confirmation document shall be governed by and construed under the law of the State of New York, USA, without regard to the principles thereof of conflicts of law. Any case, controversy, suit, action, or proceeding arising out of, in connection with, or related to such licensing transaction shall be brought, at CCC's sole discretion, in any federal or state court located in the County of New York, State of New York, USA, or in any federal or state court whose geographical jurisdiction covers the location of the Rightsholder set forth in the Order Confirmation. The parties expressly submit to the personal jurisdiction and venue of each such federal or state court.If you have any comments or questions about the Service or Copyright Clearance Center, please contact us at 978-750-8400 or send an e-mail to info@copyright.com.

v 1.1

Questions? customercare@copyright.com or +1-855-239-3415 (toll free in the US) or +1-978-646-2777. 